University of Louisville

ThinkIR: The University of Louisville's Institutional Repository

Electronic Theses and Dissertations

8-2014

\title{
The second generation of the CCCM system for in-vitro cardiac tissue engineering.
}

Mai-Dung Thi Nguyen

University of Louisville

Follow this and additional works at: https://ir.library.louisville.edu/etd

Part of the Mechanical Engineering Commons

\section{Recommended Citation}

Nguyen, Mai-Dung Thi, "The second generation of the CCCM system for in-vitro cardiac tissue engineering." (2014). Electronic Theses and Dissertations. Paper 1058.

https://doi.org/10.18297/etd/1058

This Doctoral Dissertation is brought to you for free and open access by ThinkIR: The University of Louisville's Institutional Repository. It has been accepted for inclusion in Electronic Theses and Dissertations by an authorized administrator of ThinkIR: The University of Louisville's Institutional Repository. This title appears here courtesy of the author, who has retained all other copyrights. For more information, please contact thinkir@louisville.edu. 


\title{
THE SECOND GENERATION OF THE CCCM SYSTEM FOR IN-VITRO CARDIAC TISSUE ENGINEERING
}

\author{
By \\ Mai Dung Thi Nguyen \\ B.S., the Oklahoma University, 1999 \\ M.S., the University of Louisville, 2010

\begin{abstract}
A Dissertation
Submitted to the Graduate Faculty of

J.B. Speed School of Engineering, the University of Louisville, in Partial Fulfillment of the Requirements

for the Degree of
\end{abstract}

Doctor of Philosophy

Mechanical Engineering Department

University of Louisville

Louisville, KY

August, 2014 
Copyright 2014 by Mai-Dung Thi Nguyen

All rights reserved 



\title{
THE SECOND GENERATION OF THE CCCM SYSTEM FOR IN-VITRO CARDIAC TISSUE ENGINEERING \\ By
}

\author{
Mai Dung Thi Nguyen \\ B.S., the Oklahoma University, 1999 \\ M.S., the University of Louisville, 2010
}

A Dissertation Approved on

June 17, 2014

By the following Dissertation Committee:

Dr. Guruprasad Giridharan, Director

Dr. Thomas Berfield, Co-chair

Dr. Bradley B. Keller

Dr. Palaniappan Sethu

Dr. Stuart Williams

Dr. Ayman El-Baz 


\section{DEDICATION}

This dissertation is gratefully dedicated

to

my lovely deceased aunt, Say Nguyen,

my biological family,

and the Dominican Sisters of Peace 


\section{ACKNOWLEDGMENTS}

My deepest gratitude goes to Dr. Bradley Keller, Dr. Guruprasad Giridharan, and Dr. Palaniappan Sethu, who welcomed me and provided tremendous opportunities to explore my career with trust, encouragement, consistent advice, and challenges. With their guidance, my project moved far beyond my imagination. I also would like to thank Dr. Thomas Berfield and Dr. Steward Williams, who were so generous with their time to serve as my dissertation committee. I am grateful to their wisdom, ideas, and support.

To various members of the Microscale Biotechnology Lab and Pediatric Cardiology Research lab, especially Dr. Rosendo Estrada, and Mr. Joseph Tinney, Dr. Fei Ye, and Dr. Fangping Yuan, I am grateful. During my PhD study, they gave me much help and created a good working, collaborating atmosphere in the labs.

This project wouldn't be accomplished without the help and encouragement of other professors behind the scene, including Dr. Roger Bradshaw from the mechanical department, Dr. Walsh, Dr. Cindy Harnett, and Dr. Thomas Roussel from the electrical department, Dr. Ayman El-Baz from bioengineering department, as well as my former supervisors Dr. B. Clark and Dr. P. Feldholf from the Molecular and Biochemistry department.

I would like to thank the clean room staff and mechanical shop staff, especially Mr. Jones John for device fabrication and for making device components. 
English is my second language, and writing is always challenging task for me. I deeply appreciate Sister Judy Morris OP, from the Dominican Sisters of Peace, who have been correcting grammar and editing my papers.

Last but not least, I would like to express my sincere gratitude to my biological family, the congregation of the Dominican Sisters of Peace, and friends. I am grateful for all who have been part of my journey, encouraging me to catch the dream of my life, day and night, without fear, and supporting me during these years.

Throughout the course of this $\mathrm{PhD}$ program was supported by the following grants: SREB PhD Fellowship, Graduate School Scholarship, the Wallace H. Coulter Foundation (PI: Dr. Sethu), NIH R15 grant no. 1R15HL115556-01A1 (PI: Dr. G. Giridharan), NSF Grant No. 0814194 (PI: Dr. B. Keller), and the KOSAIR pediatric cardiac research fund (PI: Dr. B. Keller). I would like to acknowledge and thank these funding sources for making this $\mathrm{PhD}$ study and research work possible.) 


\title{
ABSTRACT \\ THE SECOND GENERATION OF CCCM SYSTEM FOR IN-VITRO
}

\section{CARDIAC TISSUE ENGINEERING}

\author{
Mai Dung Thi Nguyen
}

June 17, 2014

Cardiovascular disease is the leading cause of death worldwide. When a myocardial infarction occurs, scar tissue compensates the damaged myocardial tissue. This scar tissue increases the stiffness of the heart tissue, reduces the heart's function, and finally leads to the heart failure (HF) disease. To have the tissue engraftment, in-vitro cardiac tissue should have the same properties as the native mature cardiac tissue. However, current in-vitro cell culture technologies fail to accurately recreate the in-vivo like mechanically physiological environment for in-vitro cardiac tissue culture, and therefore, fail to regenerate the in-vivo like mature cardiac tissue. Hence, a microfluidic cardiac cell culture model (CCCM) system was developed to better recreate the cellular environment and advance cardiac regeneration. CCCM system replicates the hemodynamic loading and unloading conditions occurring inside the left ventricle of a heart. With this system, different pressures of human heart conditions may be replicated for a variety of clinical and physiologic conditions. For proof-of-concept, embryonic chick cardiac cells with normal heart condition were applied. Compared to the tissue cultured in a static condition, tissues stimulated in the CCCM system achieved an in-vivo like cardiac 
matured phenotype, had higher proliferating rate, showed more maturity, and expressed more contractile proteins. These results demonstrated that the CCCM system can be used to study the behavior of cardiomyocytes in different mechanical heart conditions and to create mature cardiac tissue which will benefit cardiac tissue transplant for HF. 


\section{TABLE OF CONTENTS}

PAGE

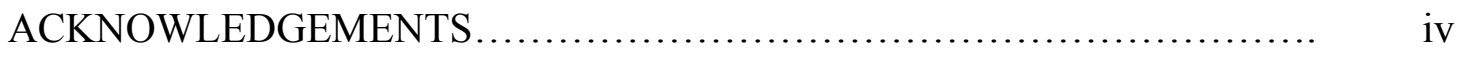

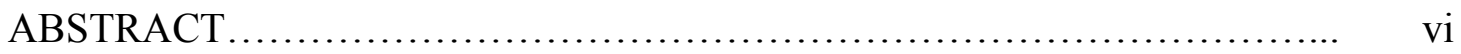

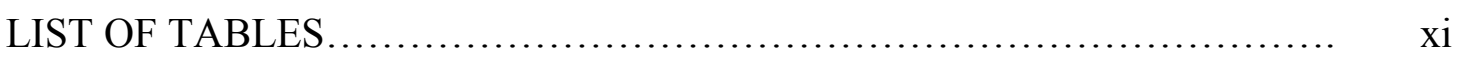

LIST OF FIGURES ................................................ xii

\section{CHAPTERS}

1. INTRODUCTION

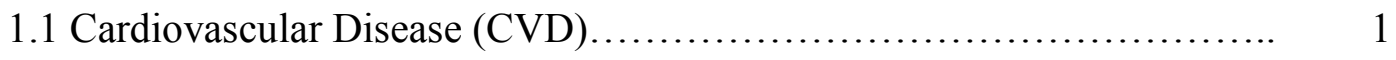

1.2 Treatment of Heart Failure..................................... 3

1.3 The Heart..................................................... 5

1.4 Heart at Cellular level........................................... 10

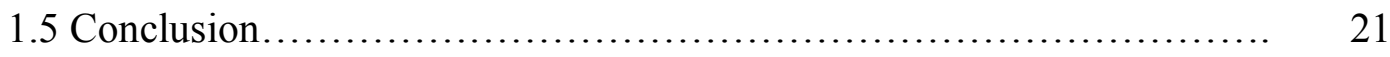

2. IN-VITRO MODEL SYSTEM TO STUDY CARDIAC TISSUE

2.1 Need for Cellular-Level Studies............................... 22

2.2 Important Considerations for Cell-Level Methods of Cardiac Tissue..... $\quad 26$

2.3 Review and Current State of the Art.............................. 27

2.4 Physiologically Relevant Systems for Cardiac Cell Culture............. 29

2.5 In-vitro Techniques for Mechanical Stimulation of the Cells............. 31

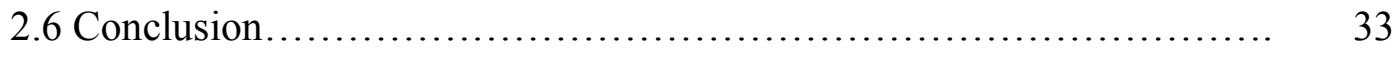




\section{THE $\mu$ CCCM SYSTEM}

3.1 Introduction...................................................... 34

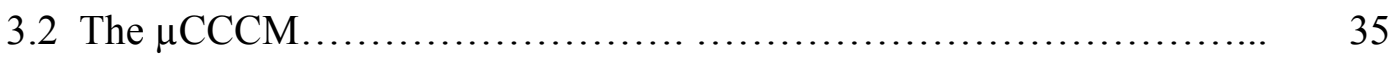

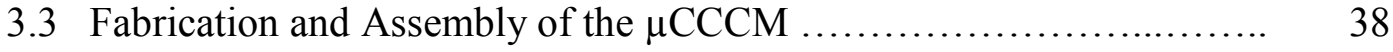

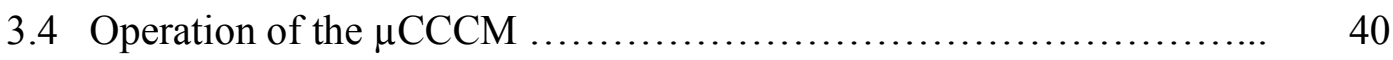

3.5 Applications...................................................... 41

3.6 Conclusion and Limitations ....................................... 46

4. THE SECOND GENERATION CCCM SYSTEM

4.1 Components of the Second Generation CCCM System ................. 49

4.2 Design and Fabrication of Different Components of the CCCM system.. 55

4.3 Assembly of the Whole CCCM system.................................. 63

4.4 Working Principles of the CCCM System............................ 65

4.5 Operation of the CCCM System................................... 67

4.6 Conclusion ...................................................... 68

\section{CHARACTERIZATION OF THE CCCM SYSTEM}

5.1 Young's Modulus of the thin PDMS membrane .......................... 69

5.2 Pressure Generated inside the CCCM Cell Culture Chamber ............. 73

5.3 Strain of the PDMS Cell Culture Membrane..................................... 76

5.4 Shear Stress Modeling............................................. 85

5.5 Conclusion........................................................ 87

6. USING THE CCCM SYSTEM FOR CARDIOMYCYTES STUDIES

6.1 Building the Extracellular Matrix (ECM) for Cardiac Cell Culture........ 89

6.2 Culturing Cardiomyocytes in the CCCM system....................... 91 
6.3 Cardiac Tissue Alignment and Proliferation....................... 98

6.4 Cardiac Tissue Maturation ...................................... 103

6.5 Cardiac Tissue Response to $\beta$-Adrenergic Stimulation............... 108

6.6 Selected Cardiomyocyte Gene Expressions and Protein Synthesis in

Response to Mechanical Conditioning........................... 114

6.7 Conclusion .................................................... 127

7. CONCLUSION

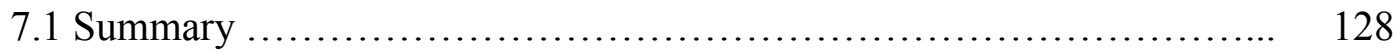

7.2 Limitations................................................ 129

7.3 Future Work. ................................................ 130

REFERENCES..................................................... 132

APPENDIX

List of Abbreviations.........................................

CURRICULUM VITAE................................................ 149 


\section{LIST OF TABLES}

TABLES

PAGE

3.1 Summary of similarities and differences (in italic) between the left ventricle and the $\mu \mathrm{CCCM}$ system................................. 48

4.1 Dimensions of each part of the oil chamber......................... 61

5.1 Hemodynamic parameters corresponding to left ventricular mechanical loading during various clinical conditions were replicated using the CCCM system. 


\section{LIST OF FIGURES}

FIGURES

PAGE

1.1 Heart Failure is the end-stage of dysfunctional cardiovascular system existing for a long term.......................................... 3

1.2 Schematic image of heart gross anatomy.............................. 7

1.3 Relationship between pressure and volume during the pump cycle of the heart............................................................... 10

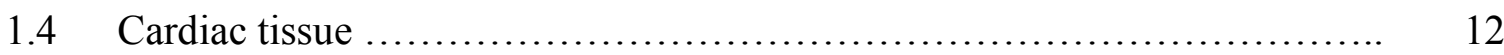

1.5 Simplified schematic model of mature myofibril....................... 14

1.6 Simplified schematic of the biomechanical pathway relating to calcium regulation inside the cell for the contractility.

1.7 Maladaptive responses due to abnormal mechanical stimulus leading to

heart failure...

2.1 Biologic complexity versus clinical translation plot....

3.1 Assembled $\mu \mathrm{CCCM}$ for cell culture experiment........................ 36

3.2 Setup design for equi-baxial strain.....................................

3.3 Schematic representation of the top and bottom components of the platform for housing the cell culture chamber.

3.4 Assembly of the cell culture and oil chambers between the platforms. 
3.5 Pressure waveforms that simulate various physiologic conditions in the

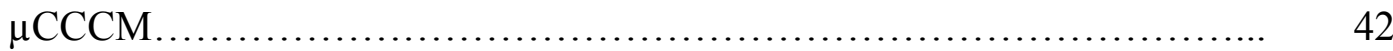

3.6 Physiological loading promoted the alignment of f-actin and increased phosphorylated phospholamban activity in H9C2 cells................... 45

4.1 A schematic image of the second generation CCCM system............... 50

4.2 Scheme of the CCCM cell culture platform station........................ 52

4.3 Scheme of the PDMS membrane deflection under the influence of fluid and pneumatic pressure loading and unloading.

4.4 Image of the CCCM system connected to the LabView control system via pressure sensor.

4.5 Schematic of the top and bottom plates of the station for housing the cell culture chamber

4.6 Graph of PDMS membrane thickness versus spinning speed

4.7 Fabrication steps of making a thin PDMS membrane via the gasket method..

4.8 Fabrication steps involved in construction of the cell chamber and the medium reservoir

4.9 Schematic representation of fabrication steps involved in construction of the assessable pressure chamber.

4.10 Schematic image of the oil chamber and the post membrane....

4.11 Schematic representation of fabrication steps involved in construction of the post membrane.

4.12 Schematic of an assembled CCCM platform.

4.13 Picture of the CCCM experimental setup 
4.14 A working cycle of the CCCM system and the pressure-volume loop occurred in left ventricle of the heart............................... 66

5.1 Experimental setup image for strain and Young's Modulus measurement of PDMS membranes................................................. $\quad 71$

5.2 Graphs of stress versus strain of thin PDMS sheets ..................... 73

5.3 Pressure vs time plots representing the changes in pressure within the CCCM cell culture chamber for different human heart conditions............ 75

5.4 Scheme for the stretches of the circular membrane....................... 76

5.5 Schematic demonstration for radial strain and circumferential strain measurements for a thin PDMS circular membrane...................... 79

5.6 Pictures of the PDMS membrane deflections via the ANSYS ................ 79

5.7 Plots of displacement and strain of the circular PDMS membrane............ 80

5.8 Images of experimental set up for making movies of the PDMS membrane deflections using the Ultra Sound system............................. 81

5.9 Ultrasound images of PDMS membrane deflection at 0,25 , and $50 \mu 1$ fluid loading along with the real set-up experiement........................ 82

5.10 Results of the PDMS membrane deflection............................ 84

5.11 Flow velocity simulation for the cell culture chamber of the $\mu$ CCCM........ 87

6.1 Image of the collagen matrix with cardiac cells after 4 day cultured in the

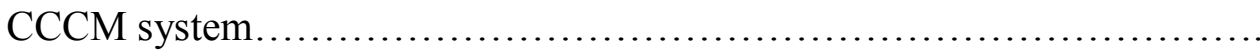

6.2 Plot of pressure vs time and pressure-volume loop obtained within the

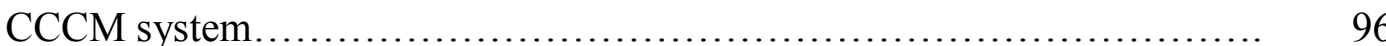

6.3 Phase contrast images of in vitro embryonic chick cardiac cells cultured 
under the static and CCCM stimulated conditions.....

6.4 Immunofluorescence images of cardiomyocyte tissue cultured in the CCCM system and in the static condition.

6.5 Results from immunofluorescence staining for cell proliferation.....

6.6 Graph of the beat rate (bpm) between the control and the stimulated groups.

6.7 Electrical Pacing Setup System.

6.8 Pacing Electrical Stimulation Results.

6.9 Graph of cardiomyocyte shortening with and without electrical stimulation.

6.10 Plot represents the percentage change in beat rate of the cardiac tissue under the effects of Isoproterenol

6.11 Changes in the cardiomyocyte contractions with and without the influence of ISO.

6.12 Plots of fold changes in gene expression with ventricular gene expression set as the control state.

6.13 Graph of fold change in gene expressions with ventricular gene expression set as the control state.

6.14 Total RNA obtained from 2D embryonic chick cardiac tissue.

6.15 Total proteins of the 2D cardiac tissue constructs

6.16 Images of Western Blot bands of the interested proteins.

6.17 Graphs of SERCA2a, $\alpha$ - and $\beta$-MHC proteins normalized by $\beta$-Actin.

6.18 Graphs of TnT and $\alpha$-Actinin proteins normalized by $\beta$-Actin 


\section{CHAPTER 1 INTRODUCTION}

\subsection{CARDIOVASCULAR DISEASE (CVD)}

The basic components of the cardiovascular system are the heart, blood vessels (arteries, capillaries, and veins), autonomic innervation of the heart and vasculature, and the circulating blood. The heart acts as a pump to circulate blood through the lungs to oxygenate, and through the arteries to deliver oxygen and nutrients to the entire body. When blood is delivered to tissues, the oxygen and carbon dioxide are exchanged. The deoxygenated blood is then returned to the heart via veins, and finally to the lungs, helping dispose of carbon dioxide and other waste products.

Cardiovascular Disease (CVD) collectively refers to abnormalities associated with various components of the cardiovascular system and covers over 60 disorders relating to the heart and vascular system. CVD is the leading cause of death in the United States, which is higher than the next four leading causes of death combined. ${ }^{1}$ Statistics from the American Heart Associate (AHA) indicate that about 81 million people or $1 / 4^{\text {th }}$ of the population suffer from some form of CVD in the United States. This includes high blood pressure $\sim 74.5$ million; coronary heart disease $\sim 17.6$ million; stroke $\sim 6.4$ million; congenital cardiovascular defects $\sim 650,000$ to 1.3 million; and heart failure $\sim 5.8$ million. ${ }^{2} \quad$ Based on the article: "Heart Disease and Stroke 2010 Update" from AHA, it 
was estimated that the total indirect and direct cost due to CVD and stroke in 2010 would be $\sim 503.2$ billion dollars, placing a severe burden on the economy and diminishing the quality of life for several thousands of people..$^{2-4}$

CVD can be classified into two major categories based on disorders of the heart or the vascular system. Disorders of the heart include conditions such as angina pectoris, arrhythmias, cardiomyopathy, congestive heart failure, and congenital heart disease. Disorders of the vascular system, on the other hand, include conditions such as aortic aneurysms, arteriosclerosis, high blood pressure, stroke, and transient ischemia. ${ }^{1,5}$ Coronary heart disease is the most common type of CVD. It is a condition which fatty deposits build up inside the inner walls of coronary arteries, creating blockages of the arteries. These blockages reduce the blood flow, causing a pain or discomfort in the chest, heart failure (HF) and heart attacks. In atherosclerosis, the arteries become thicker or harden; and the inner wall of the arteries becomes narrower due to the deposits of fat, cholesterol or other substances. This narrowed passage can block the blood flow and lead to heart attacks and strokes. Strokes occur when the blood vessels to the brain are occluded or broken. As a result, brain cells begin to die due to lack of oxygen and nutrition. ${ }^{2-4}$ Stroke usually relates closely to high blood pressure, which happens when the pressure inside the vessels is higher than the normal range, which is about $120 / 80$ mmHg. High blood pressure can cause HF, stroke, kidney failure, or other health problems. The end stage for different CVDs is HF (Fig.1.1) which occurs when the heart fails to pump sufficient blood through the body. The body, therefore, does not get enough oxygen and nutrients. HF may develop slowly over time, and if not treated, can 
cause life-threatening conditions, including pulmonary edema, failure of brain, kidney and other major organs, and myocardial infarction. ${ }^{6-9}$

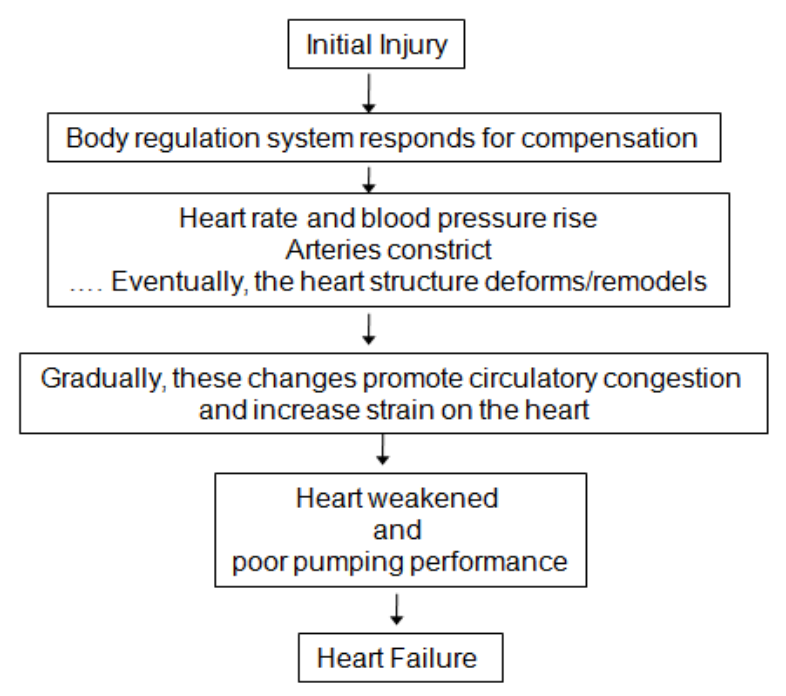

Figure 1.1: Heart Failure is the end-stage of dysfunctional cardiovascular system existing for a long term.

\subsection{TREATMENT OF HEART FAILURE}

Heart Failure (HF) is a chronic disease, therefore, the treatment for HF needs lifelong management, which includes changes in living habits, medication, and assist devices to augment heart function. ${ }^{7,10,11}$ The only cure for end- stage heart failure is total heart transplantation.

Drug therapy is usually used for the initial stage of the HF. Depending on the stage and symptoms of HF, a single or combination of different medications are applied. The most common drugs used for treatment of HF include Angiotensin-converting enzyme inhibitors, or Angiotensin II receptor blockers, Digoxin (Lanoxin), Beta blockers, Diuretics, and Aldosterone antagonists. ${ }^{12}$ Angiotensin-converting enzyme inhibitors or 
Angiotensin II receptor blockers dilate the arteries and improve blood flow, therefore, decrease the workload on the heart. Digoxin helps to strengthen the contraction of heart muscle and slow the heartbeat. Beta blockers reduce the heart rate and pressure. Diuretics enhance urination frequency to keep from the fluid building up in the body, especially in the lungs. Aldosterone antagonists help the heart work better.

When the HF advances to a more serious stage, devices that optimize myocardial function may be used for the treatment. For example, implantable cardioverterdefibrillators are used for monitoring the heart rhythm. Cardiac resynchronization therapy, or biventricular pacing, are used to help the heart pump efficiently by sending timed electrical impulses to the heart's ventricles for contraction. Mechanical circulatory support devices (left ventricular assist devices (LVAD) ) that unload the native ventricle are used as a bridge to transplantation or as destination therapy in patients with severe HF that are refractory to medical therapy. ${ }^{13}$ LVADs are mechanical pumps designed to transport blood from the left ventricle through the body.

Heart transplant is a palliative therapy used for a subset of pediatric and adult patients with progressive and irreversible HF. A healthier heart from a donor is replaced for the failing diseased heart. ${ }^{14}$ However, people undergoing heart transplant face high risks of the rejection of the donor heart, infection, artery problems, medication side effects and the potential high risk of cancer due to the immune-suppressant medications. ${ }^{14}$ In addition, due to the shortage of donors, people who need heart transplants experience a long waiting times. Heart transplant or LVAD are currently the only options for the end- 
stage HF. However, due to the lack of donors and the complications associated with immunosuppressive treatments as well as many high risks occurred during surgery, scientists and surgeons continue to search for new strategies to treat the HF.

\subsection{THE HEART}

\subsubsection{Overview of the formation of the heart in the embryo}

The heart is one of the first functioning organs formed during the embryonic period, both in bird and mammalian embryos. ${ }^{15,16}$ The heart derives from the anterior mesoderm, the middle embryonic layer. First, one group of the mesodermal cells differentiate into precardiac cells, which later transform into myocardial, endothelial and smooth muscle cells. These mesodermal precardiac cells migrate and form the primitive linear heart tube, which includes the inner endothelial, cardiac jelly, and external myocardial layers. Another population of cardiac cells differentiates into cardiac myocytes, smooth muscle cells, and endothelial cells. ${ }^{17,} 18$ Then, the primitive straight heart tube loops and folds into an S-shape, followed by the convergence process. During this convergence phase, the formation of trabeculae begins; and hemodynamic stimulation plays an important role in modulating the trabecular patterning and development. ${ }^{19}$ The heart surface area, myocardial mass and wall stiffness increase as a result of this trabecular formation. At the end of this formation, the trabecular compaction begins, which contributes to the formation of ventricular septum and the thickness of the ventricular compact layer. At this time, the separation between the atria and ventricles, via the inlet segment, occurs and the formation of atria and ventricle develops. ${ }^{17,20}$ Following the convergence is the wedging process. The modification of valves, such as the aortic valve or atria-ventricular valves, occurs; and the separation of left and right atria and ventricles is completed to 
form a regular 4-chamber heart. ${ }^{15,19,20,19}$ Any abnormality during these processes can lead to heart congenital heart defects.

A complete 4-chamber heart, through one organ, functions as two pumps coupled to one another in a series. The right pump receives deoxygenated blood from the entire body and pumps it to the lungs for oxygen-carbon dioxide exchange, while the left pump receives the oxygenated blood from the lung and then delivers this oxygenated blood to the entire body. As a result, the pressure in the right pump is lower than in the left pump. However, to perform pumping function, both the left and right pumps need to match their output volume.

\subsubsection{Gross anatomy of the heart}

Each side of a heart has a main pumping chamber (the ventricle), a holding chamber (the atria), and two valves to prevent back flow (Fig. 1.2.). The valve between the right atria and right ventricle is the tricuspid valve and between the left atria and left ventricle is the mitral valve. ${ }^{21,22}$ These valves prevent the retrograde flow of blood back into the atrium when the ventricles contract. The pulmonic valve and aortic valve in the right and left ventricle respectively prevent the blood in the pulmonary artery and aorta from flowing back to the right and left ventricle when these ventricles relax. The two ventricles share a common wall (septum) and electrical system to stimulate and control the timing of native ventricular contraction. 


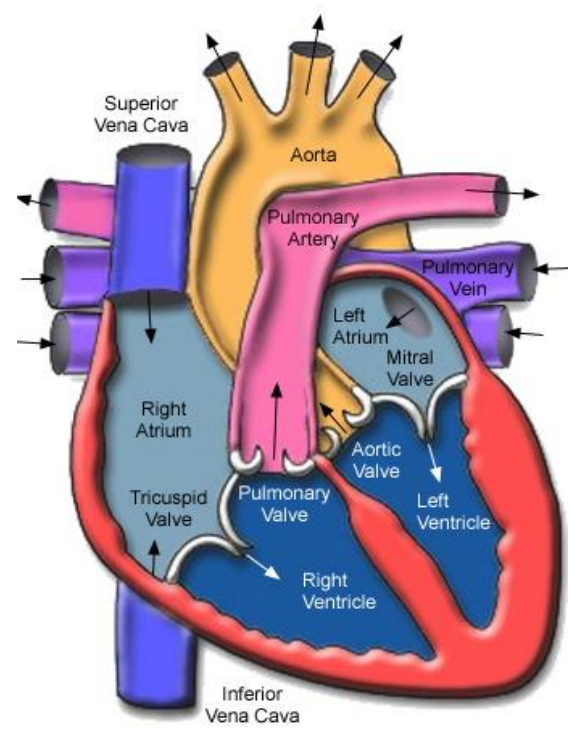

Figure 1.2: Schematic image of the heart gross anatomy.

(http://healthinformation.4arabs.com/heart/2.html)

Deoxygenated blood from the vena cava system flows to the heart and is held in the right atria. When the tricuspid valve opens, the blood flows into the right ventricle in order to be pumped to the lung where the exchange of carbonate-oxygen in the blood occurs. Through the pulmonary artery, the oxygenated blood is then brought to the left atria and finally is pumped into the left ventricle when the mitral valve opens. The contraction of the left ventricle pumps the blood into the aorta from where blood is delivered throughout the body. Due to the vascular resistances and pressures being higher in the systemic circulation than the pulmonary circulation, the left ventricle generates more force to pump. Therefore, the muscle wall of the left ventricle is thicker than the right ventricle. Atria act as a booster pump and hence the walls of the atria are also much thinner than the ventricles. $^{21,22}$ 


\subsubsection{Working of the heart}

The heart, consists of two atria and two ventricles, is the organ responsible for pumping blood throughout the body. The electrical signals generated in the sino atrial node travel through a signal transduction pathway to stimulate the heart tissue. In response to this, the upper then lower chambers of the heart contract sequentially, first both atria, then the ventricles. The working principle of the right heart side is similar to the left heart.

A pumping cycle of the heart can also be divided into two main periods: the diastolic and systolic periods. The diastolic period starts when the aortic valve closes and the muscles begin to relax. The diastolic period ends when the mitral valve closes after filling the blood from the atrium to the ventricle. The systolic period then starts when the mitral valve closes and the muscle begins to contract. This period ends when the aortic valve closes after the blood in the ventricle is ejected to the aorta. ${ }^{21-23}$

The relationship between pressure and volume, especially for the left ventricle (LV) chamber, can be divided into four phases: the ventricular filling or diastole, isovolumic contraction, ejection, and isovolumic relaxation (Fig.1.3.). ${ }^{21-24}$ During the ventricular filling phase, the relaxation of the heart muscles reaches the maximum level. Therefore, when the blood from the atria continues to fill in the ventricle, the pressure of the ventricle goes up gradually and the blood volume is increased significantly, which is represented by phase ' $a$ ' on the pressure-volume loop. When the ventricle pressure reaches a certain level, the muscles contract. ${ }^{24}$ The pressure inside the ventricle at this point is higher than the atria. As a result, the mitral valve closes, but this pressure is not 
sufficient to open the aortic valve. Because all valves are closed, the pressure is increased dramatically while the volume remains the same. This phase is called the isovolumic contraction phase (phase b). At this point, the ventricle begins to contract until the pressure inside the ventricle chamber exceeds the aortic pressure; then the aortic valve opens. The blood inside the left ventricle is then ejected to the aorta, causing a decrease in volume (phase c). However, since the ventricle is still contracting, the pressure inside the ventricle keeps increasing until it reaches its peak, then, decreases when the ventricle begins to relax. The ventricular pressure begins to drop until the pressure in the aorta finally becomes larger than in the ventricle, causing the closure of the aorta valve. The relaxation of the ventricle plus the closure of valves greatly reduces the pressure inside the ventricle while the volume remains unchanged with no blood going in or out of the ventricle. This phase (phase d) generates a vertical line (isovolumic relaxation phase) on the diagram B (Fig.1.3.). When the pressure inside the LV is lower than the left atria pressure, the mitral valve opens and the LV is again filled with blood. The next cardiac cycle begins. ${ }^{21-24}$

During normal adult conditions, the human heart rate is about $70 \pm 10$ beats per minute (bpm). The stroke volume is about $60 \pm 10 \mathrm{~mL} /$ beat and the left ventricular systolic peak pressure approximates of $120 \pm 10 \mathrm{mmHg}$. The left ventricular end diastolic pressure ranges from 5-12 mmHg. ${ }^{25,26}$. Stroke volume is the volume of blood ejected for each beat. Heart rates vary during fetal development and are then higher in the neonatal and adult heart. 

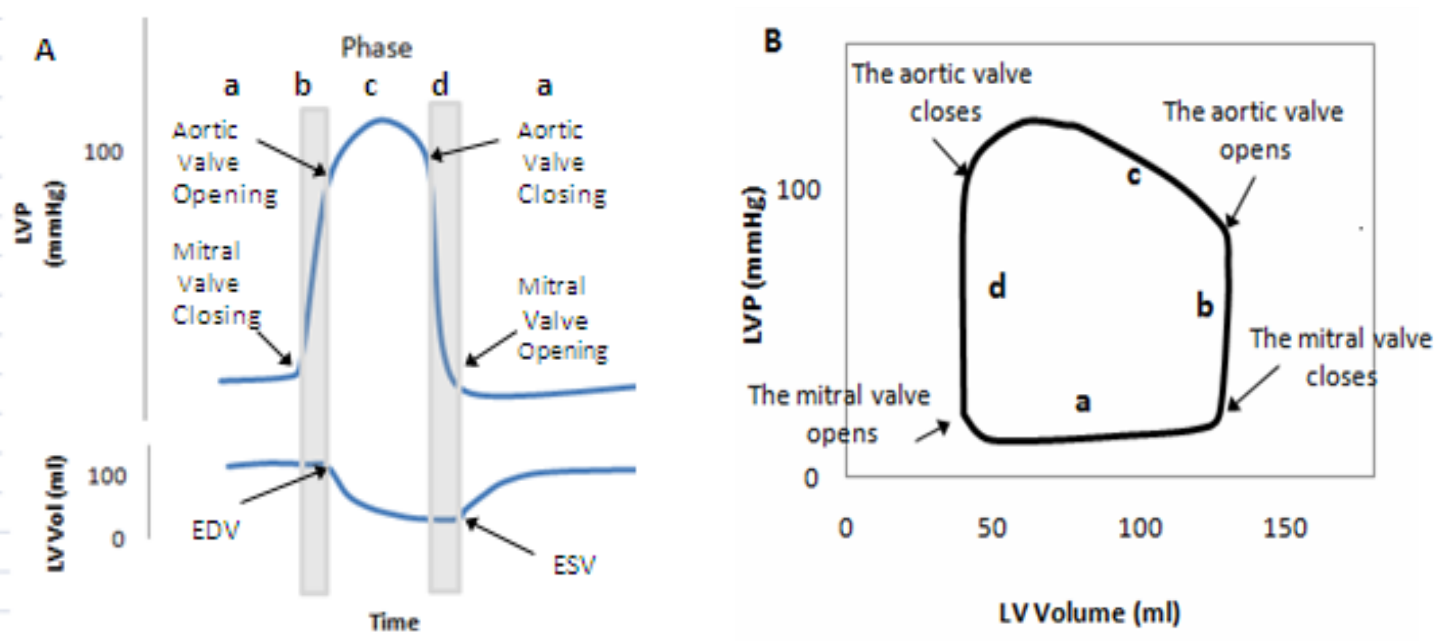

Figure 1.3: Relationship between pressure and volume during the pump cycle of the heart. (A) Cardiac cycle diagram and (B) Pressure-Volume Loop. (a) Diastolic filling phase, (b) Isovolumic phase, (c) ejecting phase, and (d) Isovolumic relaxation phase.

\subsection{HEART AT CELLULAR LEVEL}

\subsubsection{Cellular organization of cardiac tissue}

Unlike other tissue, in the early development, embryonic cardiac tissue has a high ability for both differentiation and proliferation. ${ }^{27-29}$ This high proliferation and differentiation makes the vertebrate heart quickly transformed from a single two-layer straight tube into a four-chamber heart. ${ }^{30}$ Cardiac morphogenesis occurs during the embryonic period, between weeks 4 to 8 in human pregnancy, or between days 2-10 in the chick embryo. ${ }^{16}$ DNA synthesis reaches the high rate during this cardiac morphogenesis; and the cardiac conduction system as well as coronary vascular system develops in late morphogenesis. ${ }^{16 \text {, }}$ ${ }^{31}$ However, when entering the neonatal period, cardiomyoctes reduce their proliferating rate to focus more on producing contractile proteins; hence, becoming functional cardiacmoyctes. ${ }^{32}$ Therefore, cardiomyocyte differentiation occurs to adapt the 
subsequent of hemodynamic pressure/volume loading and unloading, resulting in an increase in size or causing cell hypertrophy. During this hypertrophic process, the amount of protein contents (especially contractile proteins) per individual cell increases with a little change in cell number. ${ }^{33,34}$ After birth, cardiomyocytes lose their ability to proliferate.

The embryonic cardium is composed of three different major cell layers: an inner single cell endocardial layer, a loose connective tissue layer and the layer of cardiomyocyte muscle cells (or myocardium layer). ${ }^{14,}$ 21, 22, 35 During the morphogenesis, via proliferation and differentiation of cardiomyocytes, these three layers are quickly transformed into a 3D trabecular myocardium. (Fig. 1.4.A) This trabecular and compact myocardium continues to increase in mass and number in order to form a solid mature myocardium to sustain the transmural pressure due to the hemodynamic loading and unloading applied on the inner side of the cardiac muscle wall. ${ }^{16}$ Hence, the developing of this solid mature myocardium promotes the myocardium function. ${ }^{36,37}$ To perform this function, under the effect of mechanical load, mature cardiomyocyte and myofibril arrange in parallel. ${ }^{35}$

Cardiomyocytes are the cells that make up the heart muscle and have the ability to contract spontaneously. To perform their function in synchrony, cardiomyocytes need to align and establish end to end cell-cell contacts. (Fig.1.4B and C). Smooth muscle cells form a contiguous layer over the cardiomyocytes, and finally a layer of endothelial cells line the myocardium providing shear protection. Besides these major cell types, cardiac 
tissues are also composed of cardiac fibroblasts. Cardiac fibroblasts relate to heart remodeling, therefore, play a critical role in heart hypertrophy and heart failure. ${ }^{38}$

[A]

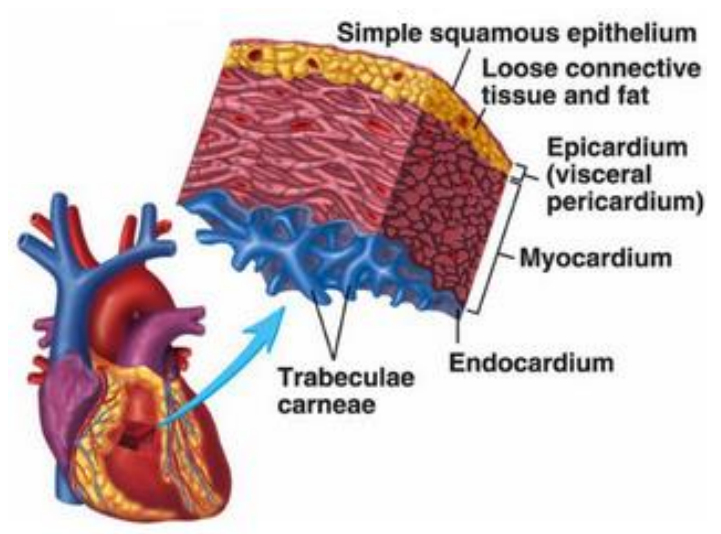

[B]

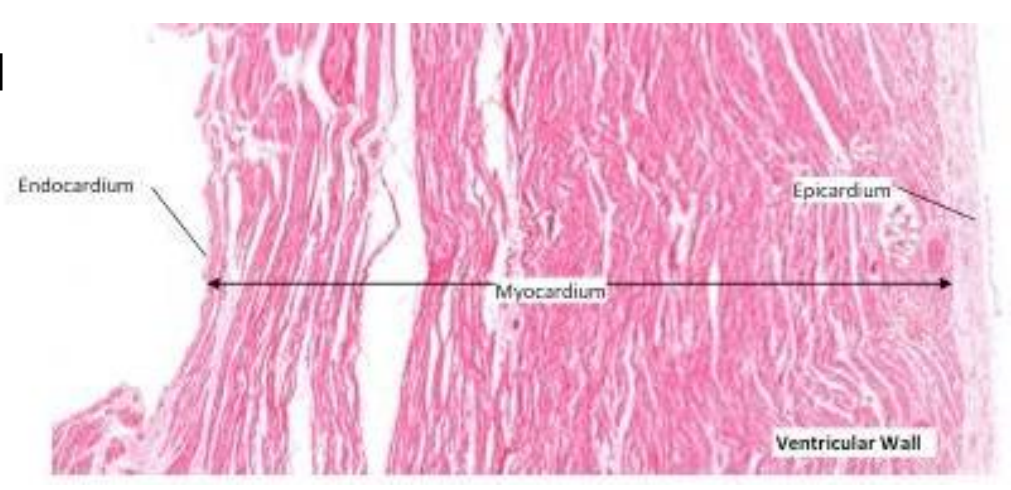

$[\mathrm{C}]$

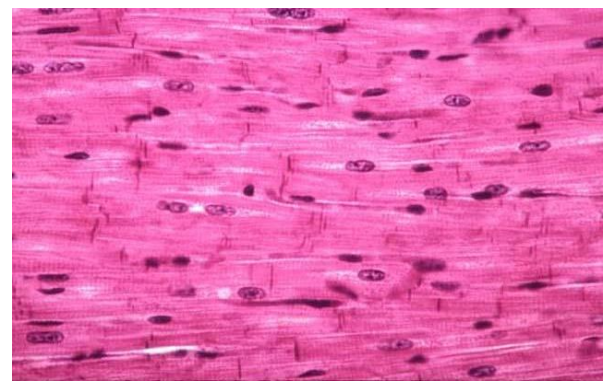

Figure 1.4: Cardiac tissue. [A] Schematic layers of the heart walls; [B] Histological image of ventricular wall; [C] Arrangement of cardiomyocytes in the cardiac muscle. $\left([A]^{39} ;[B]\right.$ http://www.studyblue.com/notes/note/n/histology-medi-1000/deck/938131; [C]:http://www.studyblue.com/notes/note/n/tissue-lab-3-muscle-and-nervoustissue/deck/2179334) 
Hemodynamic stimulation is one of the major exogenous factors regulating cardiac morphogenesis. To adapt mechanical loading and unloading, the myocardium increases the cell size with protein contents, especially the contractile regulating proteins. ${ }^{16,40}$ Myofibril is the contractile structure of cardiac muscle. Myofibrils are composed of sarcomeres or the contractile unit. ${ }^{41,42}$ At the beginning, when the cardiac muscle cells differentiate, all the sarcomeric proteins randomly diffuse in the cytoplasm. By the time of linear-heart-tube formation, due to mechanical stimulations, myocytes begin to beat or contract, causing myofibrils to rapidly arrange into a highly organized network in order to synchronize the contractions throughout. A sarcomere unit is composed of 3 major components: thin filament, thick filament, and $\mathrm{Z}$ band. (Fig. 1.5) Thick filaments arrange in parallel to the thin filaments. Myosin heavy chain proteins are on the thick filament and bind to F-actin of the thin filament during the contraction. Thin filament is composed of F-actin, troponins, and tropomyosin. During the contraction, thick and thin filament slide along each other. Titin filament connects the thick filaments to the $\mathrm{Z}$ band and acts as an elastic spring during the myocyte contraction. The $\mathrm{Z}$ band connects the sarcomere units in series. ${ }^{42-44}$ The contraction is controlled by the interaction between the myosin heavy chain on the thick filament and the binding site of the F-actin molecules, which is the major component of the thin filament. This binding activity depends on the sensitivities of $\mathrm{Ca}^{2+}$ to the tropomyosin-troponin complex. ${ }^{45}$ Alpha-actinin, a component of $\mathrm{Z}$ band, serve as the anchor for $\mathrm{F}$-actin to $\mathrm{Z}$ band, as well as the connection to neighbor sarcomere. ${ }^{46}$ The mechanism of the contraction will be discussed in the section 1.4.2. 
[A]
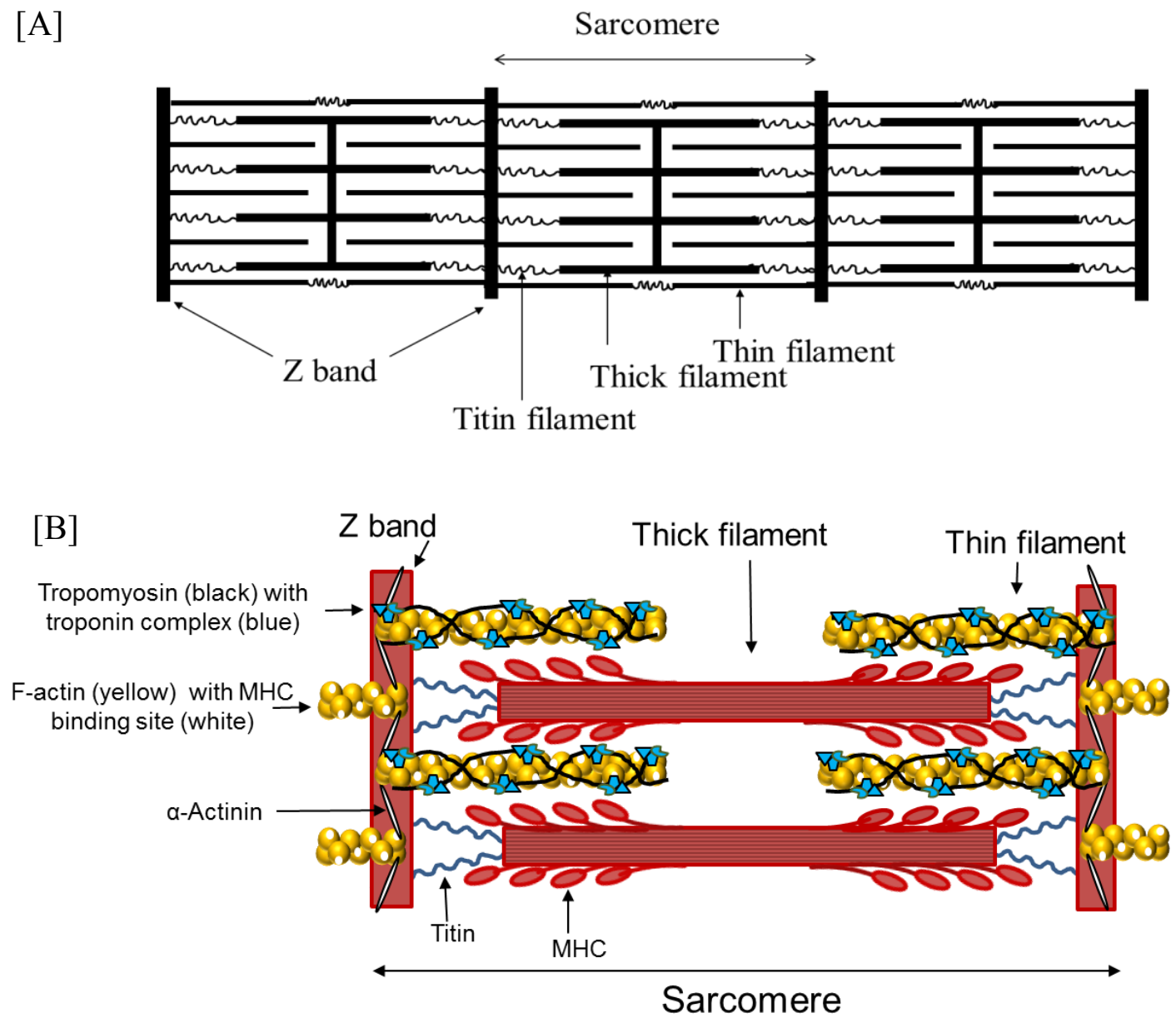

[C]

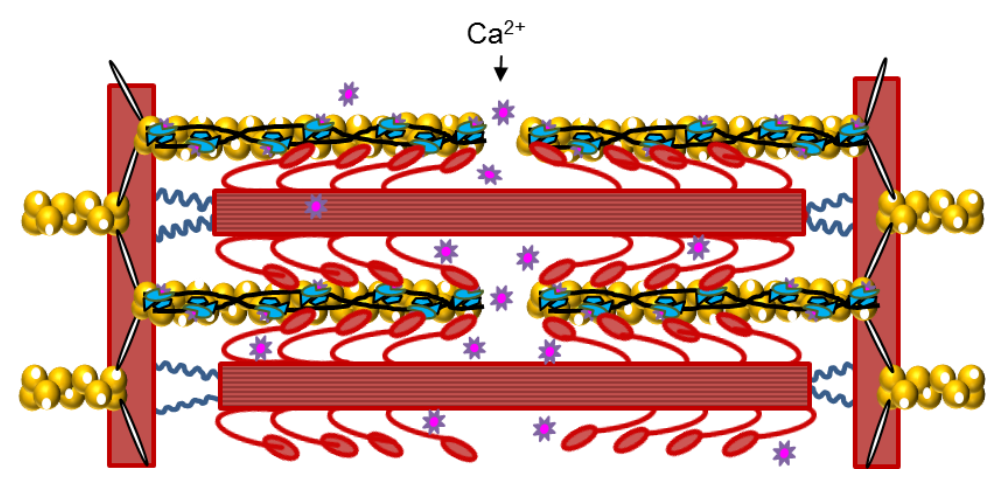

Figure 1.5: Simplified schematic model of mature myofibril. [A] Myofibril; [B] Ultrastructure of a relaxing sarcomere; [C] Ultrastructure of a contracting sarcomere.

The magnitude and duration of stretch and contraction in a cardiac tissue depends on the preload and afterload conditions. In the heart, specifically in the left ventricle, preload 
refers to the condition when the filling of the blood inside the ventricle causes the stretch of the ventricular wall during the diastolic phase. Afterload refers to the aortic resistance that the system needs to overcome in order to pump blood out of the ventricle via opening of the aortic valve. ${ }^{22}$ This directly affects the contractile force that needs to be generated by cardiomyocytes. Overcoming the aortic pressure opens the aortic valve for ejecting the blood into the aorta.

Preload pressure increases slowly when blood filling to the chamber during diastole increases, which creates greater systolic pressure for the subsequent contractions due to the preload sensitivity of the native ventricle. This systolic pressure continues to increase until its maximum systolic pressure is reached at the optimal preload. At a constant preload, higher systolic pressure can be obtained through ventricular contraction by raising the afterload condition such as increasing the aortic pressure. ${ }^{22}$ However, if the afterload pressure or the resistance keeps increasing, the ventricle cannot create enough force to open the aortic valve to push the blood out of the heart. At this point, the systolic phase is totally isovolumic. Preload and afterload pressures, as a result, depend on the vascular system and the behavior of the heart including peripheral resistance, the frequency of the heart's contraction, and change of the stroke volume. ${ }^{22}$

\subsubsection{Cellular response to mechanical signals}

When a mechanical stimulus is applied to the extracellular matrix (ECM), the cells detect this mechanical stimulus via mechanoreceptors. These mechanoreceptors are located in the plasma membrane connecting the extracellular and intracellular regions of the cells, 
traducing the mechanical signals from the outside to the inside of the cell. ${ }^{23,47}$ Once the signal is transmitted inside of the cell, the signal triggers biochemical changes via signaling transduction pathway, enhances the formation of sarcomere, and alters structure of the cytoskeleton directly. ${ }^{23,47}$

Troponin complex includes troponin $\mathrm{C}(\mathrm{TnC})$, troponin $\mathrm{I}(\mathrm{TnI})$, and troponin $\mathrm{T}(\mathrm{TnT})$. TnC is $\mathrm{Ca}^{2+}$ sensitive and has a site for $\mathrm{Ca}^{2+}$ binding; $\mathrm{TnI}$ is the contractile inhibitor; and $\mathrm{TnT}$ is the protein binding to tropomyosin. This troponin complex is strongly associated with the tropomyosin, which regulate the binding of $\mathrm{MHC}$ to the thin filament for sarcomeric contraction. ${ }^{48-50}$ Binding $\mathrm{Ca}^{2+}$ to $\mathrm{TnC}$ switches the conformation of $\mathrm{TnC}$ from a close position to the open one, allowing the interaction of $\mathrm{TnC}$ with $\mathrm{TnI}$ to happen. Without this interaction, TnI binds to F-actin at the binding site of MHC; thus, it prevents the bonding between $\mathrm{MHC}$ with F-actin. Binding of TnC-TnI releases the binding of TnI-actin and enhances the binding of TnI-TnT, which reveals the binding sites of MHC for MHC attachment. Once MHC attaches to the F-actin, with the ATP hydrolysis occurring during the association of $\mathrm{Ca}^{2+}$ with $\mathrm{TnC}$; contraction takes place. ${ }^{41}$, 50, 51 The relaxation of the sarcomere unit occurs during the dissociation of $\mathrm{Ca}^{2+-} \mathrm{TnC}$. $\mathrm{Ca}^{2+}$ is then transferred back to SR via the SERCA2a pump. In summary, calcium ion will bind to the troponin $\mathrm{C}$, help to deactivate troponin I (inhibitor) and change the formation of $\mathrm{TnT}$ in order to reveal the $\mathrm{MHC}$ binding site on the thin filament. Once the binding site is opened, myosin heavy chain from the thick filament binds to F-actin. This binding pulls the thin filament of a sarcomere closer to the center, causing the contraction. Titin is a protein connecting the thick filament to the $\mathrm{Z}$ band at this point 
serves as an elastic spring, keeping the thick filament at the center during this contraction. Alpha actinin, a protein located on the $\mathrm{Z}$ bands, helps to connect the thin filament with the sarcomere, as well as to the thin filament of the neighbor sarcomere. Alpha actinin thus contributes to form and maintain the lattice networks among the sarcomere during the relaxing as well as the contracting phases.

Catecholamine affects mechanical performance of the heart tissues through the activation of $\beta$-adrenergic receptors (AR) and cardiovascular function can be positively and negatively controlled primarily via adrenergic signaling. ${ }^{52-54}$ Under normal physiological conditions, catecholamine induces positive inotropic and lusitropic responses in the heart tissue through $\beta 1$-AR-activated pathway. Once the stimulation of $\beta 1$-ARs binding with its agonist (ex. isoproterenol) happened, this binding complex activates the formation of cyclic AMP (cAMP). cAMP accumulation rapidly increases which indirectly promotes the phosphorylation of PLB, and enhances the cardiac contractility. ${ }^{47}$ Even though the density of $\beta$-ARs in utero diminishes during development, ${ }^{55}$ there are shifts in the adrenergic receptor subtypes with increasing dominance by subtype $\beta 1$-ARs as cardiomyocytes become more mature under normal physiological condition. ${ }^{53,56}$ This dominant $\beta 1$-ARs, therefore, induces positive inotrope and lusitrope via heart rate and contractility. Under the abnormal physiological condition of the heart, or failing heart, $\beta 1$-ARs decreases, and $\beta 2-A R s$ increases with increasing sensitivity to adrenergic toxicity resulting cardiomyocyte death. ${ }^{56}$ 
Increasing cAMP promotes the phosphorylation of phospholamban (PLB). ${ }^{57}$ Sarcoplasmic reticulum calcium ATPase2a (SERCA 2a) and PLB are proteins for calcium handling regulation inside the cardiomyocytes (Fig.1.6). These proteins are involved in regulating the contraction-relaxation of the heart muscle. Phosphorylated PLB enhances the activity of SERCA2a by increasing the affinity of $\mathrm{Ca}^{2+}$ on SERCA2a. This activated SERCA2a then transports calcium against the gradient from cytoplasm

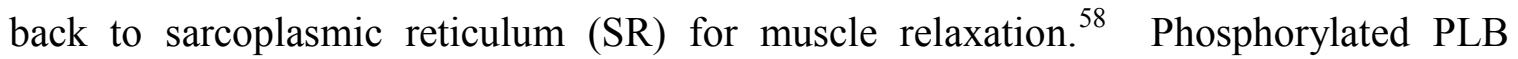
(pPLB) releases this inhibitory effect, making the affinity site to $\mathrm{Ca}^{2+}$ on SERCA2a more apparent. As a result, the rate of calcium ions influx back to the SR increases, promoting the muscle relaxation and being ready for the next contraction. Without being phosphorylated, PLB binds to SERCA2a, preventing the Ca2+ binding to SERCA2a, and hence becoming an endogenous inhibitor of SERCA2a.

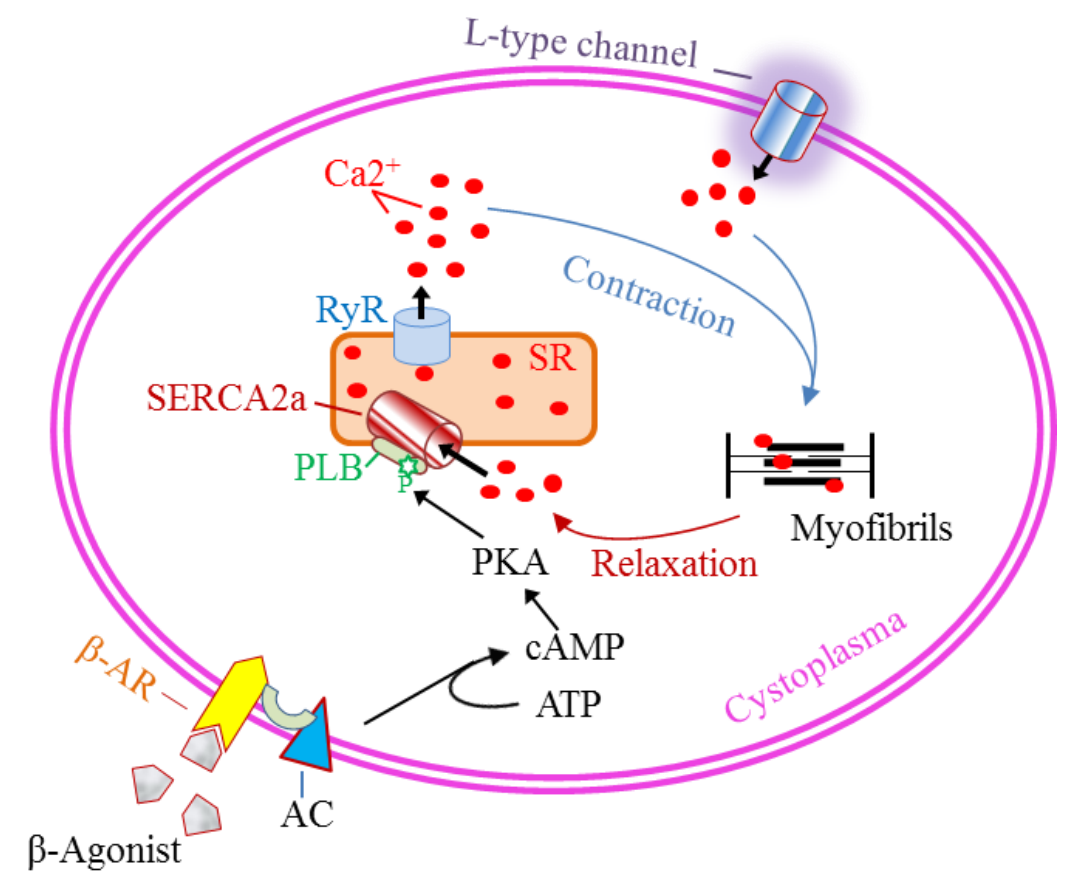

Figure 1.6: Simplified schematic of the biomechanical pathway relating to calcium regulation inside the cell for cardiac contractility. 
Sarcomere length, a basic contractile unit of cardiomyocyte, can be affected by the mechanical stimulation. ${ }^{59}$ When the muscle is stretched to a length greater than normal size, new sarcomere units are added rapidly at the end of the muscle fibers. ${ }^{60,61}$ Unlike the skeletal muscle, small changes in sarcomere length can result large changes in tension generation. 59, 62, 63 Therefore, increasing the sarcomere length enhances the tension development, which affects the changes both in preload condition and the contractility of the heart.

TnT and Alpha and Beta-Myosin Heavy Chain $(\alpha, \beta-\mathrm{MHC})$ are proteins involved in cardiac sarcomere function as discussed previously. TnT is one of three troponins binding with tropomyosin. TnT affects the function of thin filaments and the interaction between the thin and thick filaments, regulating cardiac muscle contractility. ${ }^{50}$ Abnormality of this TnT gene can cause sudden death for cardiac patients. ${ }^{45}$

Myosin heavy chain (MHC) is a major protein expressed in heart tissue during the developmental stage and is responsible for mechanical performance of the heart, especially muscle contractility. ${ }^{64-66}$ In higher vertebrates, two cardiac isoforms of MHC predominate. $\beta$-MHC is expressed mainly during embryogenesis or heart diseases while $\alpha-\mathrm{MHC}$ exists dominantly in the adult heart tissue and inhibits the expression of $\beta-\mathrm{MHC}$. The temporal expression of $\alpha$ and $\beta-\mathrm{MHC}$ is species specific. Interestingly, the distribution of $\alpha-\mathrm{MHC}$ expression is correlated with beating rate. ${ }^{67}$ 
In addition, the deformation of the cytoskeleton can change the physical properties of the cells and distort the nucleus, as well as the biochemical and molecular events inside the cell, such as gene expression, transcription, translation and protein synthesis. This change also affects physical and chemical communication between cells. Therefore, the structure inside the cell, morphology of cell, and the biochemical aspects of the cell are altered as a result of mechanical stimulus.

To respond to any abnormal condition of mechanical stimulations, such as overpressure or overstretch, the heart begins to remodel itself. The remodeling of the hearts causes an increase in the number of fibroblasts, thickens the heart muscle wall, or enlarges the size of the hearts. ${ }^{61,68,69}$ These changes play critical roles in contractility of the cardiac tissue. If the abnormal mechanical stimulus persists, heart remodelling can lead to the cardiac dysfunction (Fig. 1.7)

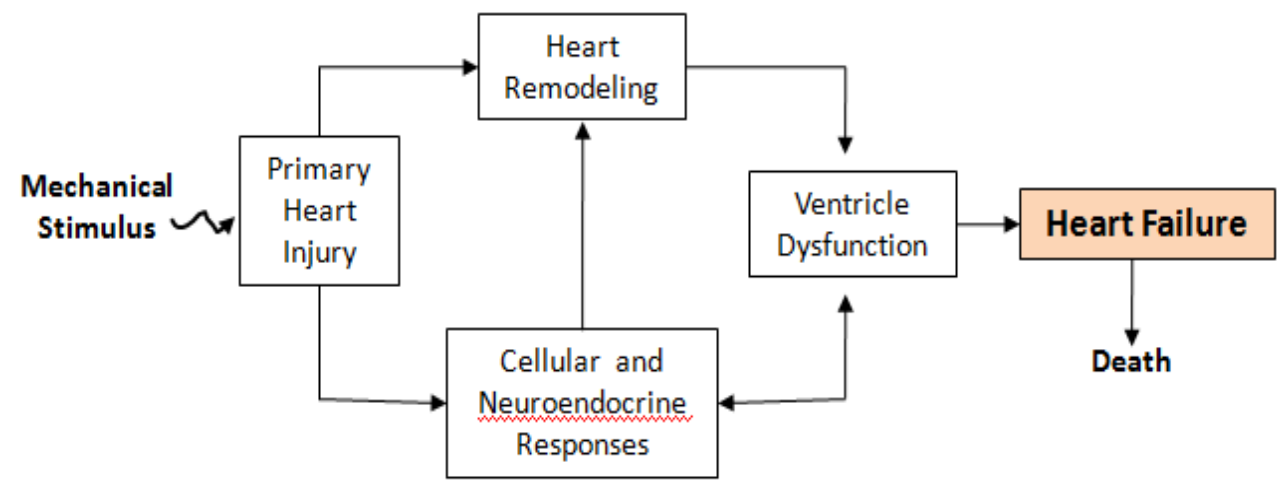

Figure 1.7: Maladaptive responses due to abnormal mechanical loading leads to the heart failure. 


\subsection{CONCLUSION}

The heart is the central organ within the cardiovascular system and is extremely complex in terms of construction and function. This chapter serves to provide an overview of CVD and the heart structure and function. CVD is a serious health problem and continues to claim millions of lives each year while also diminishing quality of life for several others. However, current treatment options, though beneficial have several limitations. In order to improve the quality of life, researchers therefore continue to search for better options to prevent or treat the CVD. 


\section{CHAPTER 2}

\section{IN-VITRO MODEL SYSTEMS TO STUDY CARDIAC TISSUE}

\subsection{NEED FOR CELLULAR-LEVEL STUDIES}

The human body consists of sequential levels of structural and functional organization. Cells are the smallest functional units capable of sustaining themselves and consist of various molecular constituents, including bio-molecules like DNA, RNA and proteins. Cells are organized together to form tissue. Organs in the body responsible for specialized functions are made up of one or more types of tissues. Various organs and/or tissues in the body are organized together to form systems (Ex: Cardiovascular, Nervous, or Circulatory). Finally, an organism sustains life due to synergistic working of various systems within the body. Several mechanisms exist to provide feedback and maintain the body's functions within a tight range (homeostasis).

The tradeoff between generations of highly specific molecular information and translation of that information in-vivo necessitates investigation at various levels of cellular organizations (Fig.2.1). Cell-based studies are essential to provide highly specific information relating to molecular mediator and the targets for drug discovery, therapy, and tissue generation. Analysis of purified or isolated cell populations free from surrounding cellular and extracellular constituents allows the investigation of sub-cellular

mechanisms in great detail, which is not possible in intact tissues. ${ }^{70}$ Study of 
heterogeneous mixtures of cells (like in a tissue) often results in unnecessary noise, which significantly deteriorates the quality of information attainable.

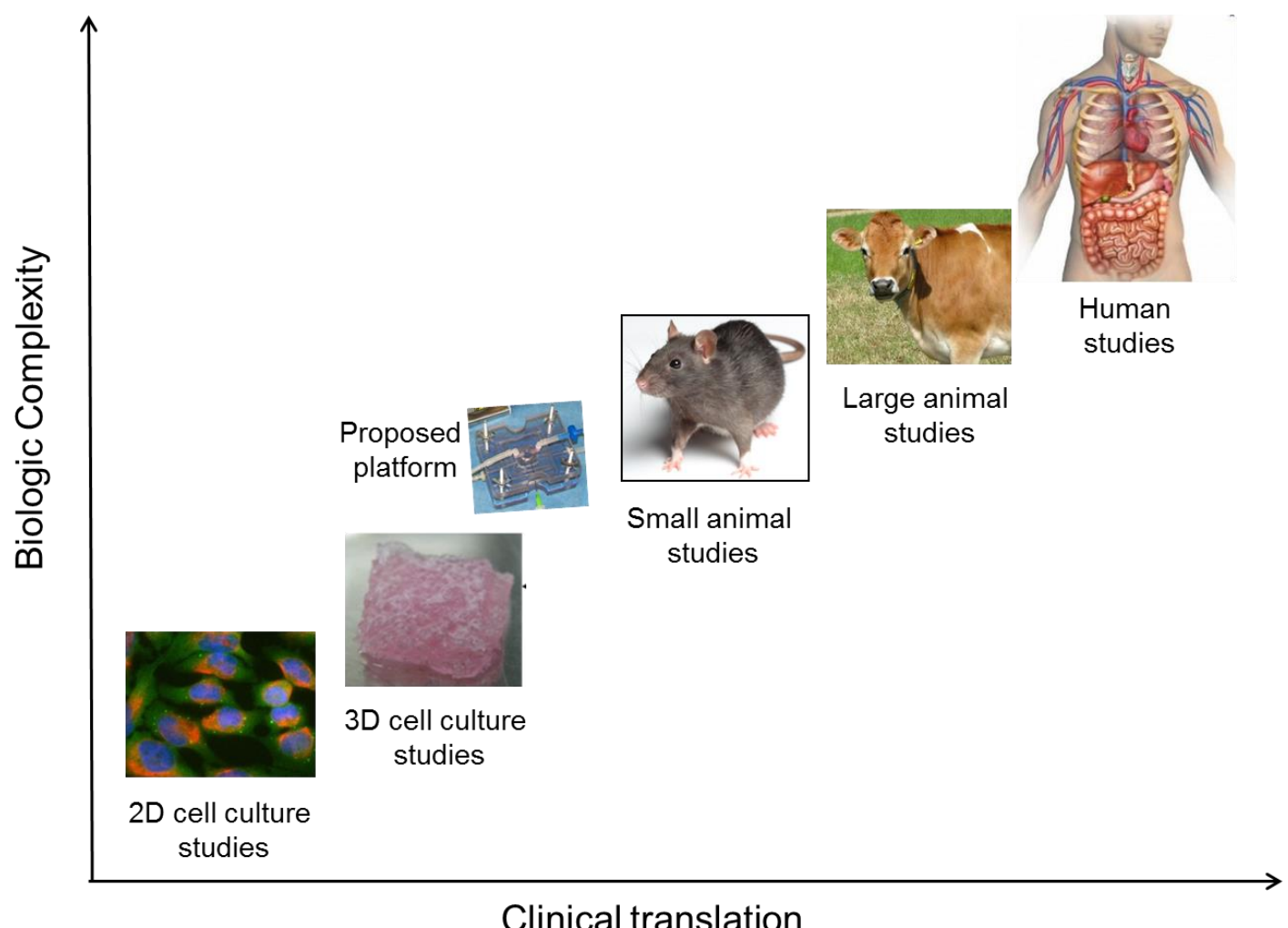

Figure 2.1: Biologic complexity versus clinical translation plot. Human clinical studies are the most relevant, by definition, to clinical translation, but have the least specific whereas cellular level studies provide highly specific information but their relevance to human physiology is low. The in vitro cell cellular model devices seek to significantly enhance the relevance of cellular level studies.

Further, cellular level systems that utilize purified cells, allow for convenient analysis, using both destructive and non-destructive techniques. Cells can be imaged using microscopy to study structure, conformation, the expression of intra and extra-cellular 
markers, organization of organelles and other details of nuclear and cytoskeletal elements. Low and high-throughput techniques can be used to evaluate gene expression, protein expression and production of metabolites. The effect of a drug can be understood in terms of molecular signaling events that alter cell phenotype and function. Highthroughput cell culture, using multi-well plates, can be used to evaluate effects of variables, including concentration of stimulants and time of exposure on cellular function. ${ }^{71}$ The flexibility and specificity of information generated from cellular level systems is unparalleled, and is usually an essential first step in any drug discovery study or tissue generation. However, since cell-based studies use isolated cells in a nonphysiological environment without mechanisms responsible for homeostasis, the relevance of these studies is limited, and results need to be verified using animal and patient level studies.

In-vitro studies are performed in isolation without the biologic complexity and the paracrine feedback mechanisms present in the body. Therefore, to understand the real and long term effects of a biochemical compound, foreign tissue, drug, or device on an organ or in a system, in-vivo evaluation is essential. Animal studies are used to confirm outcomes before application in the human body. Animals are similar in complexity to humans; and the levels of organization are conserved amongst most species. Small animal studies, like study on rats, mice, hamsters and rabbits, are easier to perform due to cost related issues and availability of significant amounts of information and characterization of various systems in animals. ${ }^{61,72}$ Further, technologies for generation of transgenic animals allows for greater flexibility with design of studies to evaluate 
targeted pathways. ${ }^{73}$ Large animals are physiologically closer to humans and provide improved relevance due to greater similarities in size of organs and function of systems ${ }^{74,75}$ Large animals are particularly useful in testing organ/ tissue transplantation or implantable medical devices because the size of their organs is comparable to humans and provides a more accurate representation of the actual situation.

Patient or clinical studies are often the last stage of evaluation for a particular drug or treatment. This follows pre-clinical studies accomplished using in-vitro methods and invivo animal studies. Clinical trials allow assessing the safety and effectiveness of a new medicine or device on patients. Clinical studies are divided into phases $0,1,2,3$, and 4 . Phase 0 trial allows an early evaluation of the pharmacokinetic effects of the candidate drug in humans. This is done by administrating a low dose of the test compound in a short time period. This phase 0 trial doesn't have a therapeutic or treatment purpose; rather, allows the researchers to explore the pharmacological profile of the test compound in human to see if it is appropriate for humans or not. ${ }^{76}$ As a result, it helps to select the potential drug. The disadvantage of this phase is the poor relation between the preclinical and clinical effect of the testing drug. ${ }^{77}$ Phase 1 is the first stage to test the dose effects on human subjects, so the appropriate dose of a certain drug on humans can be found. Phase 2 trials assess how well the drug works and continues to affirm the safety of the drug on large groups. Phase 3 trials are applied on a huge group to see how effective the drug is for a certain type of disease. And finally, phase 4 post-marketing surveillance trials determine potential unanticipated effects of the drug in broader, unselected populations. 
In conclusion, cell-level studies are highly specific and can be used to investigate molecular mediators and signaling pathways involved in disease processes. These studies cannot be accomplished in patients because of inherent difficulties with isolation of cells

and analytical techniques. Animals provide a more appropriate platform for early evaluation as they provide more opportunities for evaluation. To shorten the time between discovery and clinical validation, it is essential that cell-based studies are accomplished in platforms that have physiological relevance. For example: studying cardiac tissue under unloaded static conditions is undesirable since mechanical loading and fluid flow play an important role in maintaining cell structure and phenotype.

\subsection{IMPORTANT CONSIDERATIONS FOR CELL-LEVEL METHODS OF CARDIAC TISSUE DEVELOPLMENT AND CHARACTERIZTION}

During normal heart function, the myocardium experiences passive stretching and increasing transmural pressure during filling (preload) and actively generates mechanical force with further increase in wall stress during ejection (contraction). Cells in cardiac tissue therefore are under constant physical stimulation and rely on the conversion of these cues into intracellular signals to control cell phenotype and muscle mass during physiologic and pathologic conditions. ${ }^{78}$ Cardiac cells therefore are exposed to periodic stress, strain, and shear.

Physiological heart development and cardiac function rely on the response of cardiac tissue to mechanical stress signals during hemodynamic loading and unloading. These 
stresses manifest themselves via changes in cell structure, contractile function and gene expression. ${ }^{72}$ Disruption of this well-balanced stress-sensing machinery due to various pathological conditions results in mechanical dysregulation, cardiac tissue remodeling and heart failure. To achieve physiological relevance, in-vitro models must replicate the complex, dynamic in-vivo interactions between cardiomyocytes and mechanical stress signals. However, current in-vitro models do not incorporate all aspects of mechanical loading and therefore fail to accurately mimic the in-vivo cardiac microenvironment.

\subsection{REVIEW AND CURRENT STATE OF THE ART}

Several animal model studies have proved that hemodynamic mechanical stimulations play significant roles in shaping the formation and ensuring proper heart functions. ${ }^{19,79-84}$ Therefore, for cardiomyocyte differentiation into functional cardiac tissue, in-vitro mechanical stimulation should be included during in-vitro cardiac tissue regeneration.

Various clinical studies for cardiac tissue regeneration via transplantation of progenitor or differentiated cell population have been established. ${ }^{85-87}$ However, the goal of tissue replacement with functional myocardium has not accomplished because a majority of studies apply the direct delivery of undifferentiated cardiac cells to the injured locations. $^{88}$ In addition, the current state of cardiovascular in-vitro models is predominantly cultured under conventional static conditions. ${ }^{69,70,89}$ Most studies utilize isolated cardiomyocyte cells maintained in two-dimensional culture forming randomly oriented cell-extracellular matrix (ECM) interactions. ${ }^{73,90,91}$ Culturing cardiomyocytes in a $2 \mathrm{D}$ system does not reflect the physically biological environment that the in-vivo 
cardiomyocytes experience. As a result, the phenotype and behavior of these 2D cultured cardiomyocytes appear differently from the cardiomyocytes in the in-vivo normal conditions. ${ }^{92}$ Cardiomyocyte grown on the $2 \mathrm{D}$ condition shows flattened, stellate-shape phenotype instead of a rod-shape phenotype as it is in the in-vivo environment. Proliferation rate, differentiation, and DNA synthesis are poor. ${ }^{16,93,94}$ The interaction between cell-cell or cell-matrix are limited due to the 2D planar geometry; thus, cell-cell connections are loose, which fails to initiate spontaneous contraction.

There are groups using 3D engineered cardiac constructs to investigate cardiomyocyte maturation, response to drugs, and the effects of genetic defects in ion channels and sarcomeric proteins. The phenotype and molecular regulation and the function of cardiomyocytes in 3D shows more likely resemble the in-vivo cardiomycytes. ${ }^{16,92-100}$ However, there are a limited number of bioreactors that can generate 3D tissue on a scale relevant for clinical translation. Complex systems that can replicate in-vitro mechanical stimulation occurring in the heart are currently unavailable. Accurate replication of the in-vivo environment requires consideration of several factors. First, tissue orientation and direction of stretch are critical determinants of cardiac cell signaling; therefore randomly oriented cultures cannot replicate physiological loading. Second, fluid flow is an important factor as shear stress strongly influences endothelial and smooth muscle phenotype but not cardiomyocytes. Third, cyclic build-up of pressure and stretch during preloading are critical determinants that dictate cell structure and function; hence, perfused systems are more representative of the in-vivo environment. Fourth, 
synchronous contractions ensure ejection of fluid that in turn results in relaxation of builtup pressure and stretch. ${ }^{38}$

Some of the aforementioned issues have been previously addressed, albeit individually. Patterning techniques like micro-contact printing and lithography to define areas of cell attachment and directional alignment have been accomplished. ${ }^{101-103}$ Several groups have cultured cardiac cells under continuous and cyclic loading conditions to mimic in-vivo hemodynamic loading. ${ }^{104-107}$ Commercial technologies like Flexercell ${ }^{\circledR}$ Strain -FX-4000 Flexercell Tension Plus ${ }^{(\mathrm{TM})}$ (http://www. flexcellint.com), which consists of a flexible membrane on which cardiac cells can be plated and then be subject to oscillatory deflections using a vacuum or pressure source, have been used to apply mechanical stretch. $^{96,108}$ Electrophysiological studies utilize systems such as the IonOptix Myocyte Systems (http://www.ionoptix.com) where cardiomyocytes grown on a glass slide are stimulated with an electrical impulse and analyzed for contractility. However, to the best of our knowledge, no in-vitro system currently replicates all aspects of cardiac mechanical loading and unloading in the heart.

\subsection{PHYSIOLOGICALLY RELEVANT SYSTEMS FOR CARDIAC CELL}

\section{CULTURE}

Various manifestations of cardiovascular diseases directly correspond to changes in mechanical behavior of cardiac cells. This, in turn, affects cellular phenotype and function, causing changes like hypertrophy, growth of fibrous tissue, and establishment

of random cellular orientation. ${ }^{69}$ For example, in hypertension, the pressure inside the 
heart exceeds the normal range due to a high resistance in vascular system and eventually results in hemodynamic overloading of the heart and vasculature. This higher intracardiac and transmural pressure triggers myocytes hypertrophy initially and subsequently can lead to a dilated phenotype associated with myocytes loss. In the long term, this additional stretch weakens the heart muscle, causing heart failure. ${ }^{61}$ In the contrast, hypotension refers to the condition where the pressure inside the heart is lower than the normal range due to abnormal filling or ejection or where the arterial vasculature is abnormally dilated. This causes reduced blood circulation and oxygen deprivation to the rest of the body. In response to this oxygen deprivation and reduced nutrient delivery, the heart rate increases, resulting in altered stretch and pressure profiles.

The frequency of the loading determines the ranges for normal and abnormal heart function. For example, in human, bradycardia is a condition where the heart rate is lower than $60 \mathrm{bpm}$, and tachycardia is another condition characterized by a significantly higher heart rate (>200 bpm) in comparison to normal heart rate $(\sim 75 \mathrm{bpm})$. In response to these abnormal patterns, the heart either lowers or increases the systolic fraction. ${ }^{109}$ All of these changes can cause the reorganization of cardiac tissue and it can lead to heart failure or sudden death if it persists long term. ${ }^{110}$

Finally, due to fluid flow, shear stress can be a significant mechanical cue for cardiac endothelial and cardiac smooth muscle cells. Cardiac endothelial cells contribute to the internal lining of the myocardium and are directly exposed to fluid induced shear stress during hemodynamic loading and unloading. Changes in magnitude, frequency, or 
duration of shear stress are crucial for the structure, function and permeability of endothelial cells. Various clinical conditions occur as a result of changes to shear profiles seen by cardiac endothelial cells.

Studying cardiovascular disease requires appropriate cellular level platforms which can be used to culture cells while accurately mimicking mechanical and fluidic loading conditions seen in-vivo. In cardiovascular disease, various conditions are associated with distinct mechanical loading profiles and replication of those profiles is critical to ensure proper understanding at the cellular and molecular levels. Mechanical loads experienced by cardiac cells vary as a consequence of dysfunction including pressure, stretch and shear stress, as well as the frequency and duration.

\subsection{IN-VITRO TECHNIQUES FOR MECHANICAL STIMULATION OF CELLS}

Cells in different tissues experience cyclic compressive loads. Cardiac and vascular tissues are examples of tissue where cells within the tissue are constantly under compressive loading. Replicating this condition in-vitro can be accomplished using different techniques such as application gas pressure (hydrostatic compression) directly to the culture system or through the fluid induced loading of the system (pressure loading). These techniques cause direct application of pressure to cells in culture mimicking conditions seen in-vivo.

Another commonly seen loading condition in cardiac and vascular tissue is cyclic stretch (and strain). The most common approach to stretch the cells in culture is by growing the 
cell on a flexible surface and applying stretch on the surface where the cells are attached. The stretch can be uniaxial, biaxial, and include shear. With the uniaxial stretch, the surface is either rectangular or square and is stretched in one direction either longitudinally to both ends, or is bent/flexed in a four-point bending. With the biaxial stretch, the membrane is circular and the edge of the membrane is fixed. The applied pressure deforms the membrane in both the radial and circumferential directions. However, the strain depends heavily on the location on the membrane, causing the cells on different locations to stretch differently. For example, cells seeded at the center will stretch more than those at the edges. As a result, it is extremely complicated to analyze the data and to interpret the cells behavior. To produce the equi-biaxial strain, the membrane has to slide non-frictionally on a stable plane. On this plane, the stress distribution on the area of the membrane where it is supported by the plane, is symmetrical, creating an isotropic and spatially constant strain.

Finally, cells in different tissues experience shear stress as a consequence of fluid flow. Shear stress is a key mediator of cells phenotype. Shear stress controls alignment and establishes cell-cell contacts, which are important in the formation of tight junctions in ensuring low permeability in vasculature. The velocity, as well as the pattern of the flow, laminar versus turbulent flow for example, can create different shear stress on the cells. Also, viscosity of the fluid is another factor that can increase or decrease shear stress on the cells. 
In cardiac and vascular tissue, all three of these loads (cyclic pressure, stretch, and shear stress) are experienced simultaneously. Additionally, changes in one or more of these loads have a cascading effect on the entire system. Therefore in-vitro models of cardiac tissue need to mimic accurately the combined effects of cyclic pressure, stretch and shear in a pulsatile fashion.

\subsection{CONCLUSION}

Based on the discussion above, due to the complex nature of the heart and the importance of mechanical loads, an in-vitro cellular culture model designed with physiological relevance is critical to investigate the cellular and molecular adaption of cardiac cells and tissues relevant to cardiovascular adaption and disease. No device or cell culture platform is commercially available today to accomplish long term culture of cardiac cells in-vitro with accurate replication of realistic mechanical loading profiles. Efforts by other groups thus far have succeeded in replicating isolated mechanical loads. There is still a need for platforms where the culture conditions can be dynamically varied not only to replicate normal loading conditions, but also abnormal loading conditions. These platforms are necessary to study the molecular pathophysiology of cardiovascular diseases and in the discovery of drugs, treatment options and cardiac cell regeneration strategies. 


\section{CHAPTER 3}

\section{THE $\mu$ CCCM SYSTEM}

\subsection{INTRODUCTION}

Understanding the molecular basis of manifestations of cardiovascular disease requires multiscale and multilevel approaches and an appreciation of the developmental mechanisms that regulate cardiac morphogenesis and remodeling. Because cardiomyocyte cell death is the major cause of cardiac pathology leading to heart failure, many efforts are underway to restore cardiac tissue structure and function through regenerative approaches. ${ }^{69}$ Unfortunately, unlike most cells in the body, cardiomyocytes stop differentiation and have limited proliferative potential after birth in higher vertebrates. ${ }^{101}$ To accommodate, scar tissue replaces the damaged cardiac tissue. Scar tissue increases the stiffness of the heart tissue and cause the inability to regenerate the same contractile forces as the normal undamaged cardiac tissue does. Therefore, cardiac tissue engineering plays a significant role in the process to find better treatment of heart disease due to the loss of cardiomyocytes. However, replicating all aspects of the mechanical loading and unloading environment for the in-vitro cardiac cultured cells is a challenging task as discussed in chapter 2. It is because the myocardium experiences passive stretching and pressure build-up during filling (preload) and actively generates

mechanical force during ejection (contraction) against variable afterload. Cells in cardiac tissue are under constant physical stimulation and rely on conversion of these cues into 
intracellular signals to control cell phenotype and muscle mass during mechanical conditions. Therefore, to study the molecular pathophysiology of cardiovascular diseases, in the discovery of drugs, treatment options, and cardiac cell regeneration strategies, invitro cardiac tissue must be cultured in the in-vivo like environment.

The need for platforms where the culture conditions can mimic the physiologically mechanical condition and can be dynamically varied to normal and abnormal environments necessitates engineers to design different cell culture models. With a primary goal of replicating such in vivo mechanical loading conditions for in-vitro cardiomyocytes and to enable cardiac tissue generation, a microfluidic cardiac cell culture model $(\mu \mathrm{CCCM})$ was developed. ${ }^{111}$ The first generation of the cardiac cell culture model was built in 2008 in order to mimic cyclic mechanical strains and pressures experienced by cells during the cardiac cycle. With this model, cardiac cells undergo cyclic stretch and contraction, pulsatile pressure, as well as fluid loaded and ejected.

\subsection{THE $\mu \mathrm{CCCM}$}

The $\mu \mathrm{CCCM}$ system consists of a peristaltic pump, cell culture chamber, pulsatile actuated collapsible valve, and a tunable resistance in a series, along with accessories including tubing, an oil chamber, a pressure generator, and a digital manometer. (Fig. 


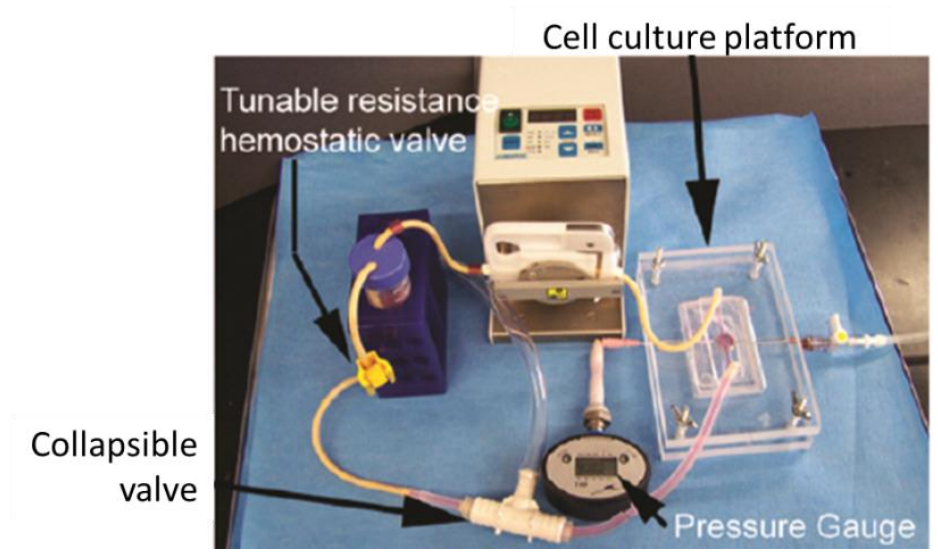

Figure 3.1: Assembled $\mu \mathrm{CCCM}$ for cell culture experiment. The tunable valve and collapsible valve were placed downstream of the cell culture platform to create the resistance and pulsatile pressure inside the cell chamber. Cell culture chamber (pink color) was clamped between two fixtures of the cell culture platform. The $\mu \mathrm{CCCM}$ system has a closed circulating fluid network.

Cells were placed in culture on a thin PDMS membrane attached at the bottom of the cell culture chamber inside the platform. Continuous circulation of culture medium is maintained using a peristaltic pump. Downstream the chamber is a collapsible valve actuated in a pulsatile fashion using a pressure generator. Closure of this collapsible valve leads to pressure build up in the chamber, which in turn also leads to stretching of the thin membrane as well as the cells on this membrane. This stretching phase mimics the diastolic loading phase in the ventricle. To ensure uniformity, a post is placed beneath the thin membrane such that, during stretch, the portion of the membrane on which cells are cultured experiences uniform strain and the edges experience larger nonuniform strain (Fig. 3.2). Lubricating oil fills in the chamber beneath the cell culture area to create friction-free sliding of the membrane on the post. Further, to influence outflow 
resistance, a tunable homeostasis valve is placed downstream of the collapsible valve to mimic afterload. Fluid transport and subsequent shear stress can be controlled by varying the pump flow rate. Pressure buildup and strain can be controlled via a combination of factors, including the thickness of the membrane, fluid flow rate, and operation of both valves
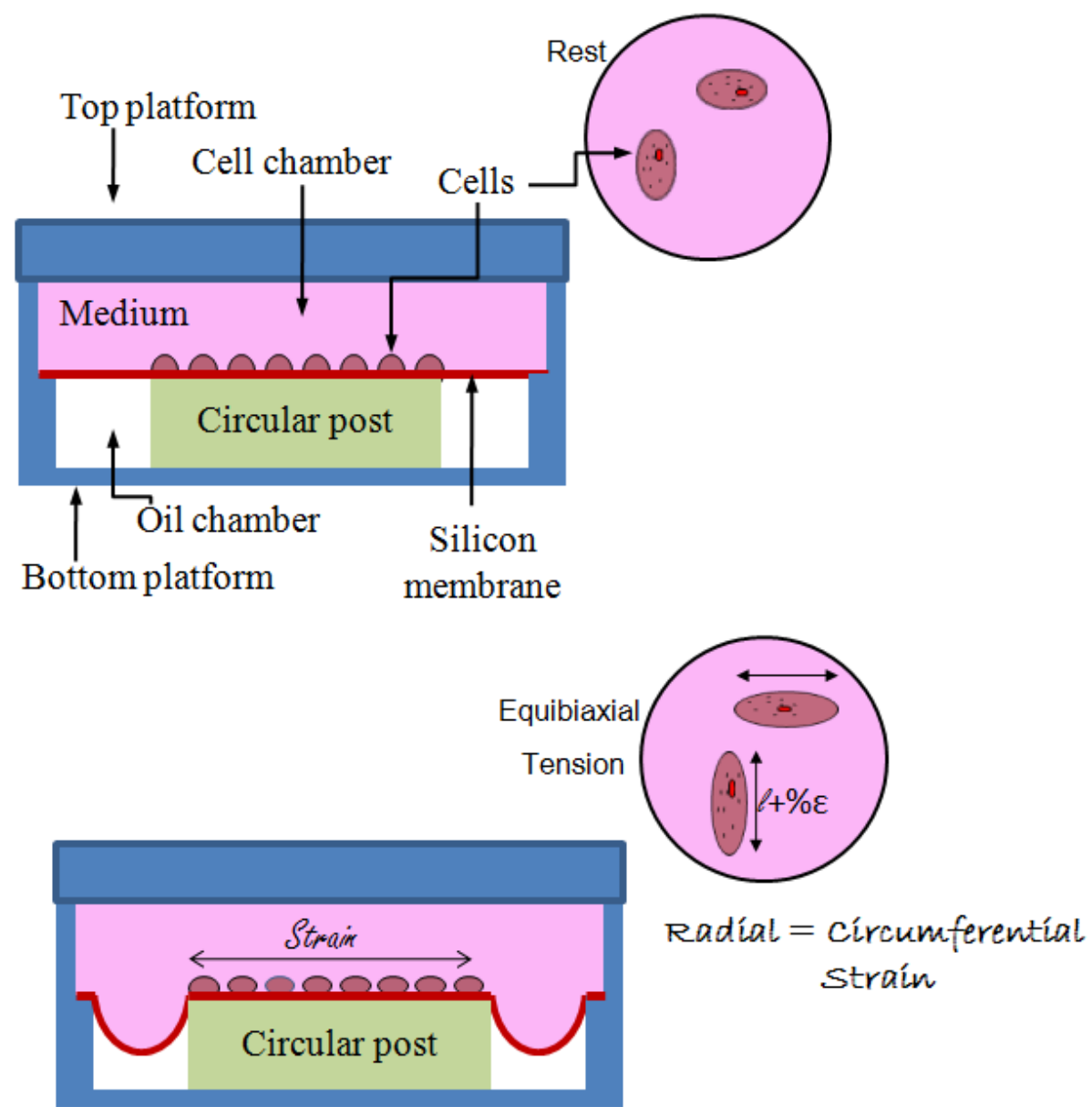

Fluid loading induced pressure

Figure 3.2: Setup design for equi-baxial strain. A significant deformation at the edges of the membrane occurs when the pressure is applied, but the area of the membrane rested on the post has the same radial and circumferential strain. 


\subsection{FABRICATION AND ASSEMBLY OF THE $\mu$ CCCM}

\subsubsection{Assembly for Housing the Cell Culture Platform and the $\mu \mathrm{CCCM}$ components}

The assembly for housing the cell culture platform was fabricated in the Rapid Prototyping Facility at the University of Louisville using Computer Numerical Control (CNC) Milling Machine. The designs were laid out using Solidworks 3D layout software prior to machining the assembly using $\mathrm{CNC}$ milling. Acrylic was used for construction. (Fig. 3.3)

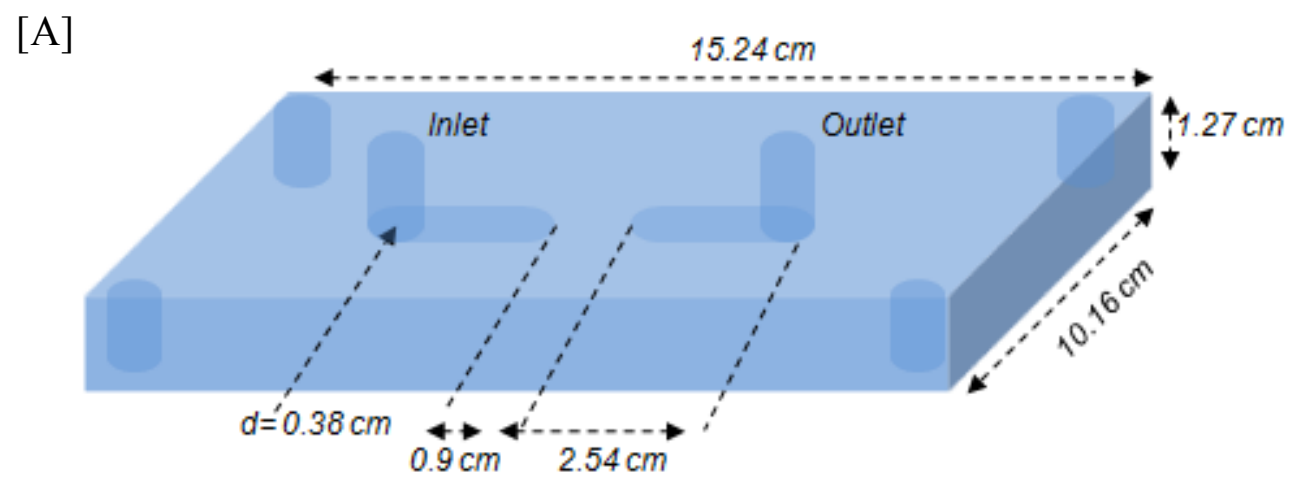

[B]

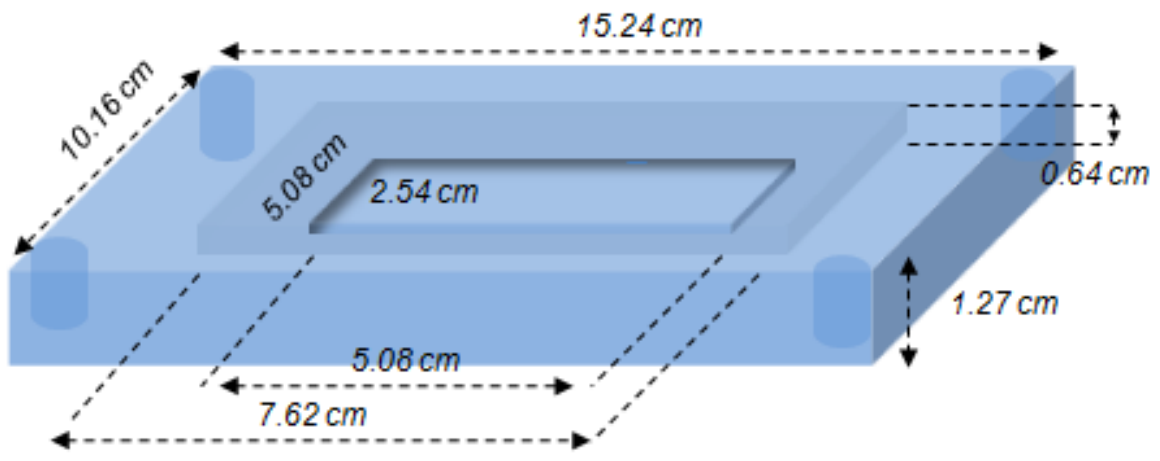

Figure 3.3: Schematic representation of the top and bottom fixtures of the platform for housing the cell culture chamber. [A] The top fixture has the fluid inlet and outlet accesses; [B] The bottom part of the platform has two rectangular holes at the center. 
The cell culture chamber ( $\sim 1 \mathrm{~cm}$ diameter) and oil chamber were made from polydimethyl siloxane (PDMS) (Dow Corning, ratio 1:10 of the curing agent and the base) using standard soft-lithography techniques. The collapsible valve was created mainly via thin elastic ARGYLE Penrose tubing. More detail information for these fabrications will be seen in the published article. ${ }^{111}$

\subsubsection{Assembly of the $\mu \mathrm{CCCM}$}

First, an acrylic sheet was placed in the rectangular hole on the bottom piece of the platform, followed by the oil chamber (Fig.3.4). Then, the cell culture chamber, with $18 \mathrm{G}$ needle inserted, was aligned on top of the oil chamber. The top piece of the platform was then laid on top of the cell chamber. The platforms were tightened with four screws. Finally, low viscosity oil was injected into the oil chamber through needles.

A 3-stop Pharmed BPT tube (1.3 mm ID, Cole-Parmer, Vernon Hills, IL) was connected from a $50 \mathrm{~mL}$ medium container to the inlet of the device via a peristaltic pump (Ismatex, ISM 596D, Vancouver, MA). The collapsible valve was connected from the outlet of the device to the medium container. The tunable valve was placed downstream of the collapsible valve (Fig.3.1.). Finally, the pressure sensor was connected to the needle,

which was connected to the cell chamber. A pulsatile pressure generator was connected to the bottom $T$ of the collapsible valve. 


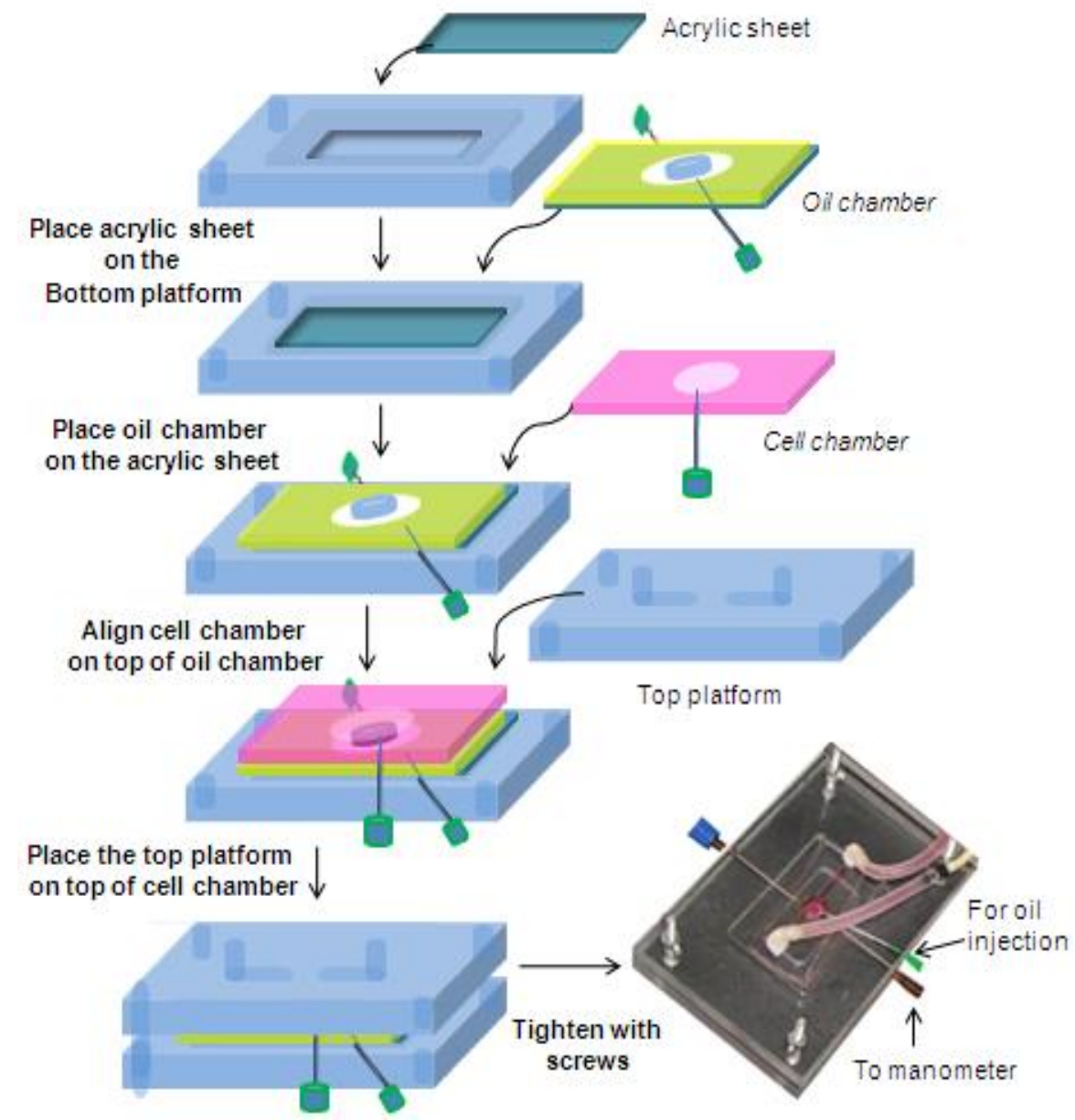

Figure 3.4: Assembly of the cell culture and oil chambers between the platforms.

\subsection{OPERATION OF THE $\mu$ CCCM}

Once assembled as in Fig. 3.1, the peristaltic pump was started gradually from a low flow rate to the desired flow rate. After 30 min running, the pressure in the pneumatic pressure generator source was applied and the tunable valve was adjusted until the pressure reached the desirable values. Variable parameters, such as the frequency of the membrane stretching as well as the percentage of systolic phase per one cycle, were determined based on the requirement of each experiment. The frequency of the stretching was controlled through the $\%$ systolic and the frequency controls on the 
pressure generator source. The pressure inside the cell chamber was varied by the corroboration between the pressure applied on the collapsible valve and the adjustment of the tunable valve. The stretching level of the membrane was determined by the thickness of the membrane, which were measured and standardized appropriately and the pressure applied on the membrane. And the shear stress on the cells was controlled by the velocity of the fluid loading and the dimension of the cell culture chamber.

\subsection{APPLICATIONS}

\subsubsection{Pressure profiles}

The device was assembled and the pressure build-up within the chamber was generated for the following clinically relevant experimental conditions - normal, heart failure, hypertension, hypotension, tachycardia, and bradycardia. To accomplish pressure monitoring within the chamber, a high-fidelity pressure catheter (Millar Instruments, Boston, MA) was inserted through a hemostatic valve and syringe needle such that the pressure sensing element resided in the center of the chamber. The chamber pressure waveforms were analyzed by using a Hemodynamic Evaluation and Assessment Research Tool program developed in Matlab (MathWorks, Natick). The different waveforms for various physical heart conditions were therefore replicated and recorded by either increasing or decreasing the pressure in the pressure generator and by adjusting the tunable valve (Fig. 3.5). These results confirmed that the $\mu \mathrm{CCCM}$ enabled to regenerate various clinically relevant physiologic conditions of the heart. 

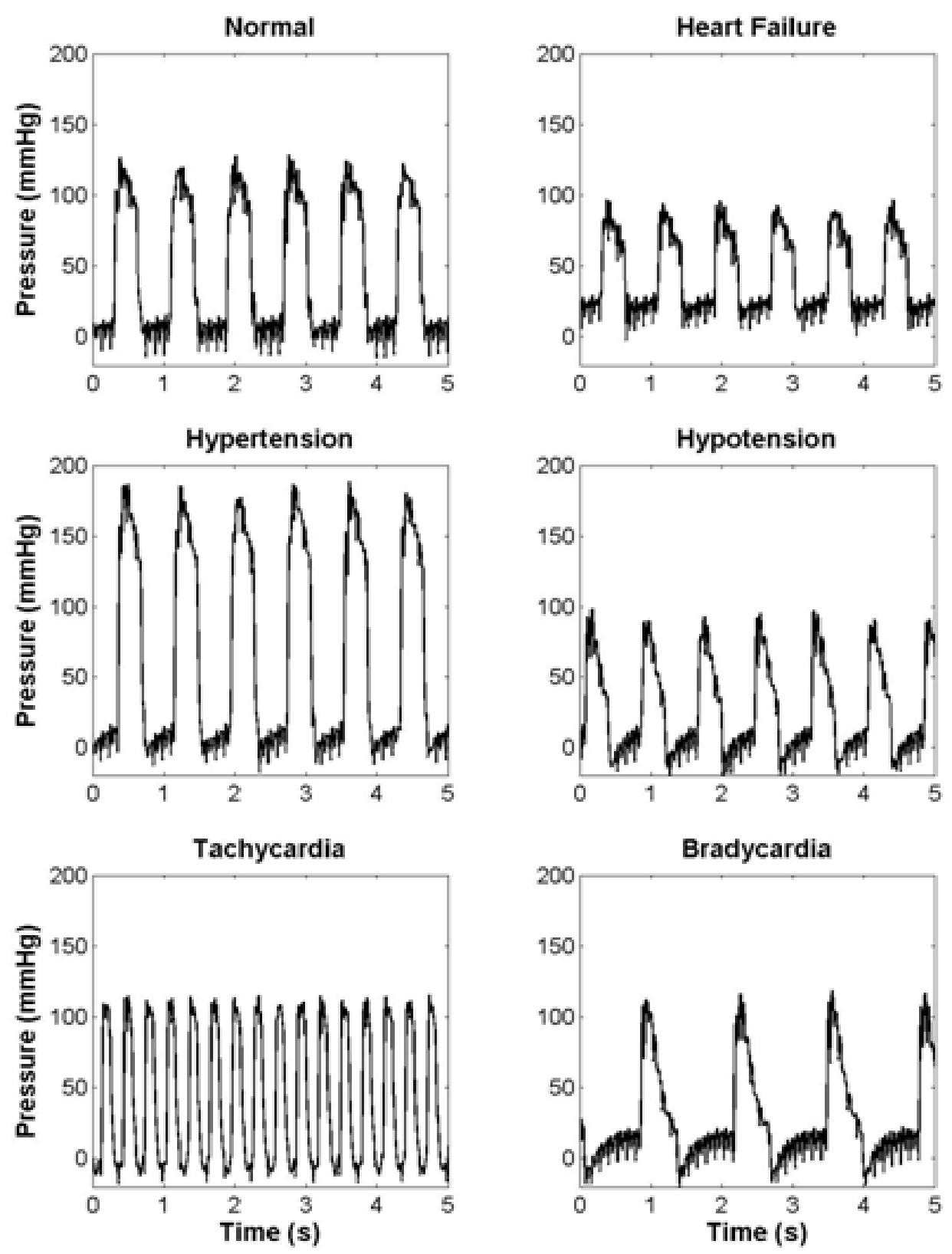

Figure 3.5: Pressure waveforms that simulate various physiologic conditions in the $\mu \mathrm{CCCM}$. These conditions include both normal and dysfunctional myocardium, such as heart failure, hypertension, hypotension, bradycardia, and tachycardia. 


\subsubsection{Cell studies for the proof-of concept}

$\mathrm{H}_{9} \mathrm{C}_{2}$ cells were cultured within the $\mu \mathrm{CCCM}$ system to demonstrate the proof-of-concept. $\mathrm{H}_{9} \mathrm{C}_{2}$ cells were cultured in static condition (control) and within the $\mu \mathrm{CCCM}$ under pulsatile pressure and stretch, representing normal physiological loading (peak pressures of $120 \mathrm{~mm}$ of $\mathrm{Hg}$, strain of $20 \%$ and a frequency of $80 \mathrm{bpm}$ with $50 \%$ systolic to diastolic, flow rate of $8.4 \mathrm{ml} / \mathrm{min}$ ) for $24 \mathrm{~h}$ at $37^{\circ} \mathrm{C}$ and $5 \% \mathrm{CO}_{2}$. More detail of cell culturing is available on the published article. ${ }^{111}$

The results showed that cells not only survived physiological loads but also showed the positive effects of physiological loading. Cells cultured in static condition (control) attained a fibroblast like morphology (Fig. 3.6a), whereas cells cultured in the $\mu \mathrm{CCCM}$ were more rectangular, and presented a postage stamp-like morphology (Fig. 3.6b). The size of the cells cultured within the $\mu \mathrm{CCCM}$ exposed to pressure was larger than those cultures in static condition.

Staining with Phaloidin showed a low intensity fluorescent color and random orientation of f-actin in controls in comparison to aligned f-actin in cells cultured with pulsatile pressure and stretch (Fig.3.6c and d). This indicated that the contraction-stretching of the cells promotes the increasing $\%$ of f-actin and also an increase in the length of the sarcomere, and helps to align the cells in one direction. The increase in the length of the sarcomere is the result of the increase in contraction of the cell under pulsatile stretch. The alignment of the cells demonstrated that mechanical loading spontaneously rearranged the orientation of the cells. 
P-phospholamban was detected in cells cultured within the $\mu \mathrm{CCCM}$, however, the expression of this protein was very low in cells cultured in static condition, as shown is Figure 3.6e, in comparison to the cells cultured in $\mu \mathrm{CCCM}$ (Fig. 3.6). Phospholamban is the integral membrane protein. To respond to the contraction of the cells, phospholamban is phosphorylated. With cAMP, this p-phospholamban promotes the influx of $\mathrm{Ca}^{2+}$ into the cells to bind to the contractile unit, causing the cells to contract. ${ }^{12,}{ }^{113}$ Therefore, indirectly, the increasing of activation of p-phospholamban, which was reflected by the intensive green color on the loading sample, in comparison of the color on the control sample on the Fig. 3.5.e and f, will be indicative of cell contraction. 

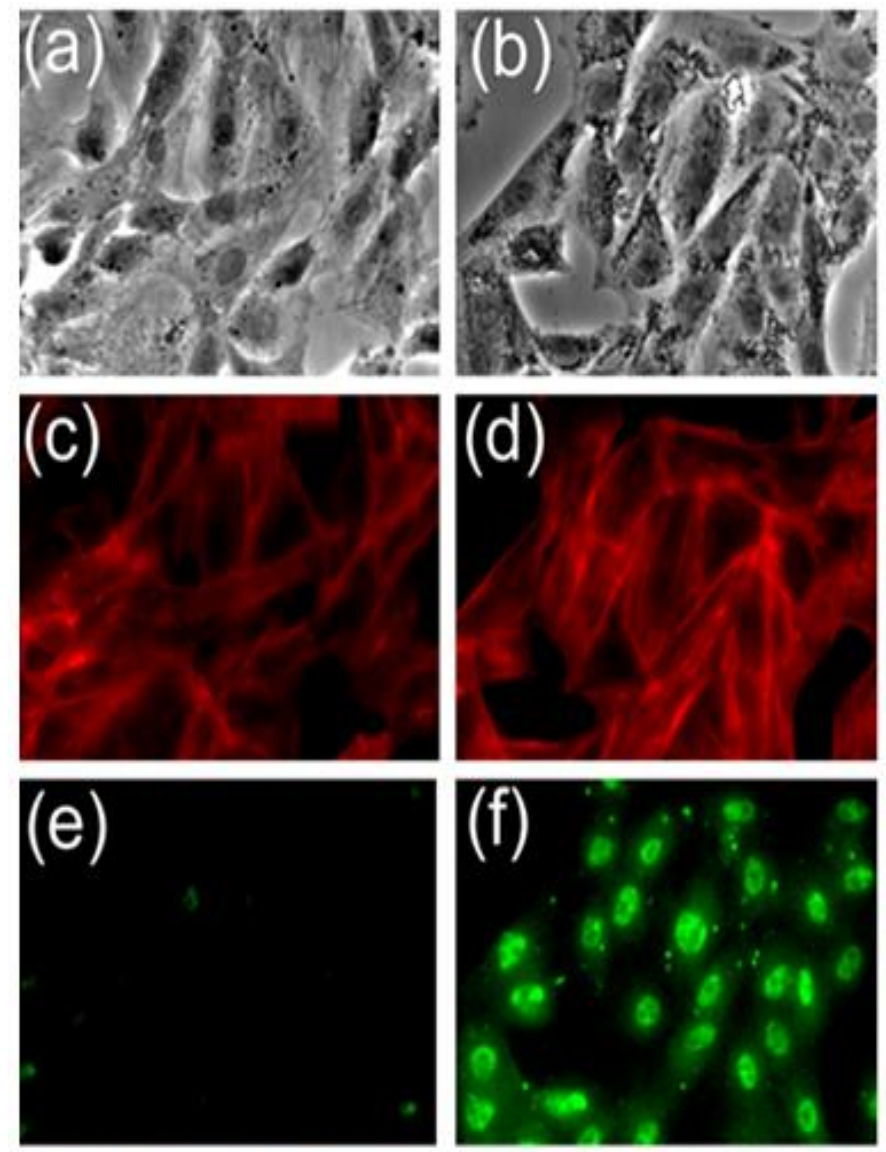

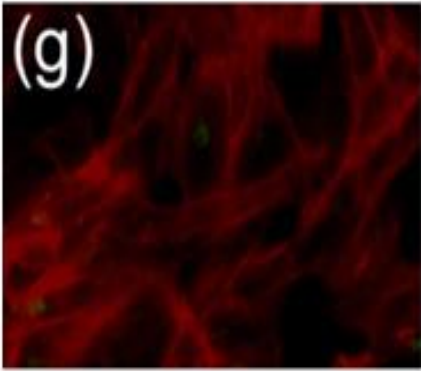

Control

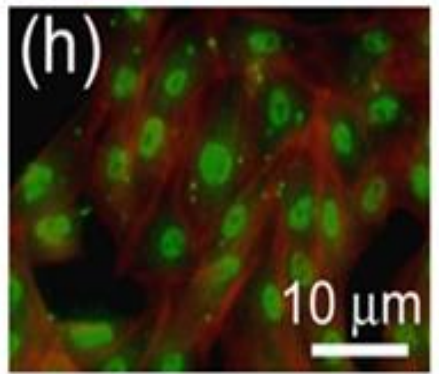

Loading

Figure 3.6: Physiological loading promoted the alignment of f-actin and increased phosphorylated phospholamban activity in $\mathrm{H}_{9} \mathrm{C}_{2}$ cells. Cells were under a static condition (control) and normal physiological loading for 24 hrs. Notice the directional alignment of f-actin stress fibers and increased p-phospholamban activity in the loading condition. (a, b) Phase contrast images; (c, d) Staining with TRITC-conjugated Phaloidin 
for detection of f-actin; (e, f) Immunofluorescence staining for detection of $\mathrm{p}$ phospholamban activity; (g, h) Merged images of F-actin and p-phospholamban.

The proof-of-concept demonstrations using the $\mu \mathrm{CCCM}$ system for cardiac culture were accomplished, confirming that this system has the ability for the design and study of various conditions with different cell types associated with CVD. These studies can be cardiomyocyte regeneration using mechanical loading and stem cells, adult cardiomyocyte regeneration using controlled mechanical loads, study of cardiac and aortic endothelial cells under conditions of continuous and pulsatile flow ${ }^{114,115}$ and finally, evaluation of drugs and other treatment options using cardiac cells maintained under various disease conditions.

\subsection{CONCLUSION AND LIMITATIONS}

Microfluidics based systems have been used to create cardiomyocyte actuators and pumps as well as cell models. ${ }^{102,} 116-121$ The $\mu \mathrm{CCCM}$ was the first system, to our knowledge, that was developed to replicate the mechanical loading conditions observed in the heart, which includes the fluid flow, pulsatile stretch, and pressure. The $\mu \mathrm{CCCM}$ allowed dynamic studies accomplished via the direct manipulation of flow rate, valve operation, membrane thickness and the height of the cell culture chamber. We envisioned that this system would provide time varying mechanical cues for differentiation of stem/progenitor cells into adult cardiomyocytes and would also provide mechanical conditioning to generate sufficient contractile forces and integrate within native cardiac tissue to augment cardiac function. However, after re-examining carefully 
the working principles of this system, we recognized that the pressure-volume relationships seen this in vitro system did not accurate replicate in vivo left ventricle PV relations. First, in-vivo, the stretch of nature cardiac tissue is mainly due to the fluid loading during the loading phase, while the stretch of the PDMS membrane in the $\mu \mathrm{CCCM}$ is based on the pressure generated inside the cell chamber during the loading phase. Second, the stretch and contract of cardiomyocytes are in 3D directions, which include circumferential and radical stretch. With the post, this $\mu \mathrm{CCCM}$ system creates the $2 \mathrm{D}$ biaxial stretching. In addition, in this model, the cells were seeded on a rigid $2 \mathrm{D}$ surface membrane which potentially constrained cell shortening and does not reflect the physiological condition of the in-vivo tissue. The summaries of similarities and differences between the left ventricle and the $\mu \mathrm{CCCM}$ system are listed on the table 3.1. To overcome these shortcomings, the second generation of the cardiac cell culture model (CCCM) was designed and developed. In this new system, cells were cultured on a soft matrix attached to the top of a thin PDMS membrane in a cell culture chamber. With each cycle, cells cultured within the CCCM underwent passive stretch, isovolumic contraction and pressure buildup, ejection and relaxation. Thus, the second generation of cardiac cell culture model system is essentially a left ventricle mimic that can be dynamically tuned to go from static unloaded state (as seen in very early development), to pulsatile pressure and cyclic stretch conditions seen in the adult heart. 
Table 3.1: Summary of similarities and differences (in italic) between the left ventricle and the $\mu \mathrm{CCCM}$ system

\begin{tabular}{|c|c|c|}
\hline Aspects & Left Ventricle & The $\mu$ CCCM system \\
\hline $\begin{array}{l}\text { Working } \\
\text { principles }\end{array}$ & $\begin{array}{l}\text { - "Filling" phase: fluid in, no } \\
\text { fluid out, no pressure buildup. } \\
\text { - } \quad \begin{array}{l}\text { Isovolumic contraction phase: } \\
\text { pressure inside is compressed, } \\
\text { no fluid in nor out. }\end{array} \\
\text { - Ejecting phase: cavity pressure } \\
\text { exceeds aortic pressure, } \\
\text { pressure peaks and then begins } \\
\text { to decline. } \\
\text { - Isovolumic relaxation phase: } \\
\text { muscle relaxes and pressure } \\
\text { decreases prior to the next } \\
\text { filling cycle. }\end{array}$ & 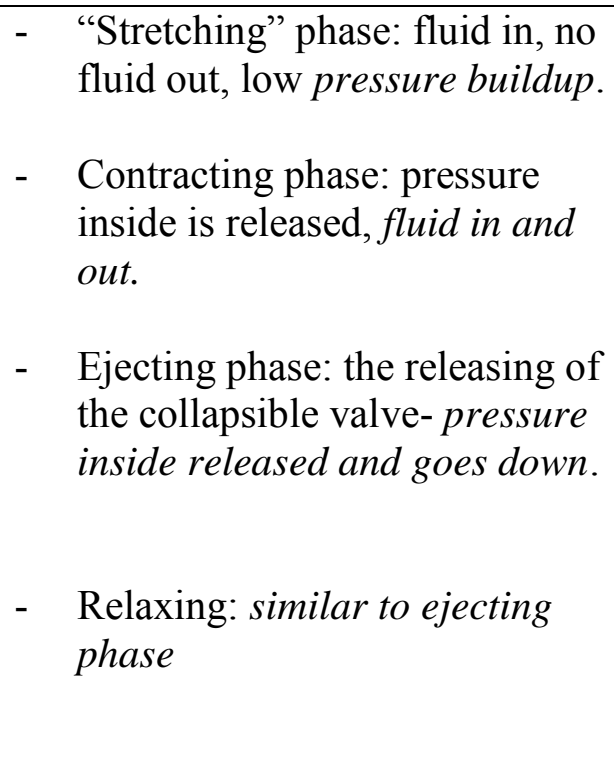 \\
\hline $\begin{array}{l}\text { Circulation } \\
\text { network }\end{array}$ & $\begin{array}{l}\text { - One way valves at the entrance } \\
\text { and outlet of the ventricle }\end{array}$ & $\begin{array}{l}\text { - Only one collapsible valve } \\
\text { downstream of the chamber, } \\
\text { and the fluid continuously } \\
\text { loaded to the cell chamber. } \\
\text { - }\end{array}$ \\
\hline Stretching & - 3 dimensional strains & - $\quad$ Biaxial stretch \\
\hline $\begin{array}{l}\text { Cell } \\
\text { cultured }\end{array}$ & - $\quad$ Soft tissue & - Hard surface \\
\hline
\end{tabular}




\section{CHAPTER 4 \\ THE SECOND GENERATION CCCM SYSTEM}

\subsection{COMPONENTS OF THE SECOND GENERATION CCCM SYSTEM}

The Second Generation CCCM system consists of a fluidic flow pathway, an air pathway, a cell culture platform station, and a control system (Fig. 4.1). ${ }^{122}$

The fluid flow network includes a medium reservoir, a peristaltic pump, a fluid compliance, an upstream one-way valve, a cell culture platform station, a downstream one-way valve, and a tunable pressure relief valve in a series (Fig.4.1). From the medium reservoir, via the assistance of the pump, the fluid flows through the upstream one-way valve into the cell culture chamber and exits the cell culture chamber. The fluid then passes through the downstream one-way valve and the tunable valve and then returns back to the reservoir before being recycled within this flow loop via the peristaltic pump. A bypass with a spring valve was installed at the downstream pathway to prevent the chamber pressure from exceeding the pressure range set up for each case of studies.

While the fluid network is a closed pathway, the air network is a partially closed pathway. The air network includes the cell culture platform station, an air-compliance, a pneumatic valve, and a pneumatic pressure driver (Fig. 4.1). Air from the driver enters 
the cell culture platform station and passes through the air compliance and the pneumatic valve before returning to the pneumatic pressure driver. This air network is a partial closed network because the pneumatic valve is a three-way valve. One of its three ports releases air to the outside when needed.

[A]

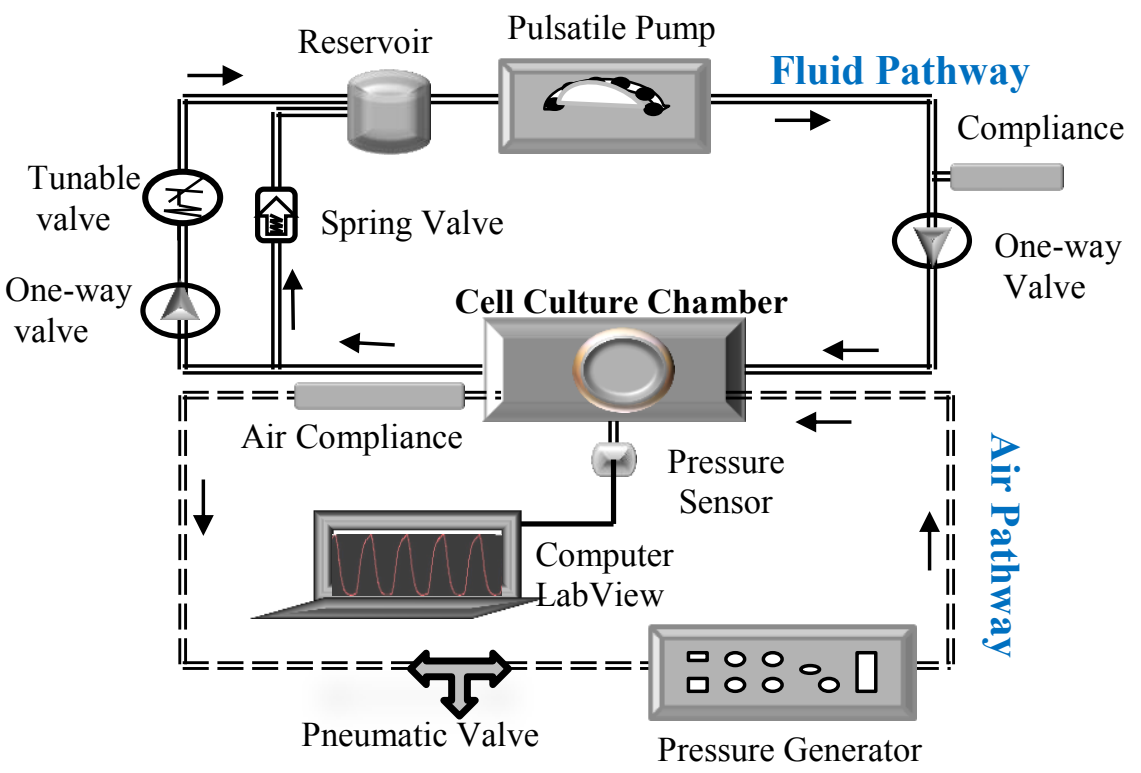

[B]

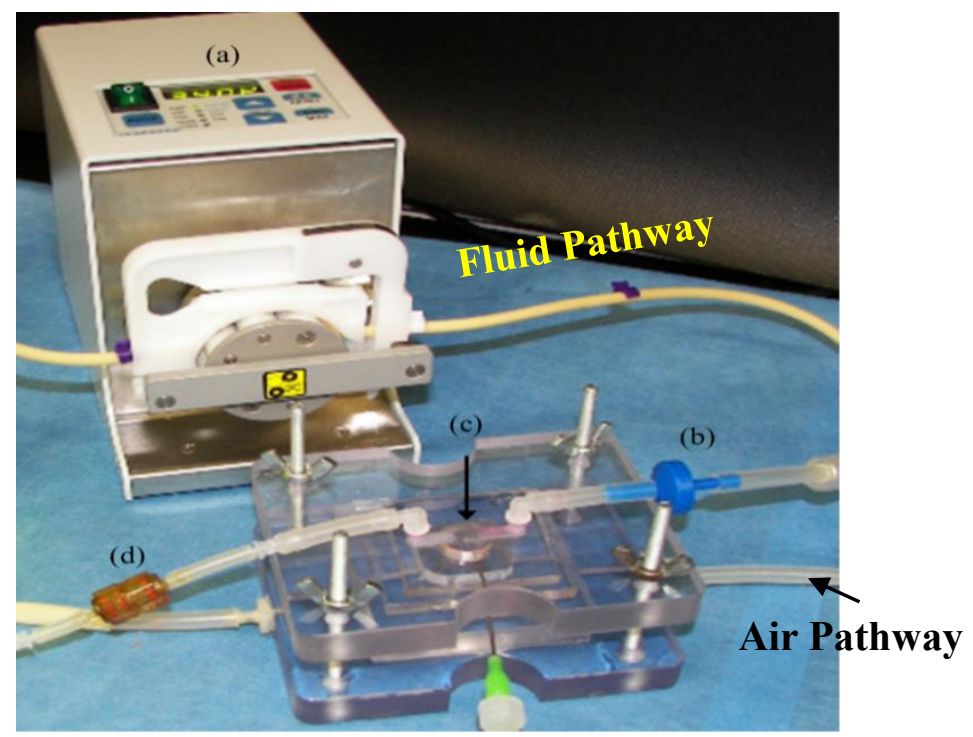

Figure 4.1: A schematic image of the second generation CCCM system. [A] 2 pathways are described: fluid pathway and air pressure pathway. [B] Picture of the CCCM system 
where (a) the peristaltic pump, (b) upstream one-way valve, (c) the CCCM platform with cell culture chamber (arrow) and (d) the downstream one- way valve.

The cell culture platform station includes a cell culture chamber, an oil chamber, and the housing station (Fig. 4.2). The cell culture and oil chambers are placed on top of each other and in between the top and bottom plates of the housing station. Cells are cultured on a thin flexible PDMS membrane, which forms the base of the cell culture chamber and is thus sandwiched between the cell culture and oil chambers. This cell culture membrane deforms as fluid is loaded and unloaded to the chamber, applying cyclic stretch and relaxation to the cells. Underneath of the oil chamber is a post membrane. Therefore, the cell culture system is a chamber-membrane-chamber-membrane stack (Fig. 4.2.C). The oil chamber transfers the displacement between the cell culture membrane and the post membrane (Fig. 4.2.E). The post membrane prevents the cell culture membrane from moving beyond its original position when the pneumatic pressure is applied on the other side of the post membrane (Fig. 4.2F). Finally, the housing station has top and bottom plates to sandwich the cell culture and oil chambers and to install the fluid and air networks. The top plate of the housing station has an inlet and outlet ports for fluid loaded and unloaded to the cell culture chamber while the bottom plate has the inlet and outlet ports for the air from the air pathway in and out of the station. 
(a)

(b)

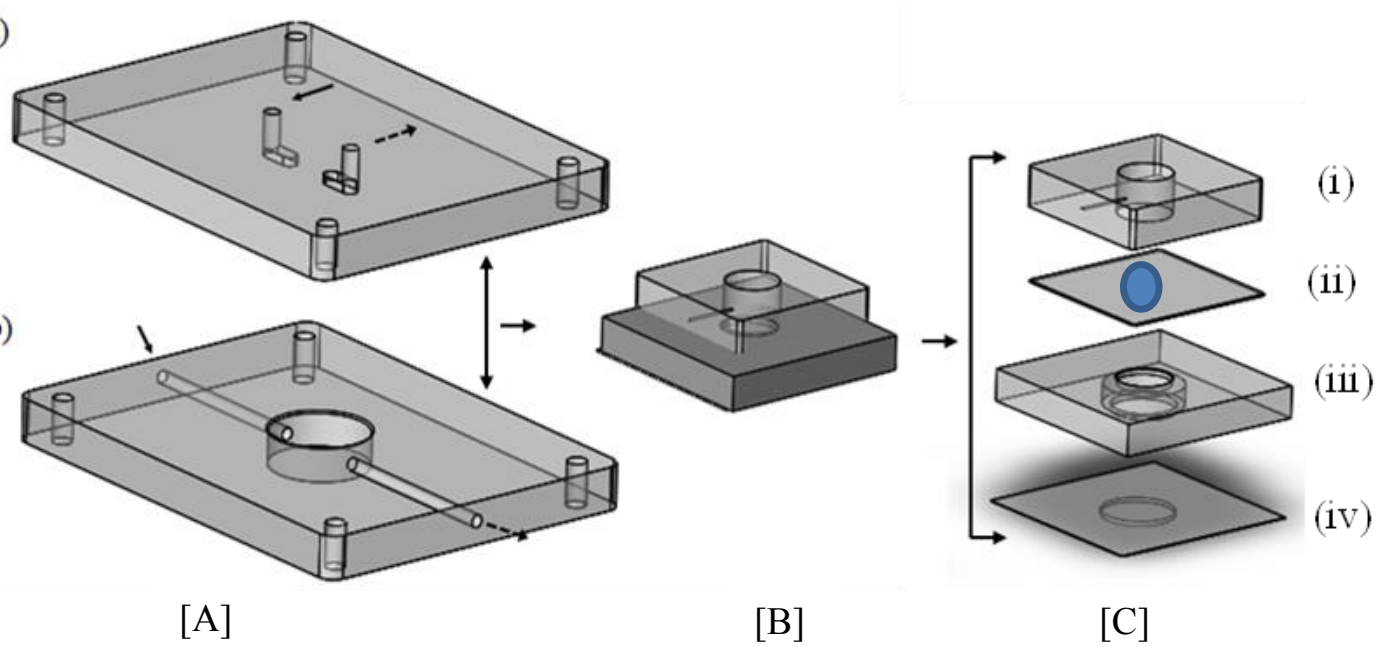

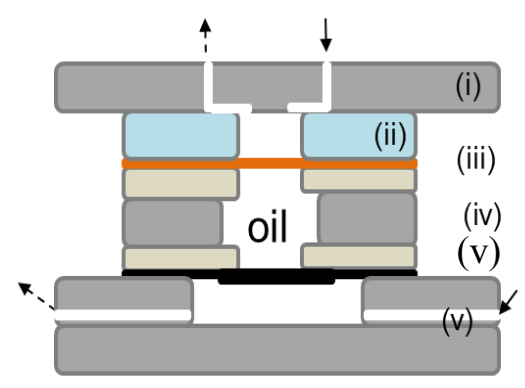

[D]

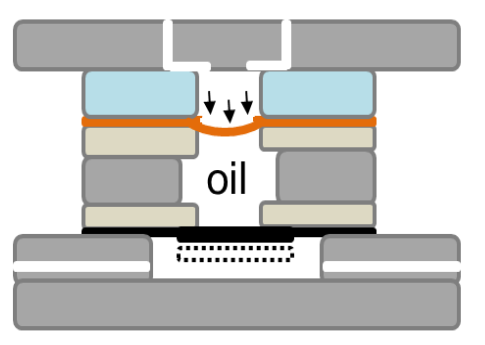

$[\mathrm{F}]$

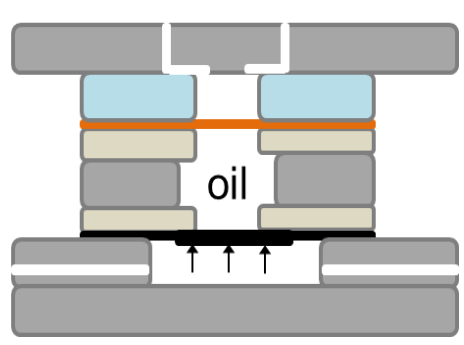

[F]

Figure 4.2: Scheme of the CCCM cell culture platform station. [A]: the housing station has (a) top plate with fluid inlet (solid arrow) and outlet (breakdown arrow) and (b) bottom plate with air inlet (solid arrow) and outlet (breakdown arrow). [B]: the assembled CCCM chambers. The assembled CCCM is sandwiched between plates of the station. $[\mathrm{C}]$ : the components of the assembled CCCM chambers: (i) cell culture chamber, (i) a flexible membrane where the cells are seeded (blue), (iii) oil chamber, and (iv) the post membrane. The assembled CCCM system is a chamber-membrane-chambermembrane stack. [D] Side view of the CCCM cell culture station: (i) top plate with fluid inlet (solid arrow) and outlet (breakdown arrow); (ii) cell culture chamber; (iii) cell membrane (orange); (iv) oil chamber made by 3 components (top, middle, and bottom)and filled with oil, and (v) the post membrane (black color) bottom plate with air 
inlet and outlet. [E] The scheme of membrane deformed when fluid is loaded to the cell chamber. This case, oil in the oil chamber transfers the displacement between the cell culture membrane and the post membrane, pushing the post membrane down (breakdown bar). [F] Cell membrane goes back to its original position when the pneumatic pressure is applied on the other side of the post membrane. Due to the block from the bottom component of the oil chamber, the post membrane cannot move up further than its original position, preventing the cell culture membrane from moving beyond its original position.

Fluid flows into the cell chamber, deforming the membrane downward. The pressure driver provides cyclic pressure on the other side of the post membrane, pushing the deformed polymer membrane back to its original position. Releasing this pressure stretches down the cell culture membrane during the fluid loading phase (Fig. 4.3). The frequency of this pressure as well as the percentage of loaded and unloaded time per each cycle is controlled by the program set in the pressure driver.

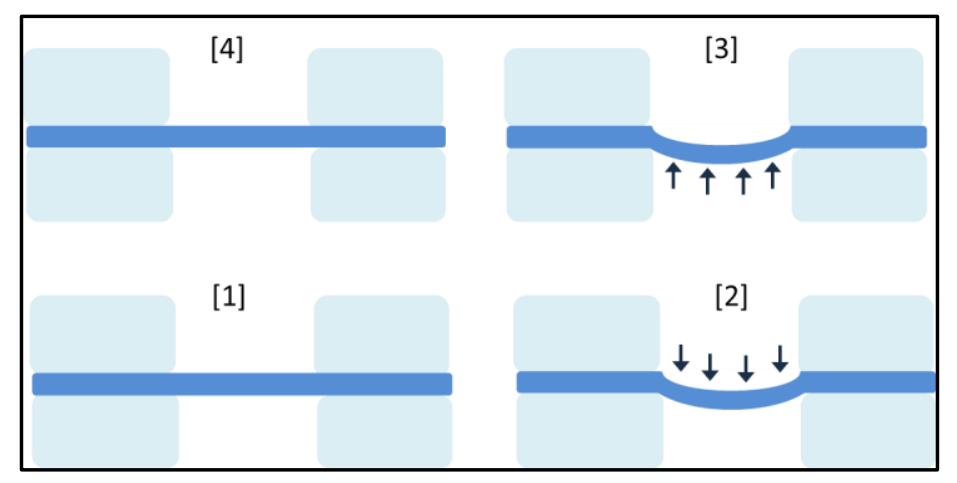

Figure 4.3: Scheme of the PDMS membrane deflection under the influence of fluid and pneumatic pressure loading and unloading. [1, lower left] PDMS membrane in the phase 
where no fluid filled into the cell culture chamber, the membrane has a horizontal position with no stretch. [2, lower right] the membrane deforms downward when fluid flows into the chamber from above (arrows down), [3, upper right] the membrane is pushed back to its original horizontal position when outside pressure applied to the inferior side of the post membrane, pushing the cell membrane up (arrows up); and [4, upper left] after ejection occurs the membrane is returned to its original position and one cycle of stretching-relaxing is completed.

The volume of the fluid loaded to the cell chamber is controlled by the peristaltic pump. Cyclic pressure inside the cell culture chamber is generated via the tunable pressure release valve and the pneumatic pressure inserted on the other side of the post membrane. The frequency and the air pressure loaded and unloaded to the station are controlled by the pressure generator. Finally, a pressure sensor connected to the cell culture chamber is monitored via real-time LabView program; and the pressure waveform inside the cell culture is hence recorded (Fig.4.4). 


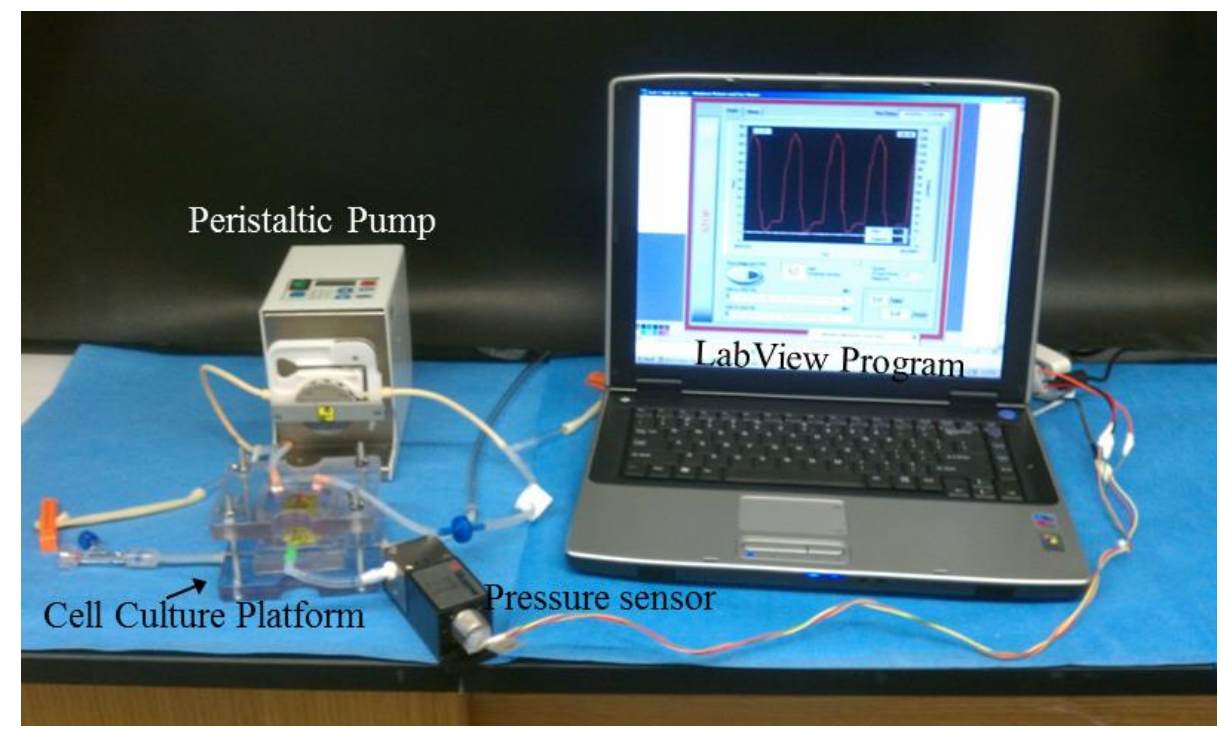

Figure 4.4: Image of the CCCM system connected to the LabView control system via pressure sensor.

\subsection{DESIGN AND FABRICATION OF DIFFERENT COMPONENTS OF THE CCCM SYSTEM}

\subsubsection{Design the Top and Bottom Plates of Cell Culture Housing Station}

The cell culture station was made in the Rapid Prototyping Facility at the University of Louisville using the Computer Numerical Control (CNC) Milling Machine. The designs were laid out using SolidWorks software prior to machining the assembly using CNC milling. Acrylic was the material used for construction.

This station consists of the top and bottom rectangular plates $(10.5 \mathrm{~cm} \times 9.0 \mathrm{~cm} \mathrm{x} 1.3 \mathrm{~cm})$ (Fig. 4.5). Two channels in the top plate serve as the entry and exit ports for fluid loading and unloading to the cell culture chamber. The distance between two inner ends of these channels is $0.6 \mathrm{~cm}$. The bottom plate of the platform has a circular hole $(\mathrm{d}=1.6$ $\mathrm{cm}, \mathrm{h}=1.0 \mathrm{~cm}$ ) at the center and 2 horizontal passages from the center hole to the outside 
edges $(1=4.6 \mathrm{~cm}, \mathrm{~d}=0.4 \mathrm{~cm})$, creating the inlet and outlet air channels. Finally, 4 holes were drilled at 4 corners of these components for screw insertion.

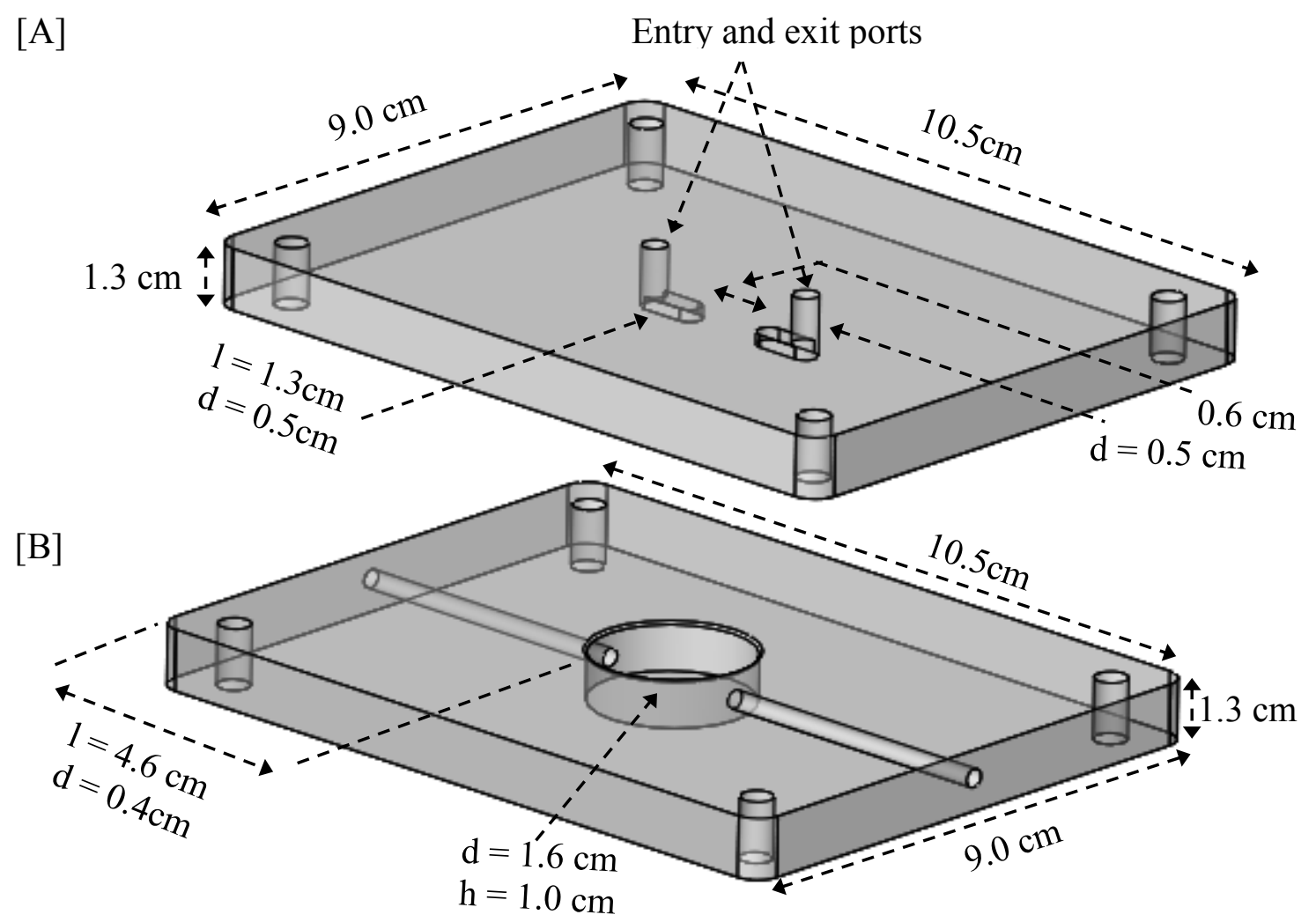

Figure 4.5: Schematic of the top and bottom plates of the station for housing the cell culture chamber. [A] the top plate of the platform with entry and exit ports for fluid loading and unloading; and [B] the bottom plate of the platform with a circular hole at the center and two horizontal passages for pneumatic pressure pathway.

\subsubsection{Fabrication of the thin PDMS membrane}

The thin PDMS membrane was made from Sylgard ${ }^{\circledR} 184$ polydimethyl siloxane (PDMS) (Dow Corning, Midland, MI) using standard soft-lithography techniques. First of all, a mixture of silicon elastomeric base with its curing agent (ratio 10:1) was well mixed, followed by a 15 minute vacuum to remove air bubbles created during the mixing 
process. To make a thin PDMS membrane less than $400 \mu \mathrm{m}$ thick, spinning method was used and the thickness of the membrane was determined by the spinning speed and time (Fig. 4.6). The degassed PDMS mixture was poured on a non-oxidized silicon wafer (0.525 mm thick, $100 \mathrm{~mm}$ diameter, Silicon Valley Microelectronics, Inc. Santa Clara, CA) and spun at a desire speed for $30 \mathrm{sec}$ on a Headway Spinner (Brewer Science, Inc., Rolla, MO). The PDMS coated wafer was then baked at $90^{\circ} \mathrm{C}$ in an oven (Fisher Isotemp, Florence, $\mathrm{KY}$ ) for at least $1 \mathrm{~h}$. Once the PDMS was cured, the wafer was removed from the incubator. This method was used to make the post membrane.

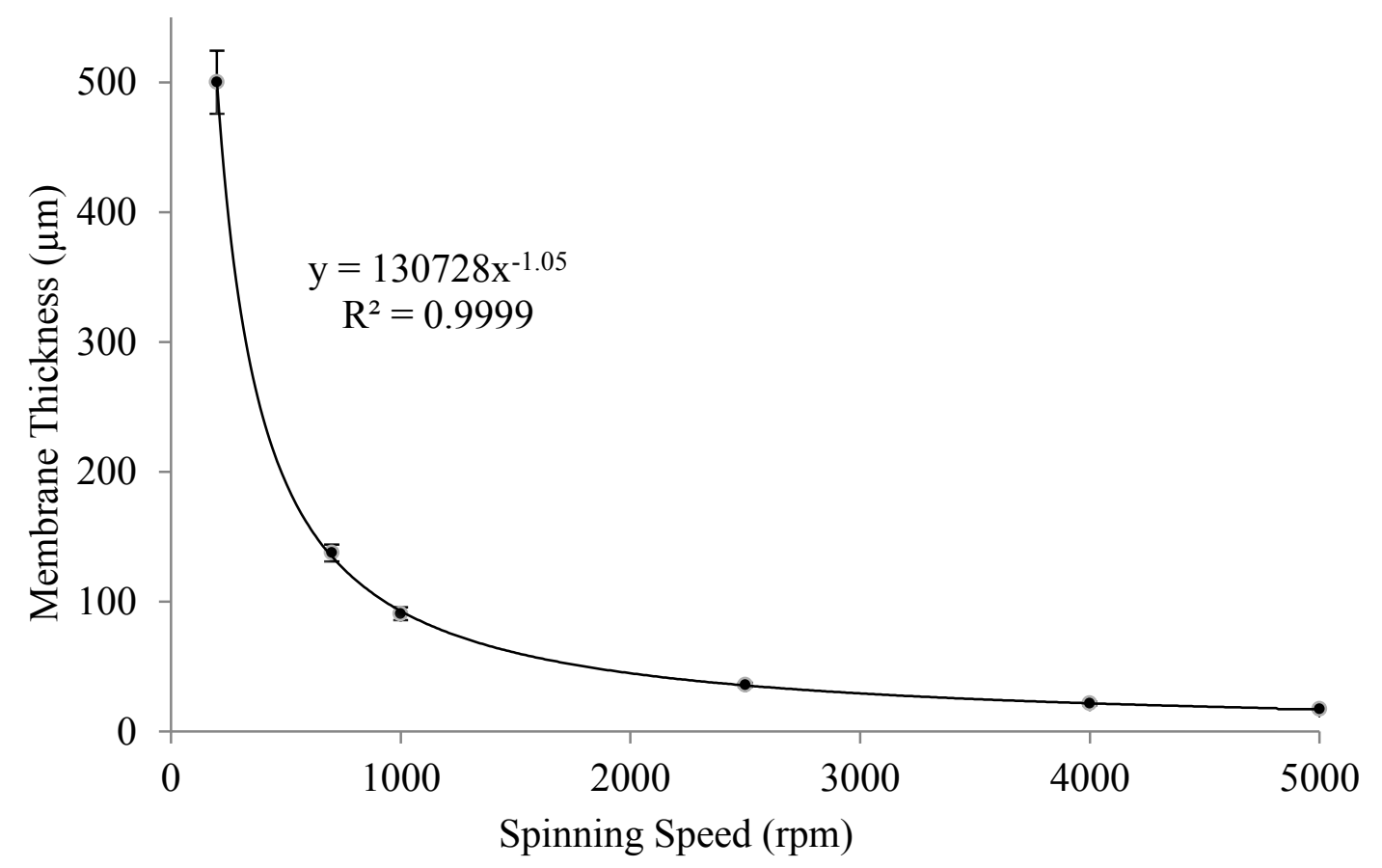

Figure 4.6: Graph of PDMS membrane thickness $(\mu \mathrm{m})$ versus spinning speed $(\mathrm{rpm})$. The spinning time is $30 \mathrm{sec}$. The membrane thickness as a function of spinning speed can be estimated using the equation $\mathrm{y}=130728 \mathrm{x}^{-1.05}$.

For the PDMS membrane thicker than $400 \mu \mathrm{m}$, gasket method was used. Basically, the degased PDMS was poured to a square gasket $(13.0 \mathrm{~cm}$ x $13.0 \mathrm{~cm}$ outside and $12.0 \mathrm{~cm} \times$ 
$12.0 \mathrm{~cm}$ inside) sitting on a square glass $(13.0 \mathrm{~cm} \times 13.0 \mathrm{~cm})$. The thickness of the gasket determines the membrane thickness. A thin transparent plastic sheet was then slowly placed on top of the PDMS mixture followed by another square glass. The whole setup was clamped tightly with paper clips and was put in the $90^{\circ} \mathrm{C}$ oven (Fisher Isotemp, Florence, KY) for being cured overnight to form a thin PDMS sheet (Fig. 4.7.). This method was applied to create the PDMS membrane at the bottom of cell culture chamber.

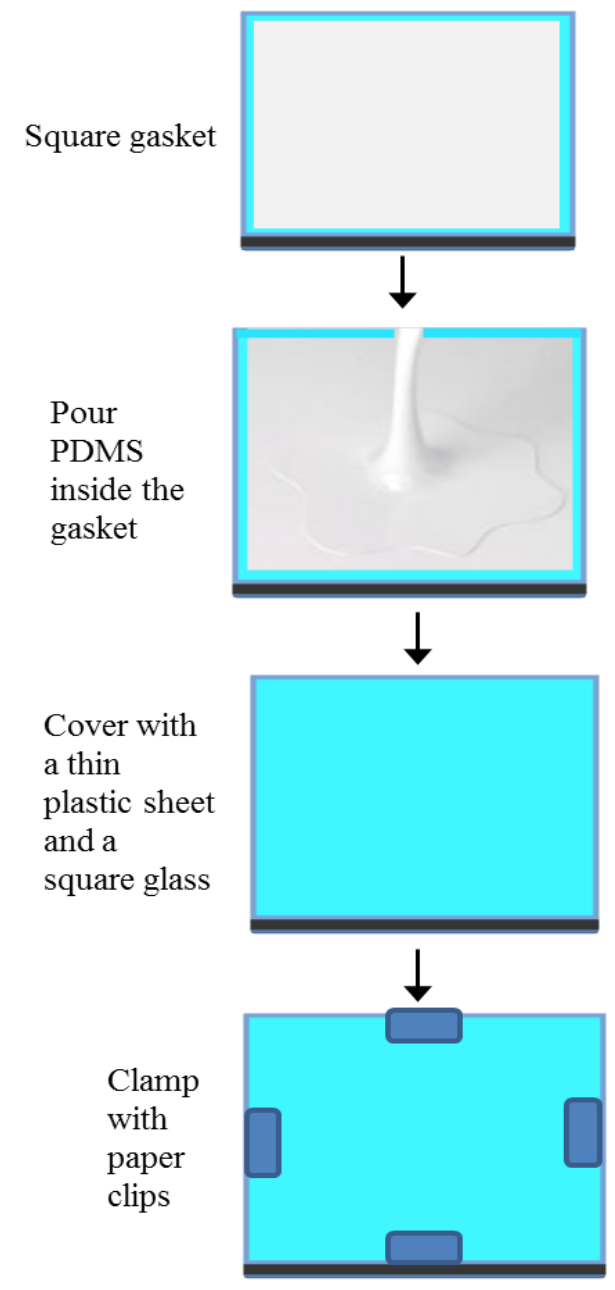

Figure 4.7: Fabrication steps of making a thin PDMS membrane via the gasket method. 


\subsubsection{Cell culture chamber and medium reservoir}

For the cell culture chamber, the degassed PDMS mixture (10:1 ratio) was poured in a mold until it reached $1.0 \mathrm{~cm}$ high. After being cured at $90^{\circ} \mathrm{C}$ in the oven, the molded PDMS was removed from the container and a square piece of PDMS $(3.0 \mathrm{~cm} \times 3.0 \mathrm{~cm} \times$ $1.0 \mathrm{~cm})$ was cut (Fig. 4.8). A hole $(\mathrm{d}=1.4 \mathrm{~cm})$ was punched at the center of this PDMS piece using a cork borer. Finally, a thin PDMS cell membrane, which was previously made, was placed at the bottom of this PDMS to form the cell culture chamber.

To provide medium for cells cultured before placing them to the CCCM system, a medium reservoir was also created. A hollow PDMS square piece $(2.5 \mathrm{~cm} \times 2.5 \mathrm{~cm}$ outside and $2.0 \mathrm{~cm} \times 2.0 \mathrm{~cm}$ inside) was cut from the molded PDMS (Fig.4.8). This reservoir was then placed on top of the cell culture chamber before sterilization.

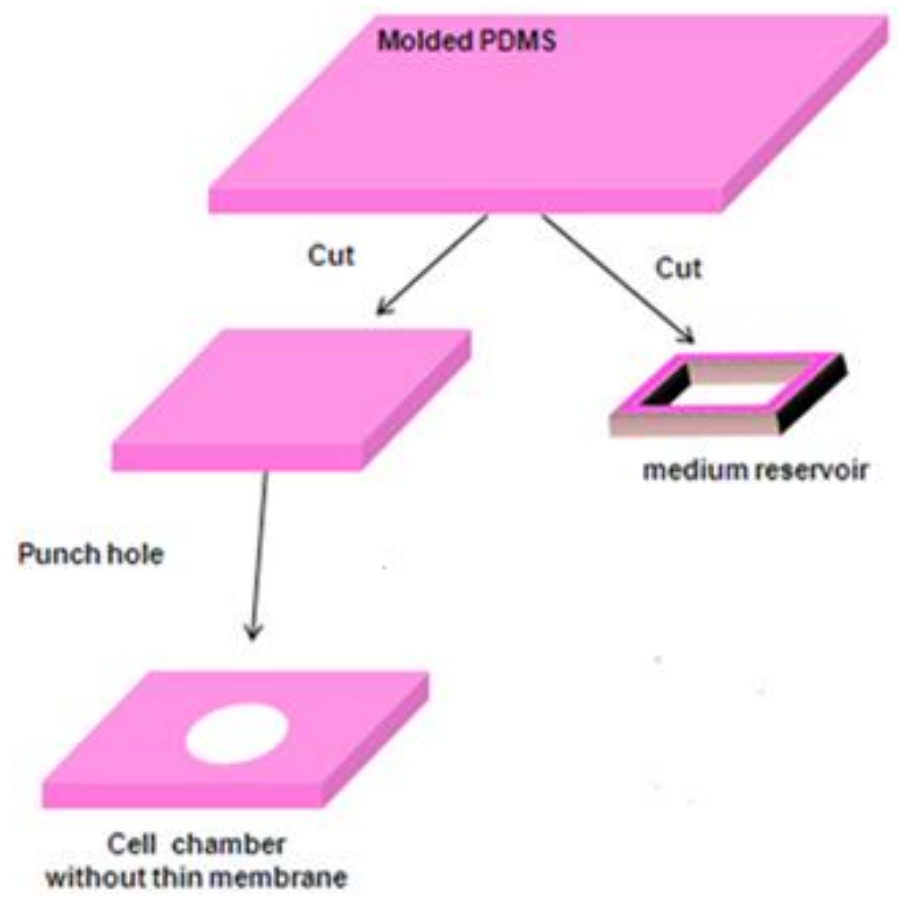

Figure 4.8: Fabrication steps involved in construction of the cell chamber and the medium reservoir. 
To measure the pressure inside the cell culture chamber, an accessible pressure chamber was created. The molded PDMS slack was cut $(3 \mathrm{~cm} \times 3 \mathrm{~cm})$ and a center hole was punched, similar to the cell culture chamber. A horizontal channel was then created from the center hole to the edge via an 18 gauge blunt syringe needle (SmallParts, NE181PLC). Finally, a 24 gauge blunt syringe needle (SmallParts) was inserted to this horizontal channel, served as a connecting port for the pressure sensor (Fig. 4.9.).

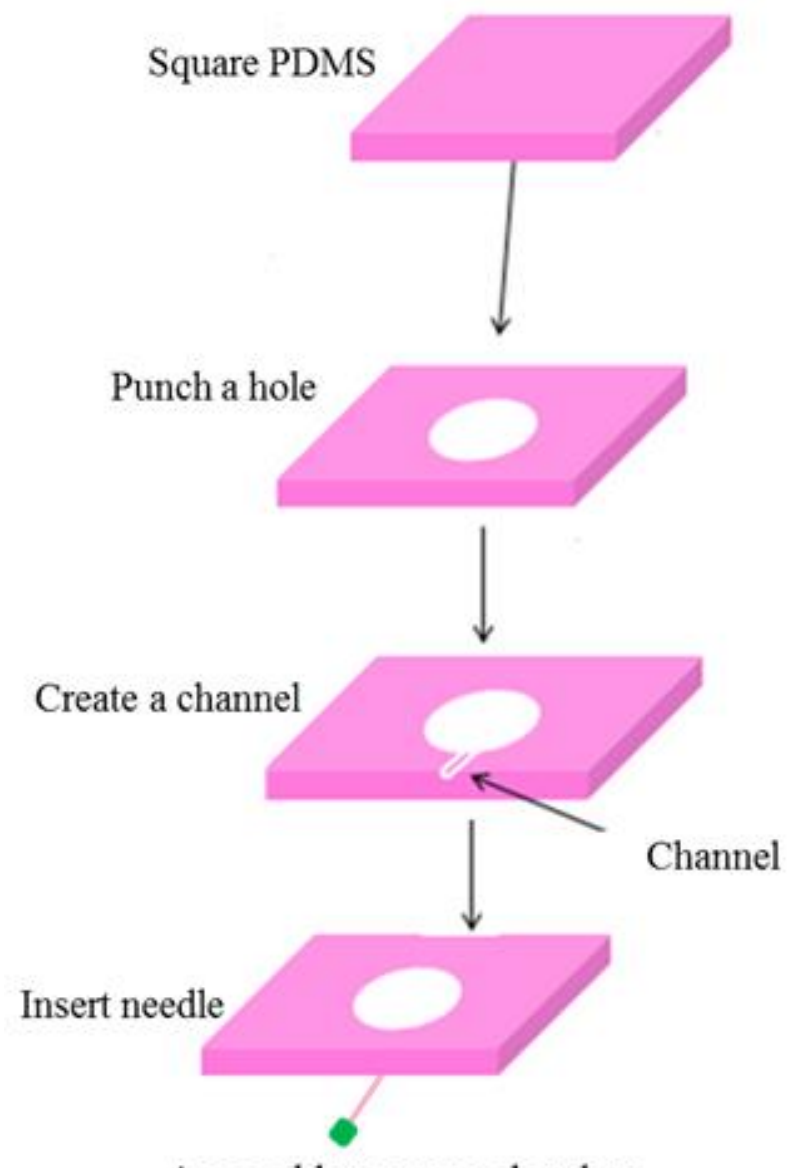

Assessable pressure chamber

Figure 4.9: Schematic representation of fabrication steps involved in construction of the assessable pressure chamber. 


\subsubsection{Oil chamber}

The oil chamber was built in a mechanical shop Facility at the University of Louisville. Three square acrylic pieces were cut and a circular hole was grilled at the center of each piece. The dimensions of each squares acrylic pieces were in the table 4.1 below. The edges of these pieces were smoothed before these pieces were glued together respectively to form the oil chamber. (Fig. 4.10, left)

Table 4.1: Dimensions of each component of the oil chamber

\begin{tabular}{|c|c|c|c|}
\hline Components & Thickness & Square dimensions & Hole diameter \\
\hline Top component (i) & $0.3 \mathrm{~cm}$ & $4.0 \mathrm{~cm} \mathrm{x} 4.0 \mathrm{~cm}$ & $1.3 \mathrm{~cm}$ \\
\hline Middle component (ii) & $1.0 \mathrm{~cm}$ & $4.0 \mathrm{~cm} \mathrm{x} 4.0 \mathrm{~cm}$ & $2.5 \mathrm{~cm}$ \\
\hline Bottom component (iii) & $0.3 \mathrm{~cm}$ & $4.0 \mathrm{~cm} \mathrm{x} 4.0 \mathrm{~cm}$ & $2.0 \mathrm{~cm}$ \\
\hline
\end{tabular}

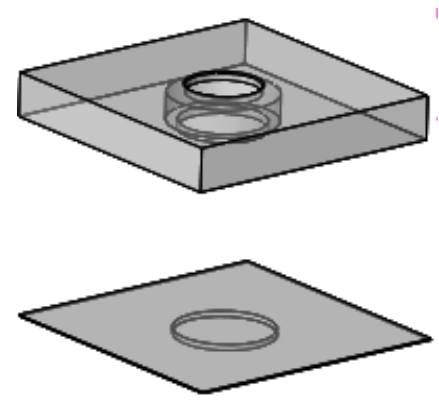

[A]

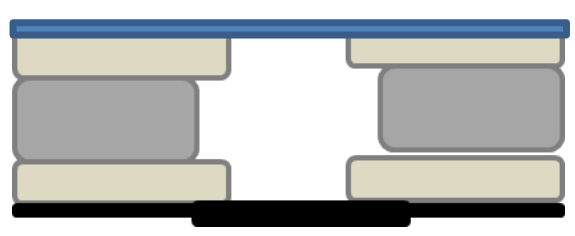

(i)

(ii)

\section{$[B]$}

Figure 4.10: Schematic images of the oil chamber. [A] The oil chamber (top) and the post membrane (bottom). [B] Side view of the oil chamber: top component (i), middle component (ii), bottom component (iii) and the post membrane (black). Oil chamber is filled with oil. PDMS cell culture membrane (dark blue) is placed directly on the top of the component (i). 


\subsubsection{Fabrication of the post membrane}

A thin $400 \mu \mathrm{m}$ PDMS (4.0 $\mathrm{cm} \times 4.0 \mathrm{~cm}$ ) previously made on a silicon wafer was peeled off. This thin PDMS membrane and a circular glass $(\mathrm{d}=2.5 \mathrm{~cm}, \mathrm{t}=0.25 \mathrm{~cm})$ were exposed to $10 \%$ oxygen plasma for $30 \mathrm{sec}$ in a reactive ion etcher system (RIE March Instruments) with $150 \mathrm{~mm}$ Torr and $150 \mathrm{mmHg}$. The membrane and circular glass were then bonded together centrically and irreversibly to form the post membrane (Fig.4.11.). Finally, the subject was heated at $115^{\circ} \mathrm{C}$ on a hotplate (Standard Model 1100 Hotplate, Brewer Science, Inc., Rolla, MO) for 10 min to enhance the quality of bonding.
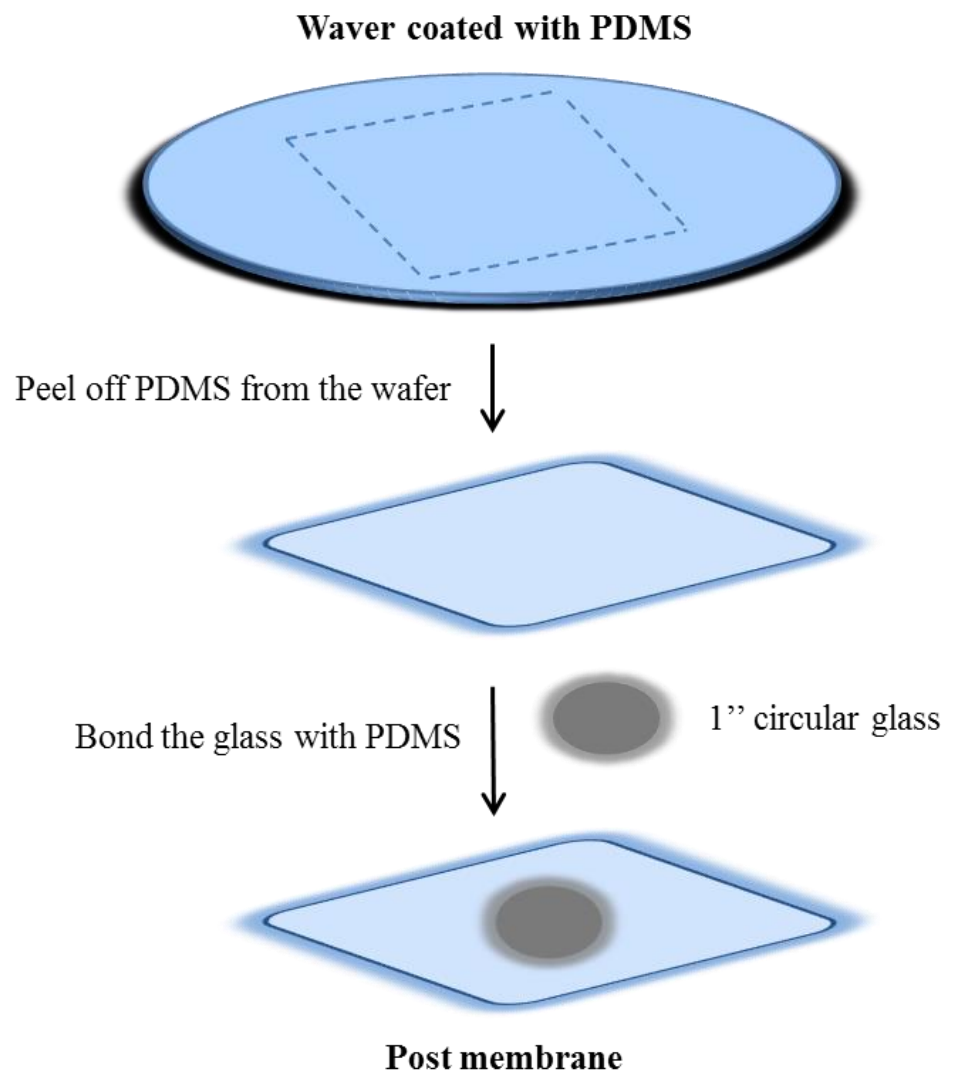

Figure 4.11: Schematic representation of fabrication steps involved in construction of the post membrane. 


\subsection{ASSEMBLY OF THE WHOLE CCCM SYSTEM}

\subsubsection{Assembly of the cell culture platform}

The cell culture platform was assembled in a sandwich style. In general, the post membrane, oil chamber, cell culture chamber, and pressure chamber were stacked on top of each other respectively and clamped between two fixtures of the apparatus. In detail, first of all, the oil chamber was firmly laid on the top of the post membrane to form a unit; and oil was used to fill this oil chamber. Secondly, after the reservoir on the top of cell chamber was removed, the accessible pressure chamber was placed on top of the cell culture chamber to form cell chamber unit. This unit was then placed centrically on top of the oil chamber without creating air bubble inside the oil chamber. Thirdly, these stacked membranes-chambers were placed on top of the bottom component followed by the top fixture. Finally, screws were used to tighten the components. (Fig. 4.12)

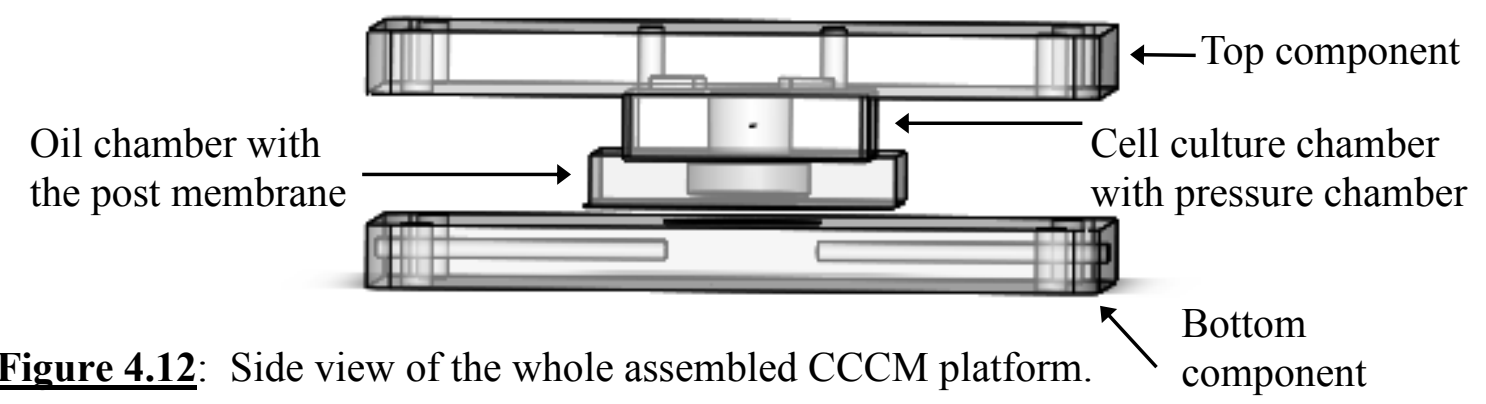

\subsubsection{Connection of the cell culture platform to the CCCM system}

The assembled cardiac culture platform was then connected to the flow loop and cyclic pressure network as shown on Fig. 4.2. First, a $50 \mathrm{~mL}$ tube served as the medium reservoir to supply fluid to the cell chamber and to receive fluid flowing back from the cell culture chamber via a peristaltic pump, ensuring a continuous supply. Fluid compliance and a one-way valve were connected to the upstream of the fluid flow 
network. A one-way downstream valve, tunable valve, and a by-pass pathway with a pressure gate valve were installed at the down-stream of this flow loop. Flexible, autoclave compatible polymeric tubing was used to establish connections within the flow loop (Fig.4.13). Second, the pressure sensor was attached to the cell culture chamber via the needle port of the pressure chamber. Third, the air compliance was attached to the apparatus.

This whole set up was then moved to the incubator before the pneumatic pathway was connected to the platform. Once in the incubator, the air compliance was connected to downstream of the pneumatic pathway, while upstream of the airway was hooded to a pulsatile pneumatic pump (lb Engineering GbR). Finally, the pressure sensor was connected to the computer; and the pressure inside the cell culture chamber was transferred digitally to the LabView Program on the computer.

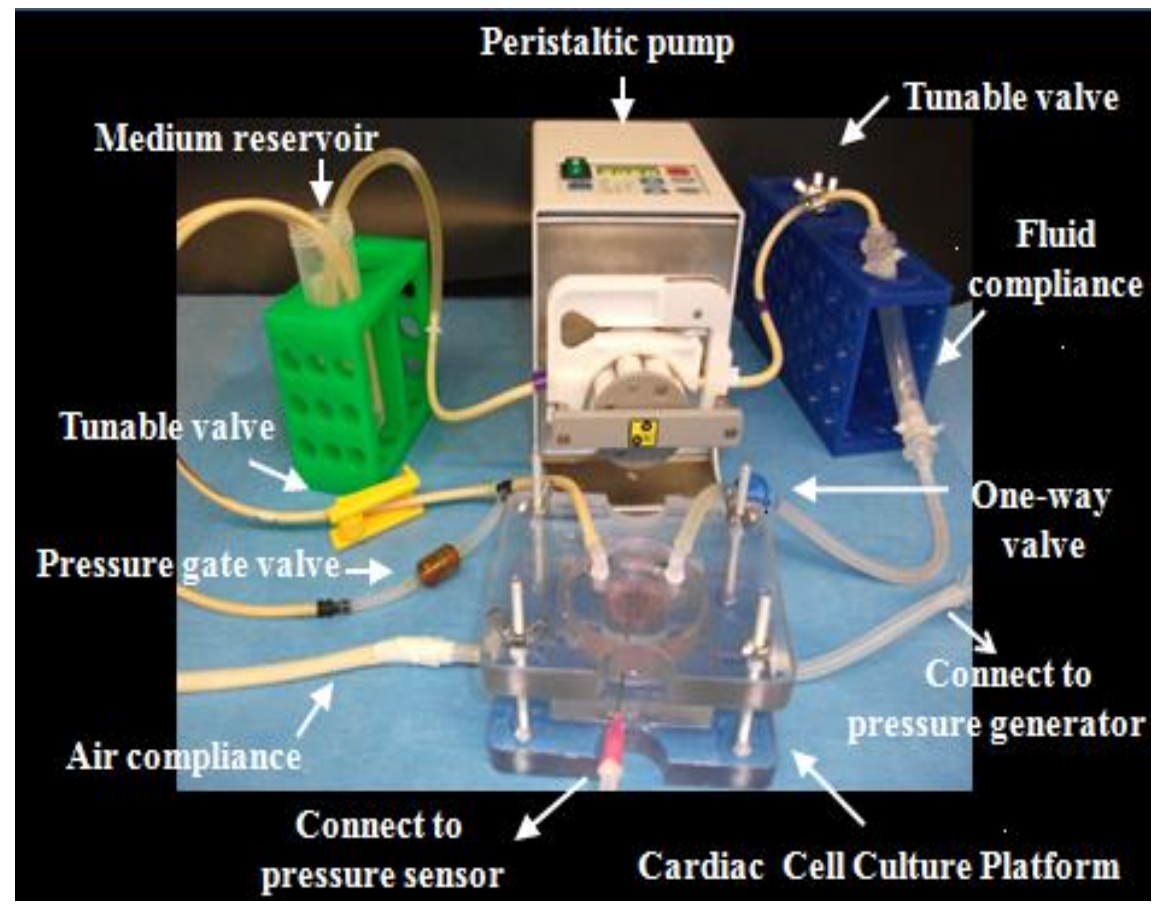

Figure 4.13: Picture of the CCCM experimental setup. 


\subsection{WORKING PRINCIPLES OF THE CCCM SYSTEM}

The working cycle of the CCCM mimics the working cycle of the left ventricle (Fig. 4.14). In the filling phase, fluid flow is introduced continuously into the CCCM cell culture chamber via the peristaltic pump. Fluid fills the cell culture chamber causing the PDMS membrane to stretch passively. The stretching of the membrane maintains the pressure inside the cell culture chamber as low as the base pressure should be. This stretching phase mimics the left ventricular diastolic filling. During the isovolumic contracting phase, air pressure generated by the pulsatile pneumatic pump (the frequency and pressure are programed based on each specific experiment) is applied to the reverse side of the post membrane, pushing the PDMS cell membrane back to its original position. Pressure is thus built up rapidly within the cell culture chamber, causing the upstream one-way valve to close. Because of this valve closure, the fluid from the peristaltic pump begins to be stored in the fluid compliance. However, the cell chamber pressure at this point is not high enough to overcome the downstream resistance, therefore, no fluid is loaded or unloaded to the chamber. This phase is similar to the isometric contracting phase inside the left ventricle. For the ejecting phase, the pressure inside the cell chamber increasing until it exceeds the set value of the resistance to open the downstream valve and then the accumulated fluid loaded in the cell culture is ejected. Finally, the pressure applied to the reverse side of the membrane is released, causing the CCCM to undergo isovolumic relaxation before another cycle begins. This phase mimics the isometric relaxing phase of the left ventricle. 

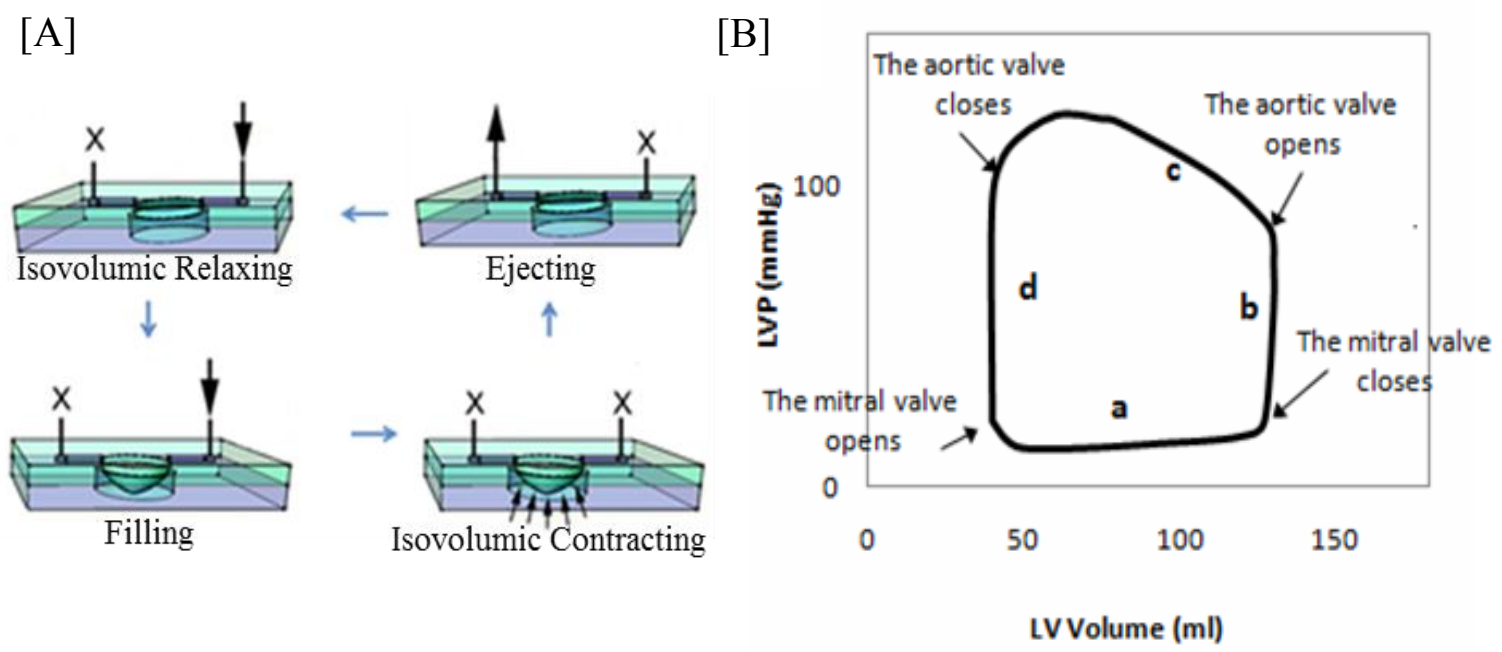

Figure 4.14: A working cycle of the CCCM system and the pressure-volume loop occurred in left ventricle of the heart. [A] Schematic working principles of the CCCM system, including stretching phase (fluid loading causes the membrane stretched down), contracting phase (pneumatic pressure applied to the other side of the membrane brings the membrane back to the original position and elevates the chamber pressure), ejecting phase (chamber pressure overcomes the downstream resistance to eject the fluid out of the chamber), and the relaxing phase (no fluid in or out). X: valve is closed; 4 : fluid direction. [B] Pressure-Volume loop seen in the left ventricle: (a) diastolic filling phase, (b) isovolumic contraction phase, (c) ejecting phase, and (d) isovolumic relaxation phase.

Additionally, a programmable pressure generator is used to supply periodic pulses of pressurized air to the reverse side of the polymeric membrane on which cells are cultured to mimic ventricular contraction. Finally two tunable compliance elements, a fluidic compliance integrated prior to the upstream one-way valve, and air compliance element integrated at the end of the pressure generator flow path, are used to modulate pressure waveforms within the cell culture chamber. Therefore, with the pulsatile pneumatic 
pump, the frequency of the stretching-contracting of the PDMS cell culture membrane is controlled; and the cylclic pressure inside the cell culture membrane is also generated, similar to the cyclic pressure seen in the left ventricle.

\subsection{OPERATION OF THE CCCM SYSTEM}

The assembly of the CCCM system was performed inside the cell culture hood. Once the CCCM platform station and the fluid network were assembled as in Fig. 4.13, the whole set up system was moved into the cell incubator. Then, the peristaltic pump began to run, followed by the connection of the air network to the pressure generator, which had been on. Along with the controlling from the pressure generator, the downstream tunable valve was adjusted until the pressure reached the desirable values. Variable parameters, such as the frequency of the membrane stretching as well as the percentage of systolic phase per one cycle, were determined based on the requirement of each experiment. The frequency of the stretching was controlled through the systolic time and the frequency control on the pressure generator source. The pressure inside the cell chamber was varied depending on the pressure applied on the post membrane and the adjustment of the tunable valve. At the end of the experiment, peristaltic pump was turned off first followed by the pressure generator. Finally, the cell chamber was removed from the platform.

\subsection{CONCLUSION}

Recognizing the limitations of the $1^{\text {st }}$ generation $\mu \mathrm{CCCM}$ model, the second generation CCCM system was created to more accurately reproduce the mechanical loading and 
unloading conditions observed in the heart. This system is capable of accurately mimicking preload and afterload experienced in the healthy and failing heart. The CCCM allows for dynamic studies, accomplished via direct manipulation of flow rate, valve operation, membrane thickness and the height of the cell culture chamber. When working with different types of cardiac cells or different physiological heart conditions, it is important to ensure that the type of mechanical loads delivered such as the stress, stretching, frequency of stretching, and the shear stress can be dynamically controlled. The following chapter therefore focusses on the characterization of this CCCM system. 


\section{CHAPTER 5 \\ CHARACTERIZATION OF THE CCCM SYSTEM}

\subsection{YOUNG'S MODULUS OF THE THIN PDMS MEMBRANE}

\subsubsection{Introduction of PDMS}

Polydimethyl siloxane (PDMS) is a common silicon rubber used for various applications such as mechanical interconnective layers between 2 wafers, mechanical-chemical sensors, microvalves in fluid applications, and biomedical applications. ${ }^{123-125}$ PDMS Sylgard 184 from Dow Corning Corporation (Midland, MI) is one of the two most common silicone products. It contains a base polymer and curing agent. Cross-linking reaction is the main reaction for the curing of PDMS; and this curing process is enhanced via heating process. ${ }^{126}$ Therefore, the PDMS rigidity can be controlled by the ratio between its base and cross-linking agent, the heating temperature and the time of baking.

PDMS is a substrate widely used for cell behavior studies due to several advantages. PDMS is biocompatible, transparent, gas permeable, autoclavable, and elastic. This silicone is also physically and chemically stable. ${ }^{127}$ It has a high flexibility and compressibility, suitable to use for making a thin stretchable membrane in the CCCM system. The fabrication of PDMS membrane is straight forward, less time consumed, and cost effective, making it suitable for replication. ${ }^{125}$ PDMS is also hydrophobic; and yet, via oxygen plasma treatment, it becomes hydrophilic for a few minutes before going 
back to its original hydrophobic state. ${ }^{126}$ This special chemical characteristic of PDMS helps different PDMS layers bond to themselves and therefore creating a watertight seal between layers of the CCCM culture chamber when the PDMS becomes hydrophobic.

In this project, Young's modulus and strain value of the thin PDMS sheet used in the CCCM system were determined. Due to the fact that this thin PDMS membrane is an elastomer and has the ability to hold a large amount of strain, true stress $\sigma^{*}$ and true strain $\varepsilon^{*}$ were determined from the engineering stress $\sigma$ and strain $\varepsilon$ of the PDMS membrane. Young's modulus E of the thin PDMS membrane under the tensile force was obtained by the following equations: ${ }^{127,}, 128$

$$
\begin{gathered}
\sigma=\frac{F}{A_{0}} \quad \text { and } \quad \varepsilon=\frac{\Delta L}{L_{0}} \\
\sigma^{*}=\sigma(1+\varepsilon) \quad \text { and } \quad \varepsilon^{*}=\ln (1+\varepsilon) \\
E=\frac{\sigma^{*}}{\varepsilon^{*}}
\end{gathered}
$$

where $\mathrm{F}$ is the tensile force applied onto the membrane, $\mathrm{A}_{0}$ is the original cross-sectional area, $\Delta \mathrm{L}$ is the elongation of the membrane after stretched, and $\mathrm{L}_{0}$ is the original length of the membrane.

\subsubsection{Tensile test}

The RSA III Rheumatics System Analyzer was used for the PDMS tensile tests. First, the fabricating method for the thin PDMS sheet was described from section 4.2. The ratio of mixing between the base and curing agent was 10:1. The curing temperature was set at $90^{\circ} \mathrm{C}$ and the baking time was from $12 \mathrm{~h}$ or overnight. After cured, this thin PDMS 
sheet was cut into strips ( 0.5 inch $x 4.0$ inches) and vertically mounted in the way that one end of the strip was attached to a fixed fixture while the other end was clamped to a movable fixture of the RSA Analyzer (Fig. 5.1). Zero force was initialized and the tension force was applied. The membrane was pulled slowly with the rate of $0.5 \mathrm{~mm} / \mathrm{s}$ until it reached a $50 \%$ displacement ( 2 inches). Three different thicknesses of PDMS membranes $(250 \mu \mathrm{m}, 400 \mu \mathrm{m}$, and $500 \mu \mathrm{m})$ were used. At least five different PDMS pieces molded on different days with the same thickness were made for this tensile test. The excel program was used to determine the stress, strain, and Young's modulus of the PDMS.
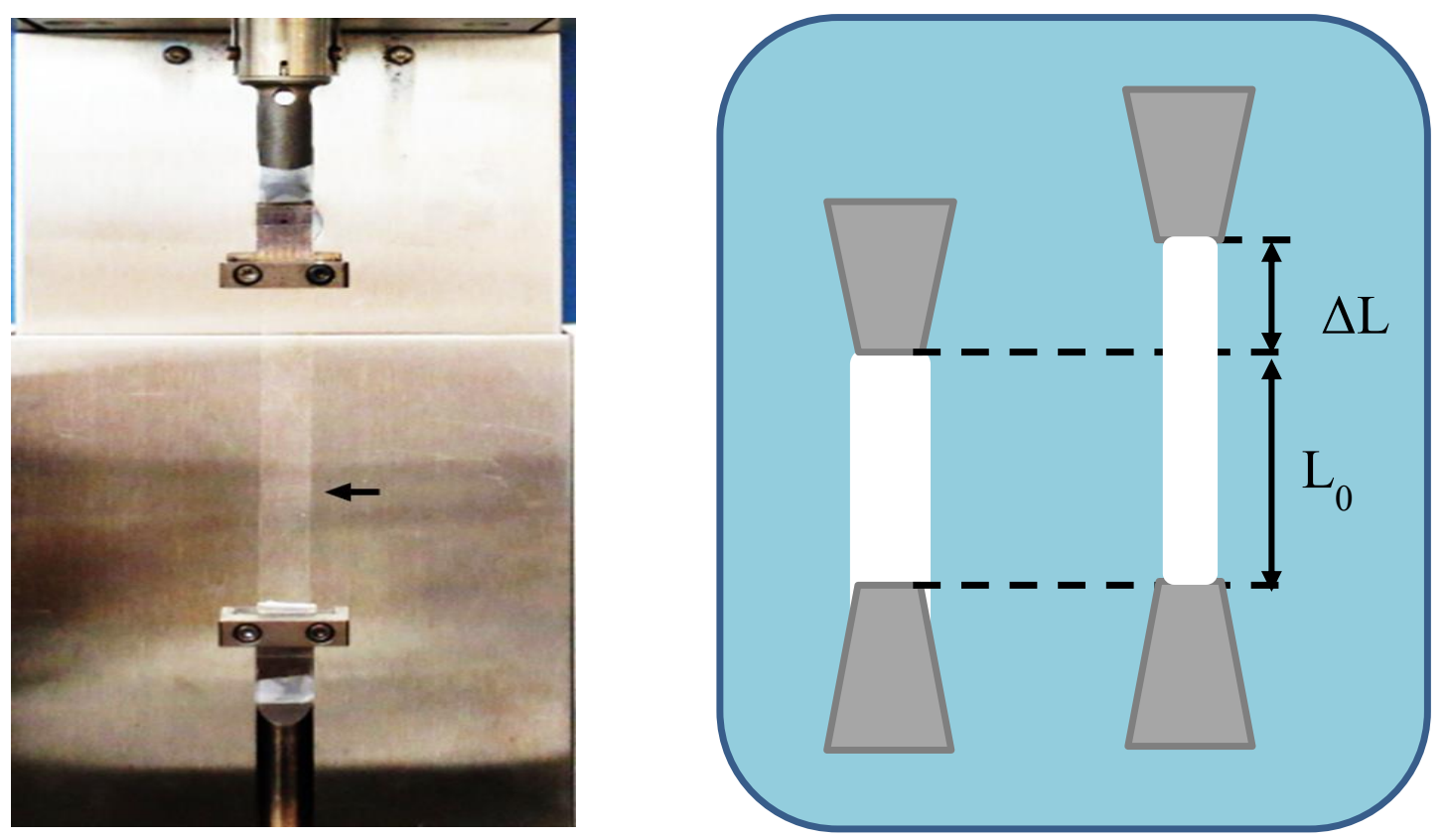

Figure 5.1: Experimental setup image for the strain and Young's Modulus measurement of PDMS membranes. Left: The PDMS strip (arrow) was installed between 2 fixtures of the RSA Analyzer. Right: Sketch of the displacement of a thin PDMS strip. 


\subsubsection{Results and Discussion}

The mechanical properties and Young's modulus of different PDMS thicknesses were determined. Young's modulus of the PDMS depends greatly on the stress-strain curve, mixing ratio and strain rate. ${ }^{127}$ For consistency, the mixing ratio of 10:1 (base: curing agent) was carried throughout this project. Tensile tests on the PDMS membrane were conducted. During the testing process, the elongation of the strips and the tensile forces were recorded automatically by the system. By loading the dimensions of the PDMS strip to the system, the engineering stress and the engineering strain were obtained. Since the PDMS is an elastomer and has a capacity to hold a large amount of strain, the true stress and true strain derived from engineering strain were used to calculate the elastic modulus of the PDMS (Fig. 5.2.) The results shows that with the PDMS thickness from $250 \mu \mathrm{m}$ to $500 \mu \mathrm{m}$, Young's modulus and stress-strain relationship were not significantly different among each membrane thickness. The Young's modulus of the thin PDMS membrane was calculated using the equation (3) in the section 5.1.1, which was around $1.0 \pm 0.17 \mathrm{MPa}$. The graph of stress vs strain and Young's modulus value agree with the results obtained from other research groups. ${ }^{126-128}$ These results were helpful to assist the fabrication of various membranes with different thicknesses for cardiac culture. Amongst these three membrane thicknesses, membrane with the thickness of $500 \mu \mathrm{m}$ was used for cardiac tissue cultured in the CCCM system later. 

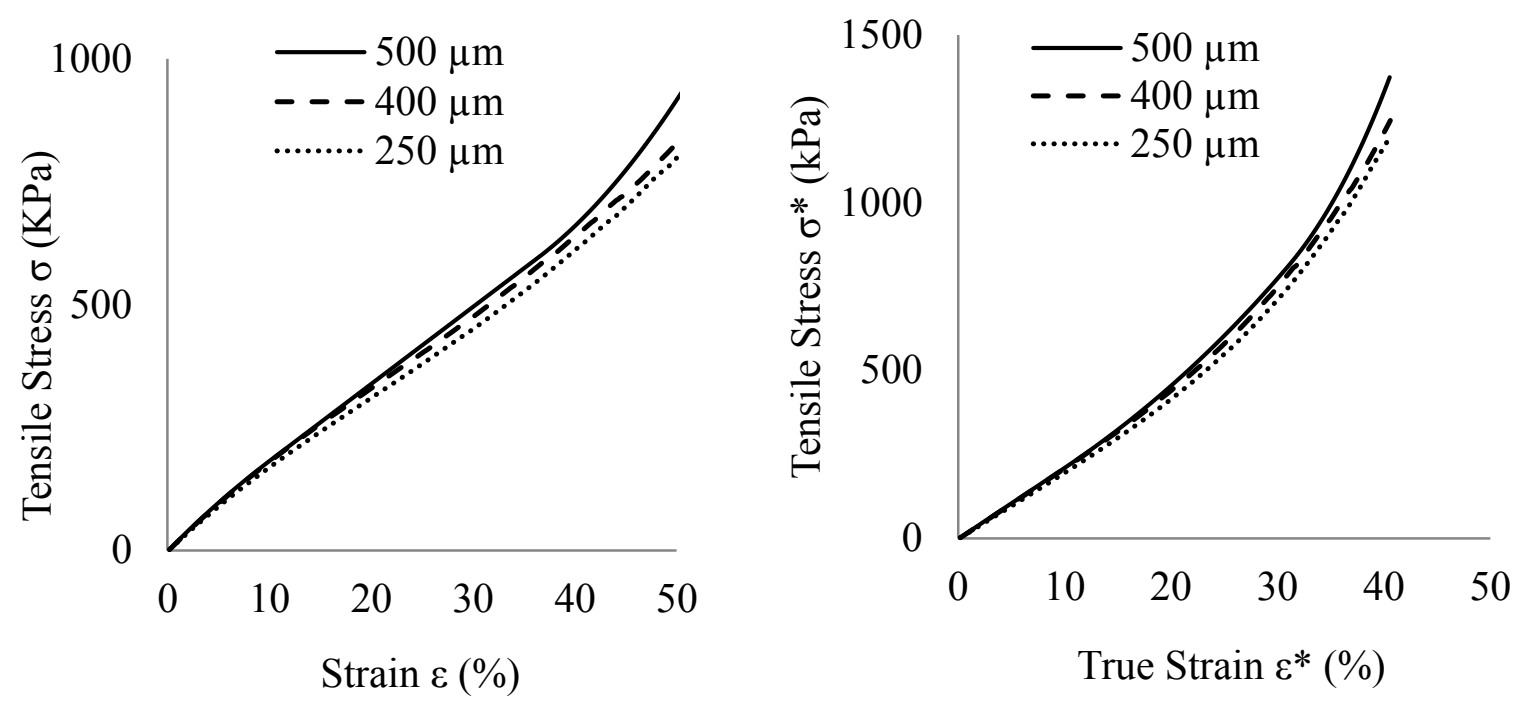

Figure 5.2: Graphs of stress versus strain of thin PDMS sheets

\subsection{PRESSURE GENERATED INSIDE THE CCCM CELL CHAMBER}

A synergistic relationship exists between the pump flow rate, membrane thickness, downstream resistances and the magnitude of pressure pulses within the CCCM. All these parameters need to be set precisely to ensure proper filling and ejection of fluid from the cell culture chamber and mechanical stresses. The pressure waveform generated from the CCCM cell culture chamber was determined by the pressure input from the pulsatile pneumatic pump, frequency, flow, percentage between the stretching and contracting phases of each cycle as well as the downstream resistance. These variable parameters were determined and adjusted based on the requirement of each experiment. The frequency of the stretching was controlled by the frequency set up on the pressure generator. The pressure inside the cell chamber was mainly varied by the cyclic pressure applied on the post membrane and the adjustment of the tunable valve. For the proof of concept, the profile of pressure wave forms for different human heart conditions (normal, 
hypertension, hypotension, heart failure, bradycardia, and tachycardia ${ }^{111}$ seen in the left ventricle of human heart were generated inside the cell culture chamber of the CCCM system. (Table 5.1 and Fig.5.3.) This accomplishment indicates that this CCCM system can be a benefit for in-vitro cardiomyocyte studies under the effect of physiological mechanical relevance of different heart conditions.

Table 5.1: Hemodynamic parameters corresponding to the left ventricular mechanical loading during various clinical conditions were replicated using the CCCM system. These parameters include heart rate, systole as percent of the cardiac cycle, end systolic pressure, and end diastolic pressure of the left ventricle were applied to the CCCM system to obtain the pressure waveforms in the following figure 5.3 below. LV (left ventricle).

\begin{tabular}{|ccccc|}
\hline Conditions & $\begin{array}{c}\text { Heart Rate } \\
\text { (bpm) }\end{array}$ & $\begin{array}{c}\text { Systolic } \\
\text { Fraction } \\
(\%)\end{array}$ & $\begin{array}{c}\text { LV systolic } \\
\text { pressure } \\
(\mathbf{m m H g})\end{array}$ & $\begin{array}{c}\text { LV end diastolic } \\
\text { pressure } \\
(\mathbf{m m H g})\end{array}$ \\
Normal & 75 & 40 & 125 & 9 \\
Hypertension & 75 & 40 & 183 & 6 \\
Hypotension & 75 & 40 & 95 & 6 \\
Heart Failure & 75 & 40 & 100 & 27 \\
Tachycardia & 200 & 50 & 120 & 4 \\
Bradycardia & 46 & 40 & 124 & 8 \\
\hline
\end{tabular}



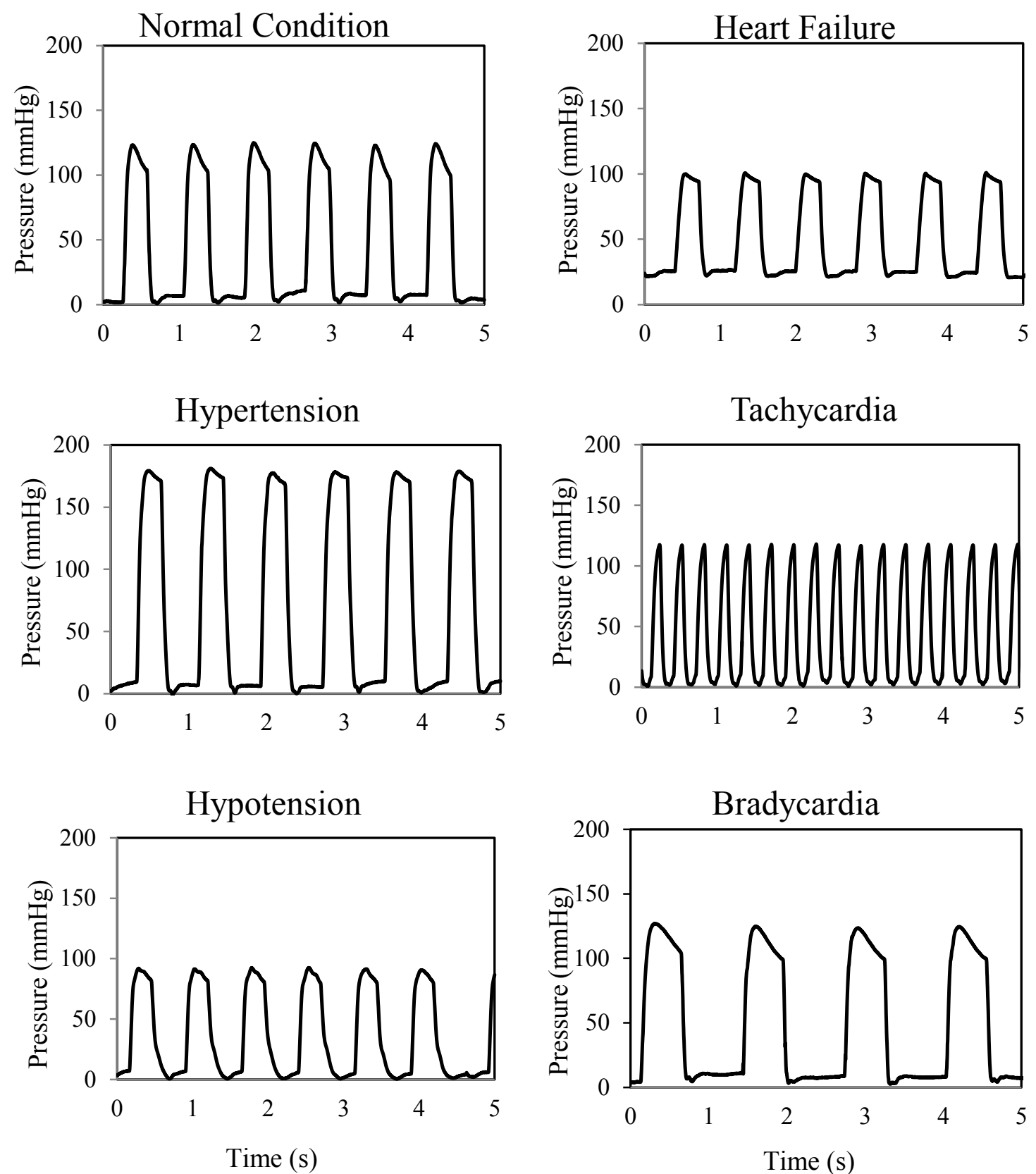

Figure 5.3: Pressure vs time plots representing the changes in pressure within the CCCM cell culture chamber for different human heart conditions. The parameter of the pressure and frequency were determined based on different conditions seen in the left ventricle of the human heart. 


\subsection{STRAIN OF THE PDMS CELL CULTURE MEMBRANE}

As the cell type changed from $\mathrm{H} 9 \mathrm{C} 2$ cells to embryonic chick ventricular cardiomyocytes as the primary cell line for characterization of the CCCM chamber, we set the CCCM strain to replicate the strain of the in-vivo chick embryonic left ventricle cardiomyocytes at stage of 36 ; which is reported to range from 0.10 to $0.20 .{ }^{129,130}$ For in-vitro CCCM culturing, strains of $8-15 \%$ were applied.

When fluid was induced to the cell culture chamber, PDMS experienced both radial and circumferential stretches (Fig. 5.4). These stretches of the PDMS membrane were also conveyed to the cells seeded on that membrane. Both strains were determined via the simulation from the ANSYS software program as well as via experimental membrane deflecting movies obtain from a Ultra Sound system.

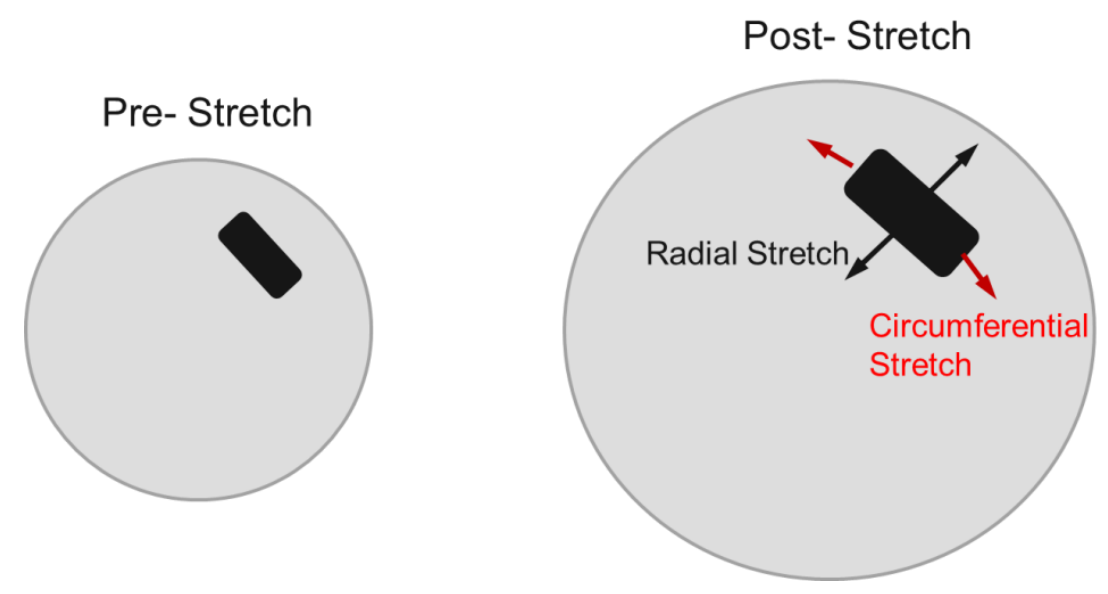

Figure 5.4: Scheme for the stretches of the circular membrane

\subsubsection{Membrane Deflection Strain measurement via ANSYS program}

The following formulas were used for calculation: 
○ Mooney- Rivlin strain energy (5 parameters) for hyper-elastic material (PDMS)

$$
\begin{aligned}
& \Psi=\mathrm{C}_{10}\left(\mathrm{~T}_{1}-3\right)+\mathrm{C}_{01}\left(\mathrm{~T}_{2}-3\right)+\mathrm{C}_{11}\left(\mathrm{~T}_{1}-3\right)\left(\mathrm{T}_{2}-3\right)+\mathrm{C}_{20}\left(\mathrm{~T}_{1}-3\right)^{2}+\mathrm{C}_{02}\left(\mathrm{~T}_{2}-3\right)^{2}+(1 / \mathrm{d})(\mathrm{J}-1)^{2} \\
& \mathrm{C}_{10}, \mathrm{C}_{01}, \mathrm{C}_{20}, \mathrm{C}_{11}, \mathrm{C}_{02}=\text { material constants } \\
& \mathrm{d}=\text { material incompressibility parameter } \\
& \mathrm{T}_{1}, \mathrm{~T}_{2}, \mathrm{~T}_{3}=\text { invariants of the right Cauchy-Green deformation tensor }
\end{aligned}
$$

○ Volume:

$$
V=\frac{1}{6} \pi h\left(3 R^{2}+h^{2}\right)
$$

V: volume; R: radius; and h: height

○ Radial strain $\epsilon_{\mathrm{r}}$ :

$$
\epsilon_{\mathrm{r}}=\frac{\mathrm{R}_{\mathrm{f}}-\mathrm{R}_{\mathrm{i}}}{\mathrm{R}_{\mathrm{i}}}
$$

$\mathrm{R}_{\mathrm{f}}$ and $\mathrm{R}_{\mathrm{i}}$ : final and initial radius of the membrane (Fig. 5.5)

○ Circumferential strain $\epsilon_{\mathrm{c}}$ (Green strain equation):

$$
\begin{aligned}
& \epsilon_{\mathrm{c}}=0.5\left(\lambda_{\mathrm{c}}^{2}-1\right) \\
& \lambda_{\mathrm{c}}=\left(2 \pi \mathrm{r}_{\mathrm{f}}\right) /\left(2 \pi \mathrm{r}_{\mathrm{i}}\right)
\end{aligned}
$$

$\lambda_{\mathrm{c}:}$ circumferential stretching ratio; $\mathrm{r}_{\mathrm{f}}$ and $\mathrm{r}_{\mathrm{i}}$ : final and initial radius at horizontal position

Instead of using fluid flow model to create the membrane deformed, different pressure scales were applied. In this perspective, it was assumed that for a small amount of fluid loaded to the cell chamber, the shape of the extending PDMS was similar to the semisphere. In this case, $44 \mu 1$ fluid was applied for $1.3 \mathrm{~cm}$ diameter membrane. This value 
was selected based on the condition of the experiment for the embryonic chick ventricle cell culture that was performed later in chapter 6 . From equation 5 , the maximum deflection (or height) was estimated. This maximum deflection value $(\mathrm{h}=0.33 \mathrm{~mm})$ was used as a reference for the deflection profile of the cell membrane generated by the finite element analysis (FEA).

Finite element software ANSYS 14.5 (Southpointe, PA) was utilized to generate the membrane deflecting profile as a function of applied pressures. The PDMS membrane was modeled using SHELL 281 with 2D, axi-symmetric boundary condition. For this simulation, it was assumed that PDMS membrane was hyper-elastic material; therefore, Mooney-Rivlin strain energy was used to declare the physical properties of PDMS membrane, ${ }^{122}$ and large deflection mode was turned on. The thickness of the membrane was $500 \mu \mathrm{m}$ and a Poisson's ratio was 0.49 . The membrane was assigned with fixed boundary conditions at the edges, and the pressure was applied evenly on the top of the membrane. For each run, a certain pressure value was applied to the membrane until the maximum deflection of the membrane $(\mathrm{h} \sim 0.33 \mathrm{~mm})$ was obtained. Once the displacement profile (Fig. 5.6) was obtained, the radial and circumferential strains of the membrane were determined (Fig. 5.7). The radial strain from the center to the edge was estimated using Eq. 6 and 7, where $\mathrm{R}_{\mathrm{f}}$ and $\mathrm{R}_{\mathrm{i}}$ are the initial and final radius. 

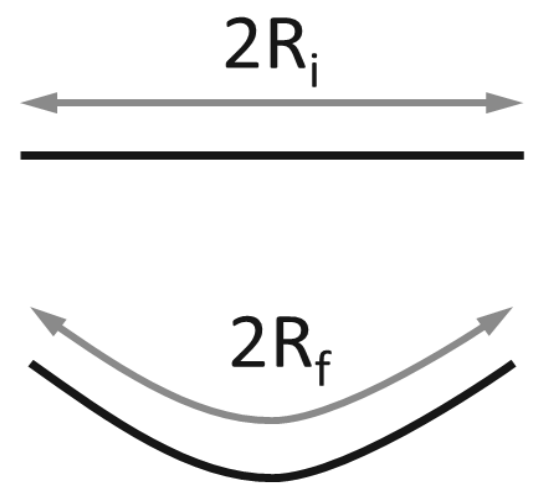

$$
\varepsilon_{r}=\frac{R_{f}-R_{i}}{R_{i}}
$$

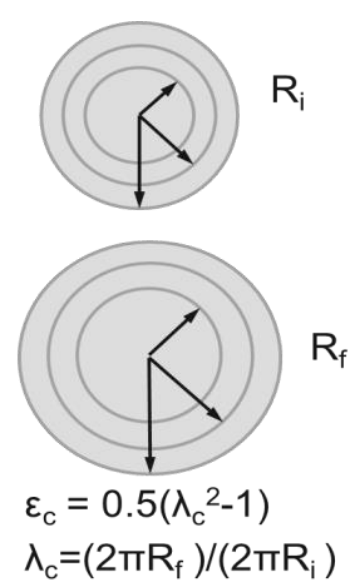

Figure 5.5: Schematic demonstration for radial strain (left) and circumferential strain (right) measurements for a thin PDMS circular membrane.
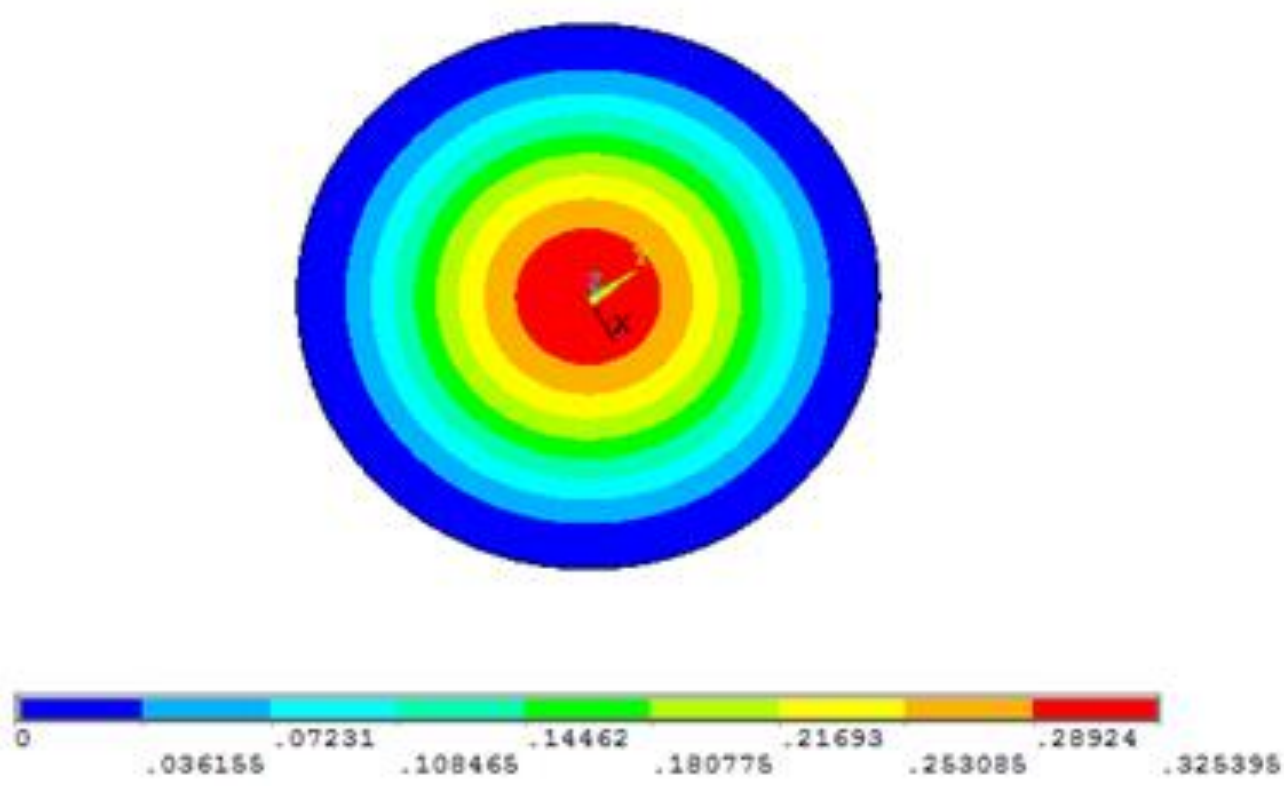

Displacement (mm)

Figure 5.6: The sketch of PDMS membrane deflection $(\mathrm{d}=13.0 \mathrm{~mm}, \mathrm{t}=0.5 \mathrm{~mm})$ using SHELL 281. The maximal displacement of this membrane was $0.33 \mathrm{~mm}$ at the center. 
[A]

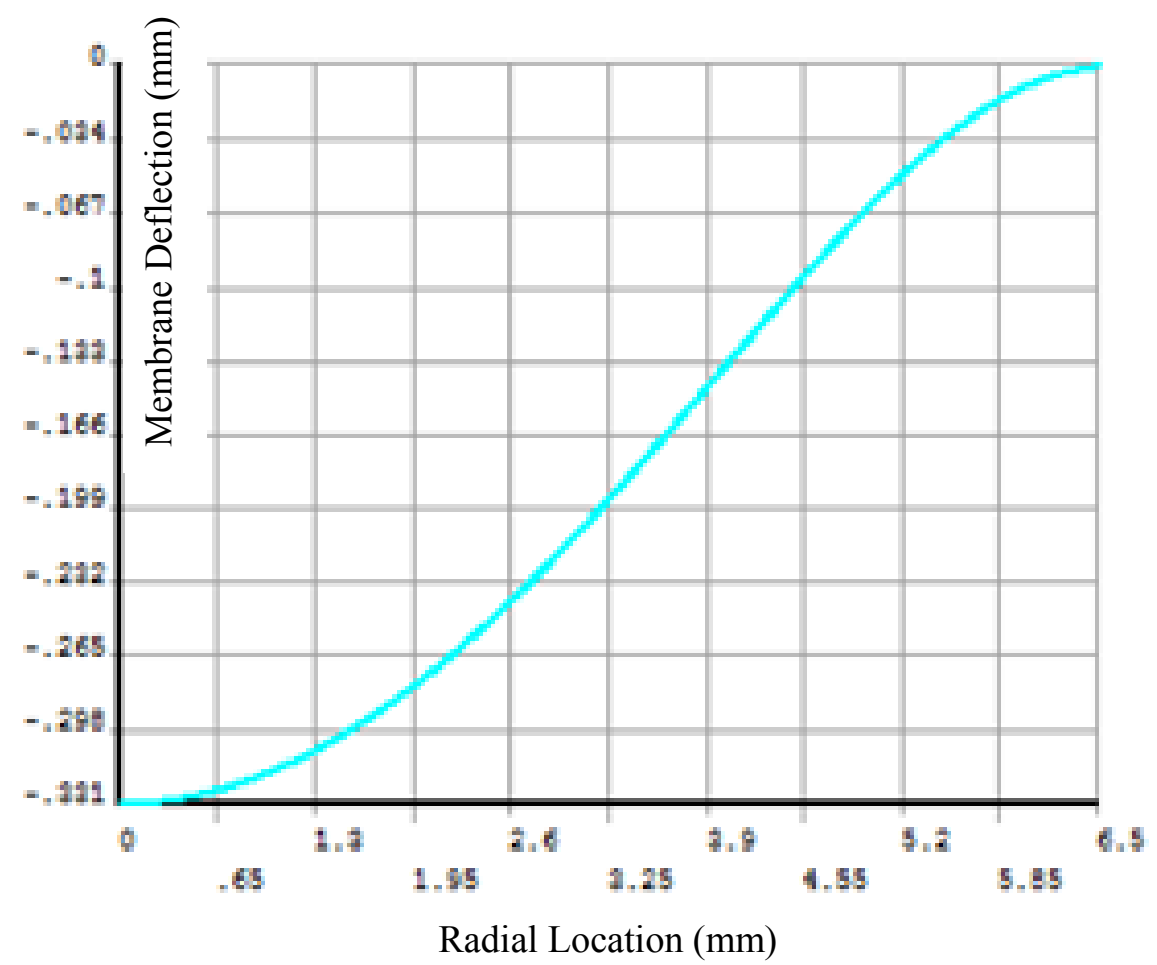

[B]

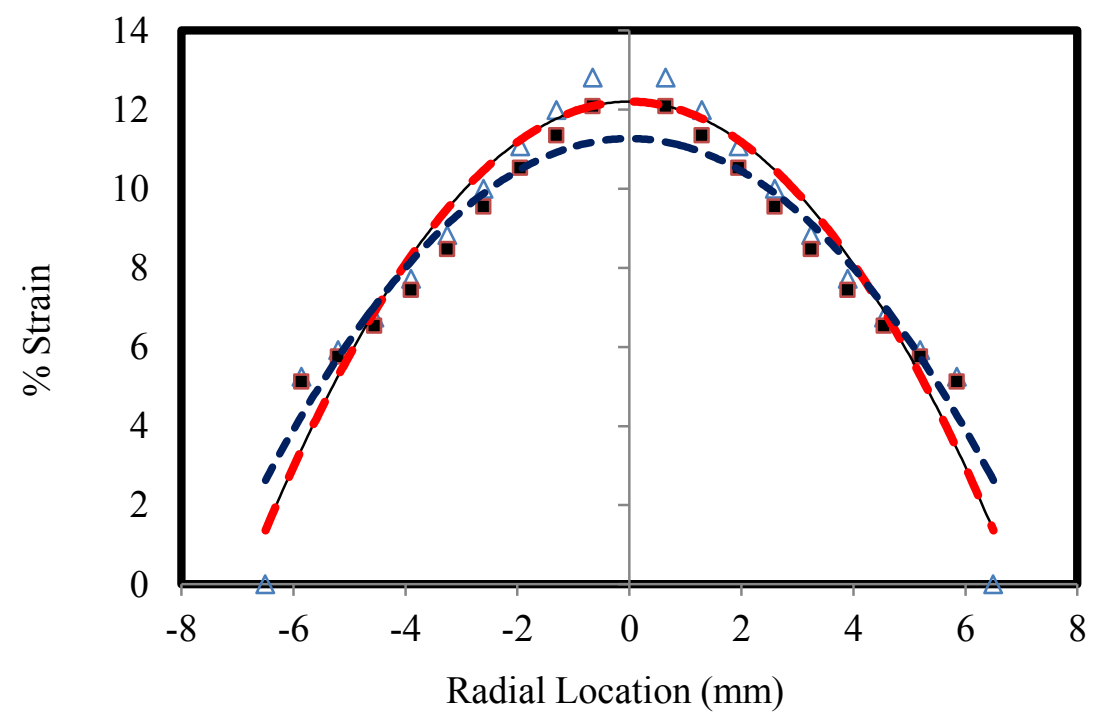

Figure 5.7: Plots of displacement and strain of the circular PDMS membrane. [A] Plot of the PDMS membrane displacement from ANSYS and [B] Estimation of radial strain (A) and circumferential strain ( $\square$ ) on the thin polymeric membrane at the bottom of the cell culture chamber as a function of radial position. 


\subsubsection{Strain measurement via real set-up experiment}

Besides using Finite Element Analysis (FEA) to estimate the membrane deflection and strain ${ }^{122}$, we used high resolution ultrasound Imaging (Vevo-2100, Visual Sonics Inc, Toronto, Canada) to examine the membrane displacement of the real set-up system. A MS-400 probe $(30 \mathrm{MHz})$ was used for recording the cyclic movement of the membrane (Fig.5.8.).

[A]

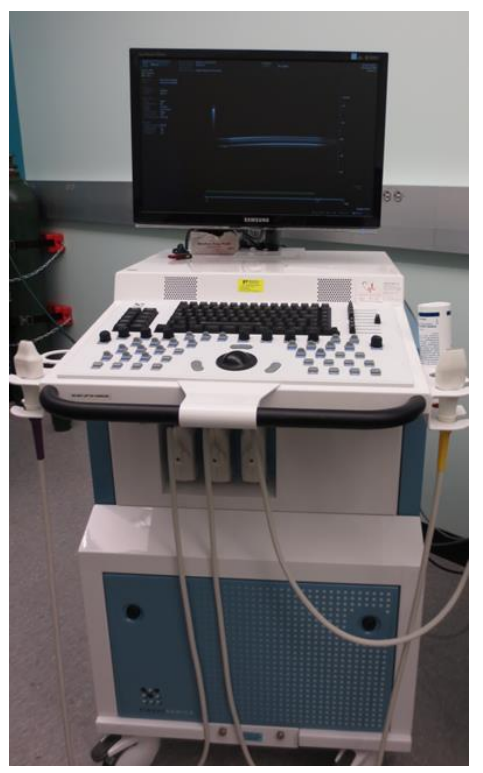

[B]

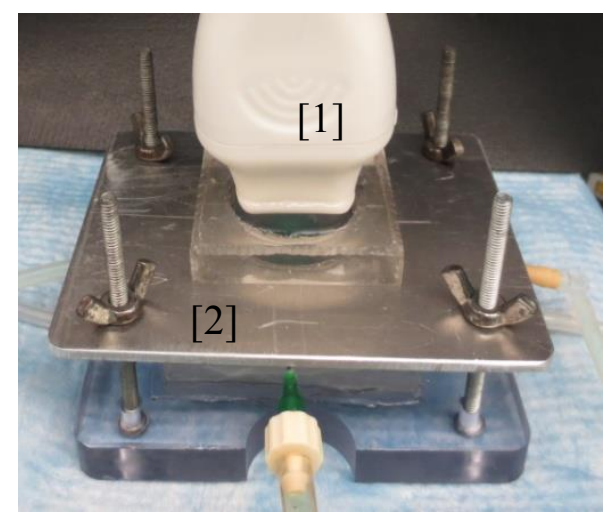

Figure 5.8: Images of experimental set up for taking real-time video of the PDMS membrane deflections using the Ultra Sound system. [A] Image of the Vevo2100 high frequency ultrasound system used for the PDMS strain measurement. [B] Experimental set-up for the strain measurement with [1] probe and [2] CCCM platform where the CCCM culture chamber was installed.

Three different experiments were set up with three different $500 \mu \mathrm{m}$ thick PDMS membranes. The flow rate was set at $44 \mu 1 /$ cycle, the cyclic pressure was from $0 \mathrm{mmHg}$ as 
the base to its peak of $10 \mathrm{mmHg}$, and the frequency was set at $2.0 \mathrm{~Hz}$ with $40 \%$ diastolic phase. To ensure that CCCM system worked correctly and the membrane deflection was in the right range for each membrane, manual ejections of different fluid volumes ( $25 \mu 1$ and $50 \mu 1)$ were performed besides the real set-up experiment (Fig. 5.9.).

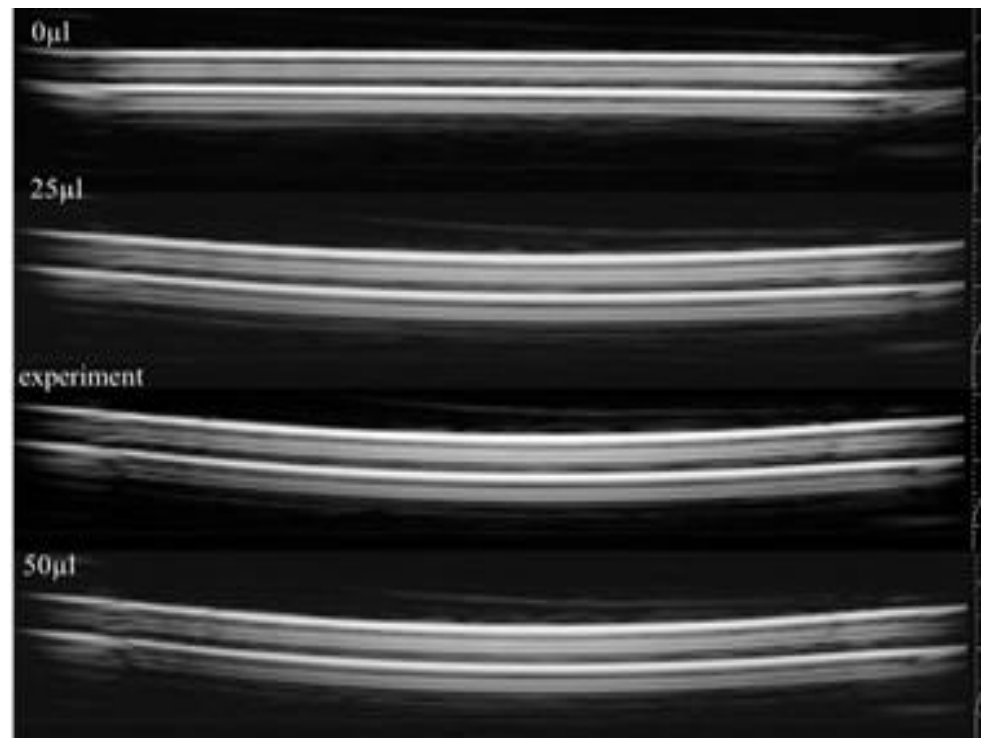

Figure 5.9: Ultrasound images of PDMS membrane deflection at 0,25 , and $50 \mu 1$ fluid loading along with the real set-up experiement. From these images, the level of membrane stretch from the experiment is located between $25 \mu 1$ and $50 \mu 1$ injections.

For each ultrasound movie, the deflecting movement of the PDMS membrane was converted to single moving slides ( 30 slides $/ \mathrm{sec})$. The base slide and the maximum deflecting slides were selected for each measurement (Fig.5.10.). Using an ad hoc program developed in MATLAB (Mathworks, Natick, MA), reference points and displacement points along the membranes from the base line and the maximum deflecting slides were created evenly along the membrane to determine the $R_{i}$ and $R_{f}$. The deflection profile was then created to calculate the strains, and the radial, as well as the 
circumferential strain of these membranes, were therefore calculated using the equations (6) and (7a-b).

\subsubsection{Conclusion}

In addition to the pressure stimulation, stretch levels in cardiac cells play a critical role ensuring cell phenotype and function. In the CCCM system, cardiac cells were attached and grown on a soft matrix which is laid on the thin PDMS membrane. Therefore, the $\%$ strain of the PDMS membrane represents the stretch level of the cells growing on it. With two different methods for strain measurement, the strain profile for the PDMS membranes was found to match the strain experienced by cardiomyocytes within the native ventricle. These results confirm that the PDMS membrane could replicate in-vivo stretch experienced in the heart. 
[A]

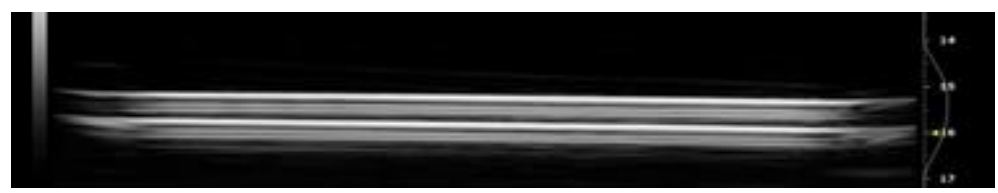

[B]

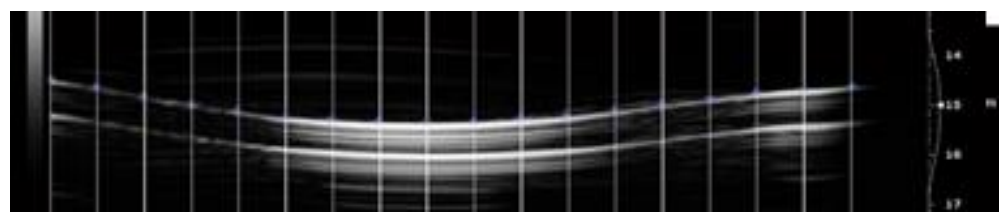

[C]

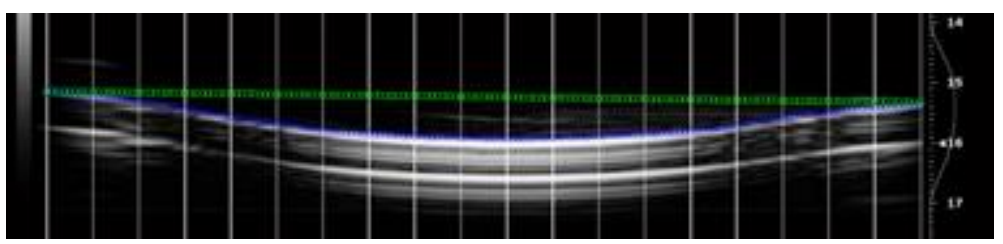

[D]
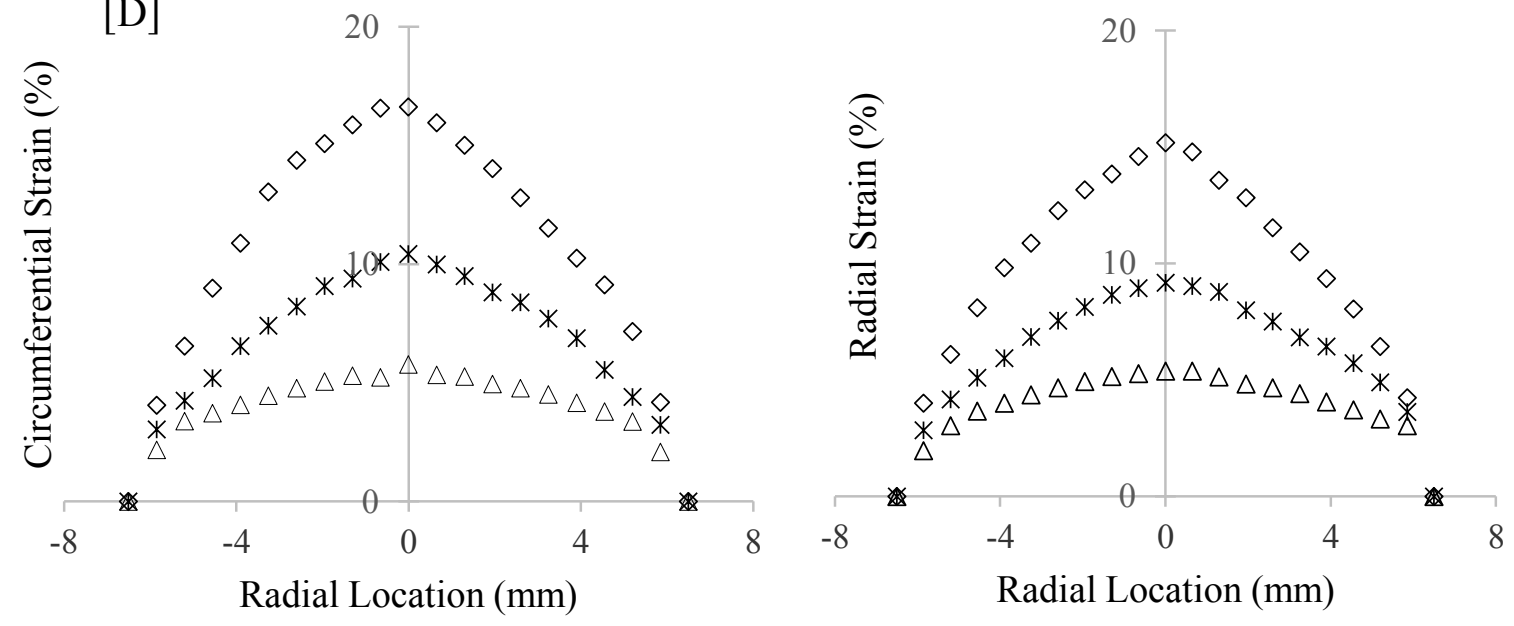

$\diamond 50 \mu 1 \quad *$ experiment $\quad \triangle 25 \mu 1$

$\diamond 50 \mu \mathrm{l} \quad *$ experiment $\quad \triangle 25 \mu \mathrm{l}$

Figure 5.10: Results of the PDMS membrane deflection. [A] Slide of base line where no fluid was loaded to the system. [B] Slide of the maximum PDMS membrane deflection. The vertical lines were created to divide the membrane in even distances between 2 edges. [C] Image of reference points on the base line (green color) and displacement points along with the deflecting curve (blue color). [D] Graphs of circumferential strain (left) and radial strain (right) of the $500 \mu \mathrm{m}$ PDMS membrane. 


\subsection{SHEAR STRESS MODELING}

The dimension of the cell culture well is similar to the dimension of the previous $\mu C C C M$ model. The height of the later model is $0.5 \mathrm{~cm}$ higher than the height of the well in the $\mu \mathrm{CCCM}$ model. Therefore, a design guideline published in the prior work (for the $\mu \mathrm{CCCM}$ system ${ }^{111}$ ) was applied to ensure that the depth of the cell culture wells was $>0.5 \mathrm{~cm}$, resulting in low shear recirculation at the bottom of the wells. This work was performed by Vahidreza Parichehreh, the co-author of the previously published manuscript. ${ }^{111}$ Below is the detail copy of method and results from the previous work for shear stress modeling in the $\mu \mathrm{CCCM}$ system.

\subsubsection{Method}

Finite element software ANSYS Academic Teaching Advanced 12.0, FLOTRON module, was used to determine the wall shears stress as a function of channel height and flow rate. A 2D simulation was applied and environment was verified for steady incompressible flows. For the simulation, it was assumed that no-slip boundary conditions for the channel and groove walls, and that the participating fluid has the same physical properties of water with the density of $1000 \mathrm{~kg} \cdot \mathrm{m}^{-3}$ and the dynamic viscosity of 10 Pa.s. The inlet fluid flow rate was set at $8.6 \mathrm{~mL} / \mathrm{min}$ and zero pressure was applied to the outlet. The fluid domain was meshed using 2D quadrilateral element, FLUID141, to model steady state fluid. A segregated sequential solver algorithm was used; that is, the matrix system derived from the finite element discretization of the governing equation for each degree of freedom was solved separately. The mesh was refined through mesh sensitivity analyses: at each simulation, the elements showing high velocity gradients 
were refined, until reaching convergence of sensitive measures of the predicted quantities.

\subsubsection{Results}

Four wells with different depth $(0.25,2.00,4.00$, and $8.00 \mathrm{~mm})$ were used; and a velocity of $\sim 0.024 \mathrm{~m} / \mathrm{s}$ (corresponding to $8.6 \mathrm{~mL} / \mathrm{min}$ ) at the inlet of the wells was applied in all the experiments. The shear stress $(\tau)$ was determined using the equation ${ }^{131}$

$$
\tau=\mu \frac{d u}{d y}
$$

where $\tau$ is the shear stress $\left(\right.$ dynes $\left./ \mathrm{cm}^{2}\right), \mu$ is viscosity coefficient $(\mathrm{kg} /(\mathrm{m} . \mathrm{s})), \mathrm{u}$ is the fluid velocity $(\mathrm{m} / \mathrm{s})$. The viscosity $(\mu)$ of water at $1 \mathrm{~atm}$ and $20^{\circ} \mathrm{C}$ is $1.0 \times 10-^{3} \mathrm{~kg} /(\mathrm{m} . \mathrm{s}) .^{131}$

\subsubsection{Discussion}

The flow velocity simulation (Fig.5.11.) showed that for the wells with depth of 2.00, 4.00 , and $8.00 \mathrm{~mm}$, with the flow rate of $8.6 \mathrm{~mL} / \mathrm{min}$, the flow velocity was nearly 0.00 $\mathrm{m} / \mathrm{s}$ at the bottom of the well whose height is higher than $0.25 \mathrm{~mm}$. Indeed, the result from ANSYS shows that the shear stress was 0.00 dynes $/ \mathrm{cm}^{2}$ to approximately $200 \mu \mathrm{m}$ above the bottom of the wells with the height higher than $8.0 \mathrm{~mm}$. The second generation CCCM system has the same diameter of cell chamber and same dimensions of fluid inlet and outlet; but the height of the chamber is about $10.00 \mathrm{~mm}$. With this height, definitely, fluid flow will not create a significant shear stress, affirming that cell cultured in the second generation CCCM won't expose to the shear stress. In addition, this characterization confirmed that this system can be able to manipulate the shear stress based on the height of the cell chamber and the velocity of the fluid. 
(a) Well Depth: $0.25 \mathrm{~mm}$

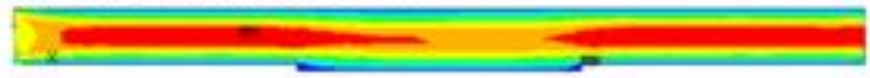

(b) Well Depth: $2 \mathrm{~mm}$

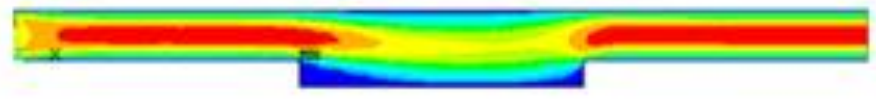

(c) Well Depth: $4 \mathrm{~mm}$

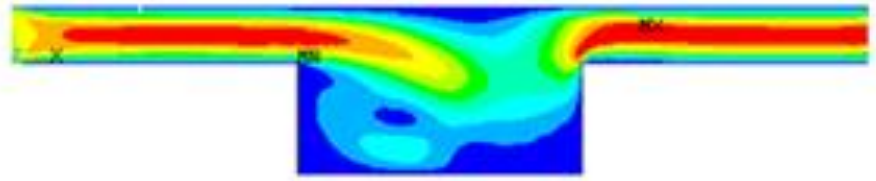

(d) Well Depth: $8 \mathrm{~mm}$

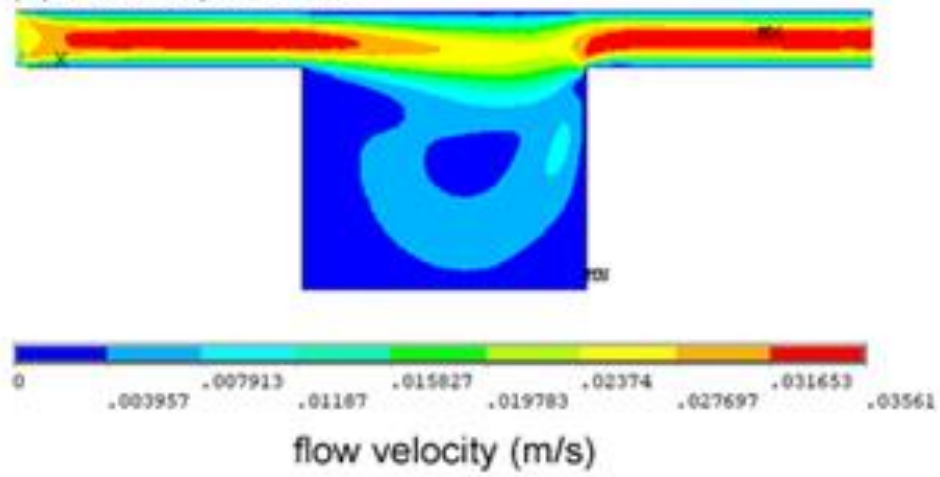

Figure 5.11: Flow velocity simulation for the cell culture chamber of the $\mu$ CCCM. Note that the flow velocity are almost $0.00 \mathrm{~m} / \mathrm{s}$ at the bottom of the wells except the $0.25 \mathrm{~mm}$ deep well, meaning fluid movement does not touch the bottom of these wells; therefore, shear stress is negligible at the bottom region where cells are cultured.

\subsection{CONCLUSION}

Overall, the characterization of mechanical properties of thin PDMS membrane and pressure, strain, and shear stress affirmed strongly that the CCCM can be used to replicate the in vivo heart function. This replication can be accomplished through a combination of different variables such as membrane thickness, the height of the cell 
chamber and adjustment of tunable components, including the tunable valve, the peristaltic pump, and the pressure generator. 


\section{CHAPTER 6}

\section{USING THE CCCM SYSTEM FOR CARDIOMYOCYTES STUDIES}

\subsection{BUILDING THE EXTRACELLULAR MATRIX FOR CARDIAC CELL \\ CULTURE}

\subsubsection{Introduction}

The development of scaffolds that mimic the in-vivo extra cellular matrix (ECM) is crucial for the engineering tissue remodeling/regeneration. The ECM provides a microenvironment for cells to grow, proliferate, and migrate. The biochemical, structural, and mechanical properties of scaffolds play a significant role in regulating cell adhesion to ECM and to neighboring cells, which in turn affect cell morphogenesis and differentiation. $^{132-137}$ It is true especially in the case of in-vitro cardiomyocyte studies, the beating rate, force of contraction, and cytoskeleton structure of cardiac myocytes which depend greatly on the ECM. ${ }^{132,133,138,139}$ Therefore, cardiac cells need to be seeded and grow on a soft cell culture matrix, similar to the in-vivo heart tissue. In this study, collagen matrix was used. Based on the paper published, ${ }^{16,140}$ the cell culture matrix used for cardiac tissue was determined via the morphology and spreading of cells similar to the in-vivo physiology. Therefore, the concentration of the collagen used for constructing matrix was $0.667 \mathrm{~g} / \mathrm{ml}$ collagen. ${ }^{16,140}$ 
In addition, to ensure that the collagen ECM was not rapidly digested by the cardiomyocytes during the experiment, crosslinking with glutaraldehyde was applied to the matrix. Glutaraldehyde is a cross-linking agent that is commonly used for collagenbased biomaterial. ${ }^{141,142}$ Finally, for the cell to attach and grow on the cross-linked collagen ECM, a gelatin solution containing fibronectin was also added to the matrix prior to the cells seeded.

\subsubsection{Fabrication of the ECM}

First, the sterilized PDMS cell culture chamber was treated with oxygen plasma to create a hydrophilic surface (March RIE, $200 \mathrm{mmTorr}, 200 \mathrm{mmHg}$, 10\% oxygen, 30 seconds). Then, a $300 \mu \mathrm{L}$ of the collagen type I solution $(0.67 \mathrm{mg} / \mathrm{mL})$ was promptly added to the cell culture chamber and allowed to be cured at $37^{\circ} \mathrm{C}$ for $3-4 \mathrm{~h}$. Second, a $400 \mu \mathrm{L}$ of $0.5 \%(\mathrm{w} / \mathrm{v})$ glutaraldehyde solution was gently added on the surface of the cured collagen matrix and maintained for $1 \mathrm{~h}$ at room temperature (RT). After $1 \mathrm{~h}$, the matrix was washed with deionized (DI) water three times, followed by a $4 \mathrm{~N}$ sodium hydroxide solution twice (30 min/each). Finally, DI water was used again for the final wash (three times). The cell chamber was filled with DI water and stored at $4{ }^{\circ} \mathrm{C}$ for later use. $48 \mathrm{~h}$ before cells were seeded, a $400 \mu \mathrm{L}$ solution composed of $10 \%$ gelatin attachment factor (1X, Gibco, S- 006-100) and $50 \mu \mathrm{g} / \mathrm{mL}$ of fibronectin (BD Biosciences, 356008) was added to the chamber. A one-time washing with $1 \mathrm{X}$ PBS or fresh culture medium was performed before the cells were seeded. 


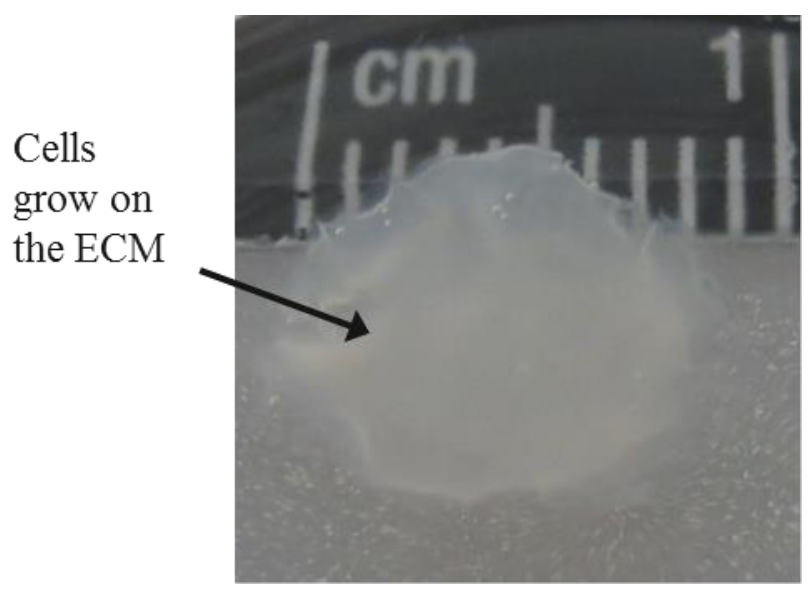

Figure 6.1: Image of the collagen matrix with cardiac cells after 4 day cultured in the CCCM system.

\subsection{CULTURING CARDIOMYOCYTES IN THE CCCM SYSTEM}

\subsubsection{Primary embryonic chick ventricular cardiomyocytes}

Embryonic chick cardiac cells are commonly used to study the complex processes leading to normal or abnormal heart development. Chick embryonic heart development is quite similar to that of the human heart. Fertile chick eggs are relatively easy to obtain and less expensive than rodent models. Finally, it is possible to observe the embryonic heart at the earliest stage of its embryogenesis and to isolate embryonic cardiomyocytes for in-vitro culture. ${ }^{15,79,143}$

During the embryonic period, cardiomyocytes have a high proliferation rate and differentiation. ${ }^{16}$ The synthesis of DNA from the embryonic cardiomyocytes reaches its peak during the cardiac morphogenesis. During this developmental window, mechanical hemodynamic loading and unloading play a crucial role in regulating the cardiomyocyte 
morphogenesis, architecture, and contractile functions and stimulating different molecular signal pathways. On the other hand, in response to these mechanical stimulations, embryonic cardiomyocytes themselves regulate their proliferation and differentiation rate. Therefore, any changes in mechanical hemodynamic loading and unloading system affect the development of cardiomyocyte tissues and their contractive functions greatly.

Chick embryonic heart development can be divided into 46 stages, called Hamburger Hamilton (HH) stages. ${ }^{79,} 144$ These stages are used rather than only using days of incubation due to variations in maturational rate that occur in response to changes in ambient environmental factors. The first myocardial differentiations are detected on stage $\mathrm{HH} 7$ when the formation of the endocardial heart tube begins. On $\mathrm{HH} 8$, the process of heart tube fusion starts, and is completed on HH 11. Heart beat begins on this $11^{\text {th }} \mathrm{HH}$ stage. During stages between the 13 to the 18 , the heart tube transforms to the $\mathrm{C}$ shape loop then to the S-shape loop to form atria and ventricles. HH 18-22, the primary ventricular channels can be observed. HH 21-34, valve development, vasculature, atrial and ventricular septations are gradually formed, along with other cardiac components, such as the cardiac conduction system, atrial-ventricular (AV) nodes or cardiac neural crest. Ventricular septation is completed around HH 34. HH 36 (or day 10 gestation), the Purkinje fiber network begins to form and the atrial-ventricle valve is completely formed to separate the atria from the ventricle. HH 40-41 is the hatching stage. Besides the HH stage, the stages of embryonic heart development can also be named based the incubation day. The chick hatches on approximately the 21 st day of incubation. Primary 
cardiac morphogenesis occurs between day 2 to day 10 of a 21 -day gestation. Day 10 is corresponding with HH36, where the ventricles are completely separated. Therefore, we selected $10^{\text {th }}$ day embryonic chick ventricles for cell isolation in our CCCM experiments.

Embryonic cardiac development depends on the integration of the environmental conditions, biomechanical forces, molecular programming, and adaptive remodeling. Cyclic mechanical stimulation inside the heart induces myocardial hyperplasia. In addition, in the early period of cardiac morphology, the function of chick embryonic cells, including the adaption of the contractile function, is greatly affected by mechanical loading conditions. Mechanical stimulation thus affects the formation of the shape, as well as the growth of heart chambers. Indeed, applying mechanical stimulation during different stages of the embryonic period will greatly affect the function of the heart tissue, which includes cell proliferation, contraction, frequency, and the duration of contraction. Heart cells start beating synchronically and strongly, similar to the in-vivo nature, reached the peak in-vitro on culture day $12 .{ }^{16}$ Therefore, the heart embryonic tissue increased active contractile force from culture day 7 to day 12. Also, culture day 12 has the highest proliferation. However, the contractile response to the $\beta$-adrenergic stimulation was less than culture day 9. Under these studies, 3 different developing stages were standardized for mechanical stimulation: from culture day 4 to 6 (before the cells increase the contraction force), from culture day 9-11 (before the proliferation goes down), and at culture day 13-15 (the transition between embryonic and fetal stages). 


\subsubsection{Embryonic chick cardiomyocyte isolation and culturing}

\subsubsection{Embryonic Chick Cardiomyocyte Isolation and seeding}

Fertile White Leghorn chicken eggs (Utah) were incubated in a forced-draft, constanthumidity incubator until day 10 of the gestation $(\mathrm{HH}$ : stage 36). Ventricles were collected and enzymatically digested using $2 \mathrm{mg} / \mathrm{mL}$ of collagenase type II, followed by $0.05 \%$ trypsin-EDTA solution (Invitrogen, Carlsbad, CA). The cell solution was then filtered and isolated cells were collected. An $1 \mathrm{~h}$ pre-plate for the isolated cells at $37^{\circ} \mathrm{C}$ was performed in $10 \mathrm{~mL}$ of cell culture medium (DMEM high glucose, 10\% Fetal Bovine Serum, $1 \%$ embryonic chick extract, and 1\% antibiotic), followed by a 5 min centrifuge at $1000 \mathrm{rpm}$. Cell pellet was then re-suspended in a new $10 \mathrm{~mL}$ of cell culture medium and were cultured on a gyratory shaker $(60-70$ rotations/min) for $18 \mathrm{~h}$ to re-aggregate viable cardiomyocyte clusters. The following day, the whole cells, clusters, and medium were collected and spun at 1000rpm for $5 \mathrm{~min}$. Old medium was discarded and $1 \mathrm{ml}$ of warmed fresh medium was added. Cell clusters were counted and cell viability was checked with Trypan blue staining. For each cell culture chamber prepared from the session 6.1 .2 , approximate $500 \mu 1$ of $3.0-3.5 \times 10^{4}$ cell clusters were seeded and cultured in a static condition at $37{ }^{\circ} \mathrm{C}, 5 \% \mathrm{CO}_{2}$ for $3 \mathrm{~h}$ before filling the reservoir with warmed fresh culture medium. A static culture condition at $37^{\circ} \mathrm{C}$ and $5 \% \mathrm{CO}_{2}$ was maintained for $24 \mathrm{~h}$ to obtain $100 \%$ confluence. On the following day, the sample was subject to mechanical loading within the CCCM, or maintained under static conditions as a control. 


\subsubsection{Culturing cardiac cells under the influence of mechanical stimulation within the CCCM}

Day 10 embryonic chick cardiomyocytes experience in-vivo peak pressures of $\sim 10-15$ mmHg and radial and circumferential strains of $\sim 10-20 \% .{ }^{130}$ Since the cardiac cell tissue is thinner and weaker than the in-vivo cardiac tissue, a peak systolic pressure of 10 $-12 \mathrm{mmHg}$ and a strain of $8-15 \%$ were chosen for the whole experiment. To culture cardiomyocytes under these conditions, a $500 \mu \mathrm{m}$ thick membrane was selected and a flow rate of $\sim 2.6 \mathrm{ml} / \mathrm{min}$ was used to attain a filling volume of $44 \mu \mathrm{l}$ per cycle.

Following $24 \mathrm{~h}$ of culturing under static conditions to achieve $100 \%$ confluence, the cell culture chamber with cells was transferred to the CCCM system and the system was assembled as described in the section 4.3. The whole set-up CCCM system was then moved into the cell culture incubator and was ready for mechanical stimulation for 4 days at $37{ }^{\circ} \mathrm{C}$ and $5 \% \mathrm{CO}_{2}$. The static pressure inside the cell culture chamber was reported before the peristaltic pump was turned on. The flow rate was set at $\sim 2.6 \mathrm{ml} / \mathrm{min}(\sim 44$ $\mu 1 /$ cycle). The downstream one-way valve and the pressure from the generator were adjusted to obtain cyclic pressures of $0-10 \mathrm{mmHg}$ at a frequency of $2.0 \mathrm{~Hz}$. Pressure waveforms were checked in a period during 4 day stimulation. For comparison, the control samples were cultured under a static condition in the same culture incubator with the same amount of fresh culture medium. After 4 days, the cell culture chamber was removed from the CCCM platform and then maintained under a static condition for $\sim 3 \mathrm{~h}$ prior to analysis. 


\subsubsection{Results}

During the CCCM stimulation, the instantaneous pressure generated inside the cell chamber was obtained using a high fidelity pressure transducer and LabView software. Based on the percentage of systolic versus diastolic timing set on the pressure generator, along with the pressure waveform and fluid volume loaded to the chamber, the pressurevolume loop for a single working cycle of the CCCM was obtained (Fig. 6.2.).

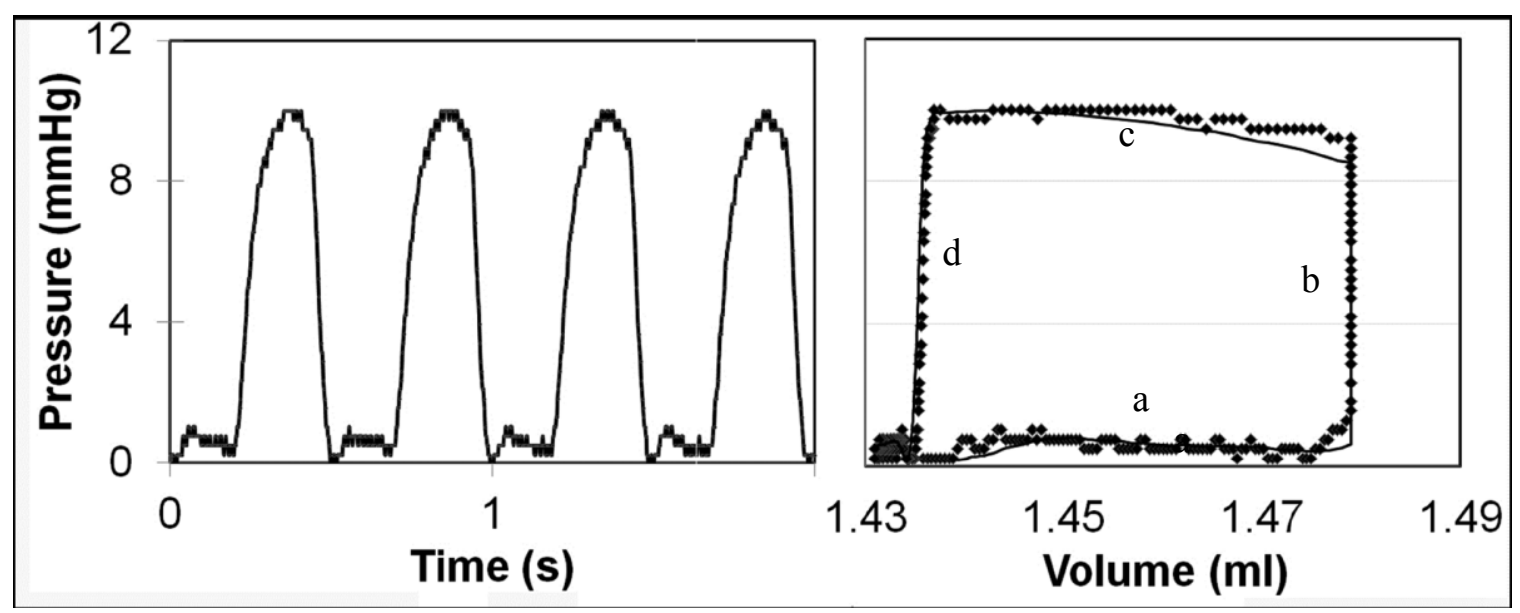

Figure 6.2: Plot of pressure vs time and pressure-volume loop obtained within the CCCM system. The changes in pressure within the CCCM cell culture chamber and pressure-volume loop correlating changes in pressure with changes in volume within the CCCM chamber was determined. The parameters of the pressure and fluid volume were set up based on the parameters from the left ventricle of the embryonic chick heart. Similar to the pressure-volume loop seen in the left ventricle of the heart, this pressurevolume loop represents the (a) filling phase, where the pressure was low but the volume increased, (b) the isovolumic contracting phase, where the pressure increases significantly but the fluid volume was maintained the same during this phase, (c) the 
ejection phase, where the fluid was ejected out of the chamber, and (d) the isovolumic relaxing phase where the pressure drop significantly with no change in the fluid volume.

After 4 days in the mechanical stimulations, the tissues were removed from the system, followed by tissue analysis. Observed under the microscope, the stimulated construct has more cardiac islands on the surface and looked more crowded and rougher, while the control has a flatter surface with larger sized cells (Fig.6.3.).
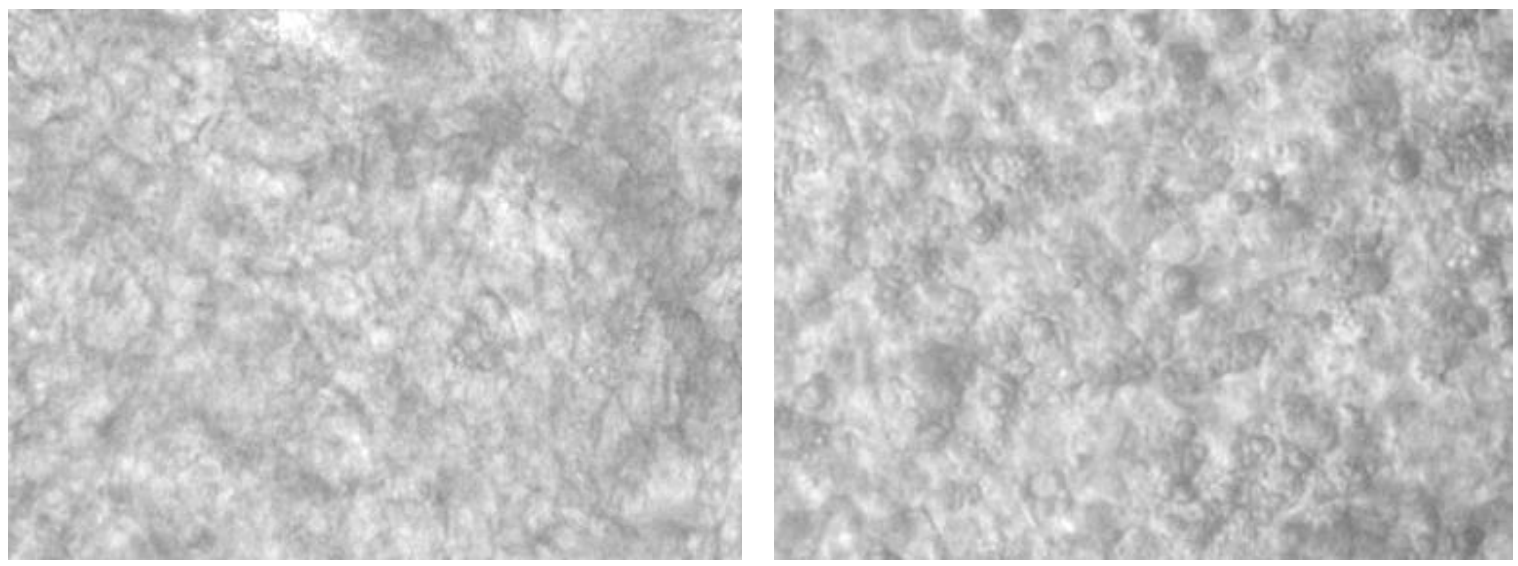

Figure 6.3: Phase contrast images of in-vitro embryonic chick cardiac cells cultured under the static and CCCM stimulated conditions. Left: embryonic chick cardiac cells cultured under the static condition as a control. Right: embryonic chick cardiac cells cultured under mechanical stimulations generated within the CCCM system. Compared to the control cardiac tissue, the conditioned cells appeared to have higher cellular density. 


\subsubsection{Conclusion}

The CCCM system is able to mimic the mechanical conditions which occur in the left ventricle. Primary chick embryonic left ventricle cardiomyocytes are able to grow well under the influence of cyclic mechanical stimulation generated within the CCCM system. Further analysis between the stimulated and the static cultured groups will be described below, included tissue alignment, beating rate, maturation, proliferation, and gene expression.

\subsection{CARDIAC TISSUE ALIGNMENT AND PROLIFERATION}

\subsubsection{Introduction}

Cardiomyocytes continuously experience the stress and the change in length during the heart cycle, due to the blood loading and unloading. Cardiac tissues are elongated during diastolic phase and shortened during the systolic phase of a heart. These hemodynamic stress and tissue strain affects cell proliferation and cytoskeletal remodeling. ${ }^{84}$ Indeed, in response to the strain and stress, cardiomyocytes have the ability to remodel cell shape by adding the sarcomere in series or filaments in parallel respectively to maintain cardiac

function. ${ }^{145}$ In addition, cardiomyocytes during embryogenesis have a high proliferation. Staining nucleus with Bromodeoxyuridine (BrdU) along with DAPPI is a common method to check the cell proliferation.

$$
\text { Proliferation }=\frac{\text { total BrdU nucleus }}{\text { total DAPPI nucleus }} 100 \%
$$




\subsubsection{Methods and materials}

Immunofluorescence staining was used to evaluate the cell alignment and proliferation. To examine the cell alignment, the constructs were stained with F-actin and $\alpha$-actinin. Before staining, the samples were fixed with 4\% paraformaldehyde (PFA) at room temperature (RT) for 20 min, followed by a washing with $1 \mathrm{X}$ phosphate buffered saline (PBS) solution. A $0.1 \%$ Triton solution was then added to the constructs for $30 \mathrm{~min}$, RT. A solution of $0.5 \% \mathrm{BSA}$ in $1 \mathrm{X}$ PBS was used as a blocking solution. For F-actin staining, the samples were incubated with TRITC-conjugated Phaloidin (1:100; Millipore, Billerica, MA) in the blocking solution for $1 \mathrm{~h}$ at RT. For $\alpha$-actinin, primary antibody cardiac $\alpha$-actinin EA53 IgG1(1:400, Abcam, Ab7817) and second antibody antimouse (1:600, 488 green, Invitrogen, A21121) in 1\% BSA/1X PBS solution were applied individually to the tissue for $1 \mathrm{~h}, \mathrm{RT}$, alternated with a washing buffer $(0.05 \%$ Tween-20/1X PBS) $1 \mathrm{~h}$ in between. DAPPI (1:100, Millipore, 90229) was also applied to stain the nucleus at the end. Finally, the tissue constructs were mounted with anti-fade solution (Invitrogen, San Jose, CA), and imaged using a Nikon A1 confocal microscopy system (Nikon Instruments, 284 Melville, NY).

To examine cell proliferation, Bromodeoxyuridine (BrdU) incorporation during DNA synthesis was utilized. Basically, $48 \mathrm{~h}$ prior to the tissues being fixed with PFA, $20 \mu \mathrm{M}$ of BrdU (Sigma) was added to the cell culture medium both for the control and the stimulated tissues. After being removed from the present culture environment, the tissues were fixed with $4 \%$ PFA solution for 20 min at RT and permeablized with $0.1 \%$ Triton solution for 30 min at RT. A one hour washing with 1X PBS solution was performed 
before the $\mathrm{HCl}$ solution $(1 \mathrm{~N})$ was added directly to the tissue on ice for $10 \mathrm{~min}$. A $1 \mathrm{~N}$ $\mathrm{HCl}$ solution was then replaced by $2 \mathrm{~N} \mathrm{HCl}$ solution. After sitting at $\mathrm{RT}$ for $10 \mathrm{~min}$, the tissues in the $2 \mathrm{~N} \mathrm{HCl}$ solution were incubated at $37^{\circ} \mathrm{C}$ for an additional $20 \mathrm{~min}$. A one hour washing with 1X TBE buffer (10X TBE, Mediatech Inc., Manassas, VA, 46-011$\mathrm{CM})$ at RT was performed before the tissues were blocked with a blocking solution (1\% $\mathrm{BSA}+0.1 \%$ glycine in $1 \mathrm{X}$ PBS) for $1 \mathrm{~h}$ at $\mathrm{RT}$. The tissues were then incubated with the primary antibody Anti-BrdU (1:100, Abcam, ab1893) in the blocking solution for $24 \mathrm{~h}$ at RT. After $1 \mathrm{~h}$ washing with the washing buffer, the tissue was incubated with secondary antibodies, anti-sheep (1:400, red, Abcam, Ab6743) in the blocking buffer for $1 \mathrm{~h}$ at RT, followed by another $1 \mathrm{~h}$ washing. DAPI (1:100, Millipore, 90229) was then added to stain the nucleus at the end. Finally, the tissue constructs were mounted with anti-fade solution (Invitrogen, San Jose, CA) and imaged using a Nikon A1 confocal microscopy system (Nikon Instruments, 284 Melville, NY).

\subsubsection{Results}

Specimens were stained with F-actin and cardiac alpha-actinin antibodies, and then examined under the confocal microscopy with $60 \mathrm{X}$ magnification. Compared to the control group, embryonic chick heart cells in the CCCM tissue samples were more aligned and oriented (Fig.6.4.). The parallel orientation showed up obviously in the CCCM construct stained with F-actin. The cell arrangement of the control sample appeared to be random. 


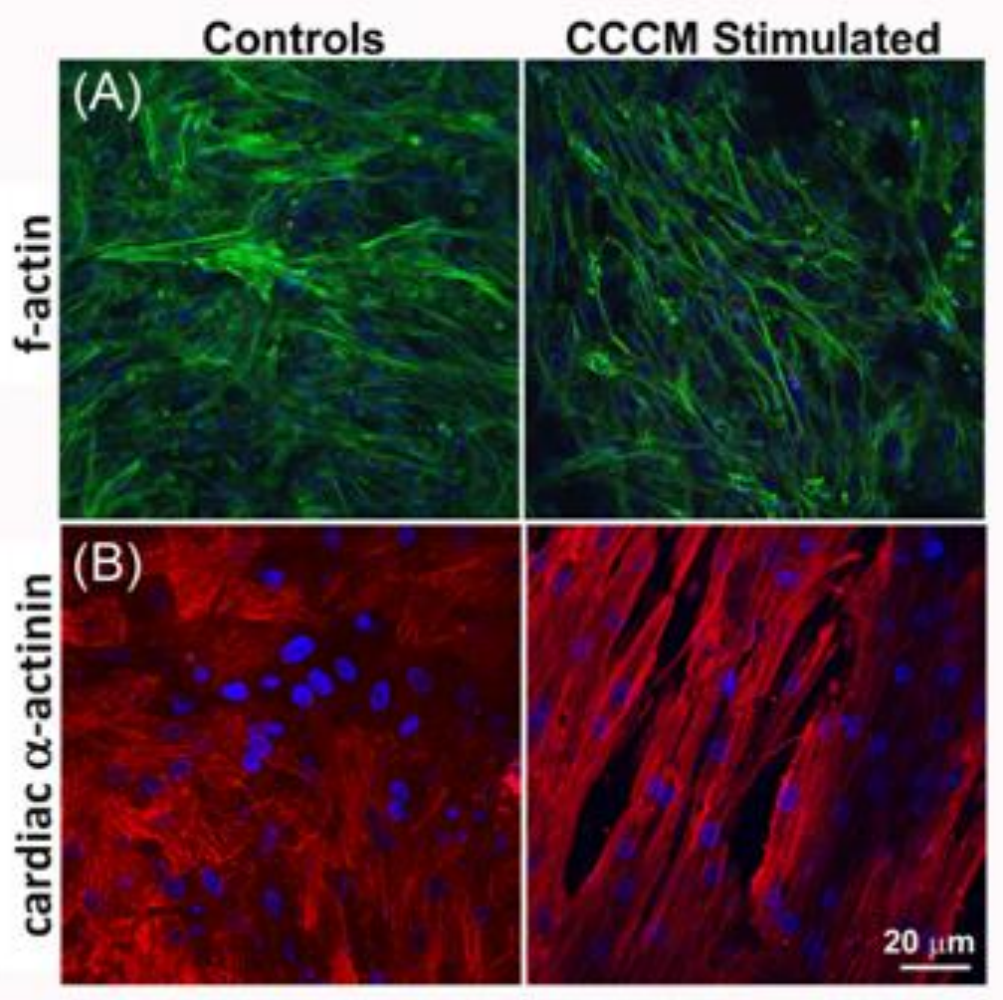

Figure.6.4: Immunofluorescence images of cardiomyocyte tissue cultured in the CCCM system and in the static condition. (A) Images of the cell skeleton structure with f-actin (green). The stimulated tissue cultured in the CCCM system gave more parallel arrangement compared to the random arrangement of the control tissues cultured under the static condition (B) Images of cell alignment with $\alpha$-sarcomeric actinin (red). The picture of the CCCM construct shows in-vivo like matured tissues with stripped and closer connection between cell-to-cell connections. The blue color is the nucleus stained with DAPPI.

To determine the cell proliferation, BrdU, the marker stained for nuclei that have synthesized new DNA during the BrdU treatment period, was used. Different locations 
of the constructs were observed under $60 \mathrm{X}$ magnification of the confocal microscope (Fig.6.5.). The dividing cells had nuclei that showed both blue and red colors overlapping each other, while the undivided cells showed only blue nuclei. Red nuclei without blue were the dead cells. The cell proliferation rate was determined by the ratio between the dividing cells to the total of living cells. Six different experimental sets were done for data statistical analysis.

[A]
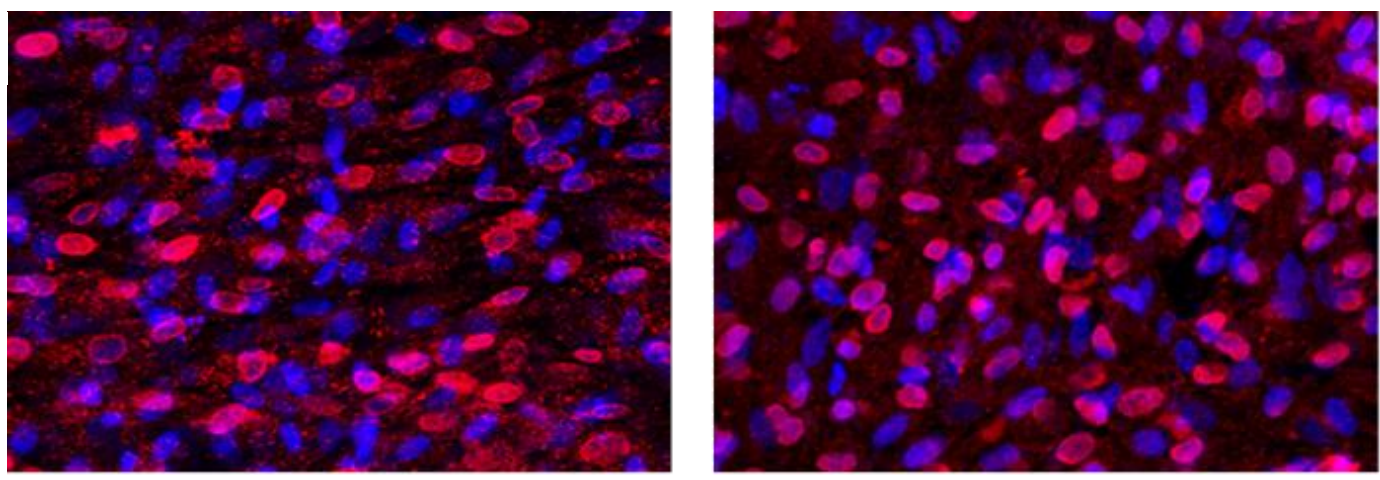

Control

Stimulated

[B]

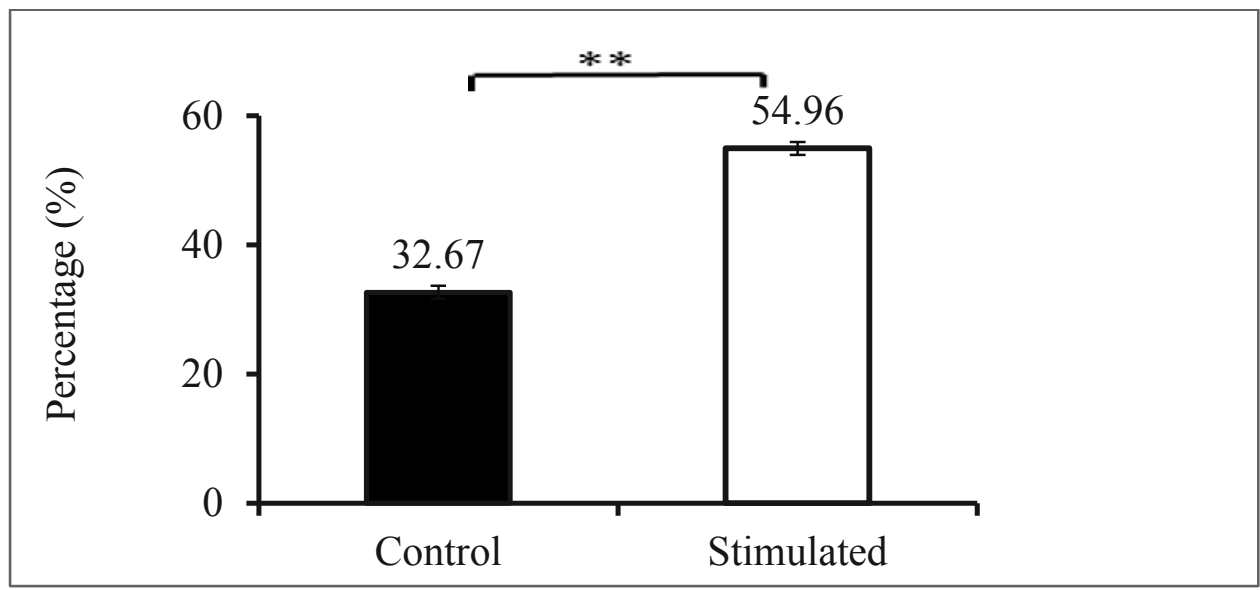

Figure 6.5: Results from immunofluorescence BrdU staining for cell proliferation. [A] Immunofluorescence images of BrdU staining. Red: the nucleus stained with the BrdU antibody; blue: nucleus stained with DAPPI. The dividing cells had both blue and red nuclei; the undivided cells showed only blue nuclei; dead cells showed only red nuclei without blue. [B] Graph of cell proliferation in controls and CCCM stimulated samples. 
The sample size was $\mathrm{N}=6$ with two tailed significance was set at $* * \mathrm{p} \leq 0.05$, meaning a strong chance of having statistical significance between these 2 groups.

\subsubsection{Discussions and conclusions}

The alignment of cardiomyocytes and the cell proliferation were evaluated via immunefluorescence microscopy. Cardiomyocytes were subjected to mechanical stimulation. Cell alignment expresses more in mature cells. Therefore, cardiomyocyte maturation was observed under the immunofluorescence images via Phaloidin and sarcomeric $\alpha$-actinin. The result shows that the contraction-stretching of the cells promotes the increasing of Factin and helps to align the cells. Cell alignments demonstrated that mechanical loading spontaneously rearranged the cytoskeletal orientation of the cells. In addition, from BrdU staining, a higher proliferation rate was observed on the CCCM tissues rather than on the control ones. This result suggests that applying mechanical stimulations on the embryonic cardiac tissue is necessary to promote the cell alignment and proliferation rate of the cardiomyocytes.

\subsection{CARDIAC TISSUE MATURATION}

\subsubsection{Beat rate}

Beat rates $(\mathrm{BR})$ of the conditioned and control constructs were obtained after being removed from the $\mathrm{CCCM}$ for 3 hours. Two different time frames of the $\mathrm{CCCM}$ stimulations (4 and 7 days in the CCCM system) were performed. At least 10 different experiments on different dates were performed. The BR of the stimulated samples was

almost 1.5 times higher than the BR of control samples (Fig.6.6). In addition, the BR of 
the stimulated samples cultured in the CCCM system for 7 days yielded a little higher than the BR of samples with 4 days stimulation, whereas the controls of both groups are the same. This indicates that mechanical stimulations are important to maintain and enhance the BR of the cardiac tissue.

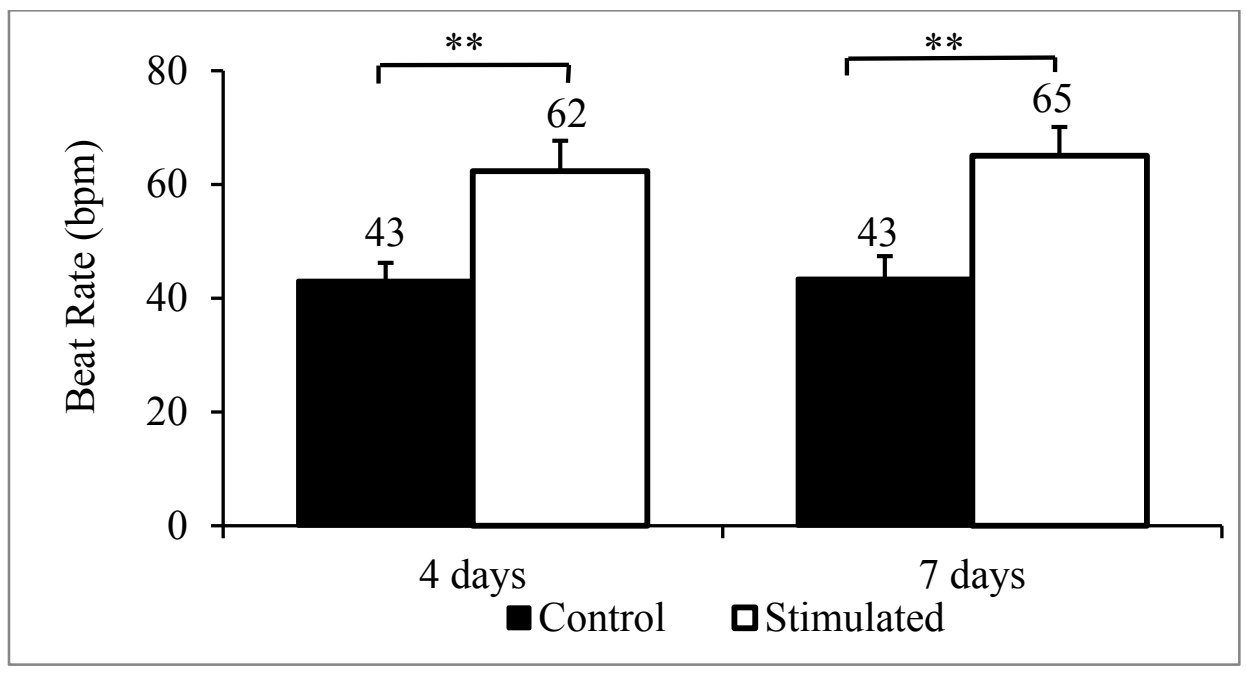

Figure 6.6: Graph of the beat rate (beats per min) between the control and the stimulated groups. 2 different periods of mechanical stimulations (4 days and 7 days) were performed along with their controls cultured under a static condition. Number of samples was $\mathrm{N}=10$. Two tailed significance was statically set at $* * \mathrm{p} \leq 0.005$.

\subsubsection{Electrical Pacing}

Electrical pacing of stimulated constructs and unstimulated controls was accomplished to identify changes in electrical properties in response to mechanical conditioning in the CCCM. Platinum wire electrodes $(\mathrm{d}=0.5 \mathrm{~mm}$, Alfa Aesar, Ward Hill, MA, 43288) were embedded vertically into the tissue-collagen gel matrix and connected to an electrical pacing system (Isolated Programmable Stimulator, Colbourn Instruments, 02142-1) (Fig. 6.7). 


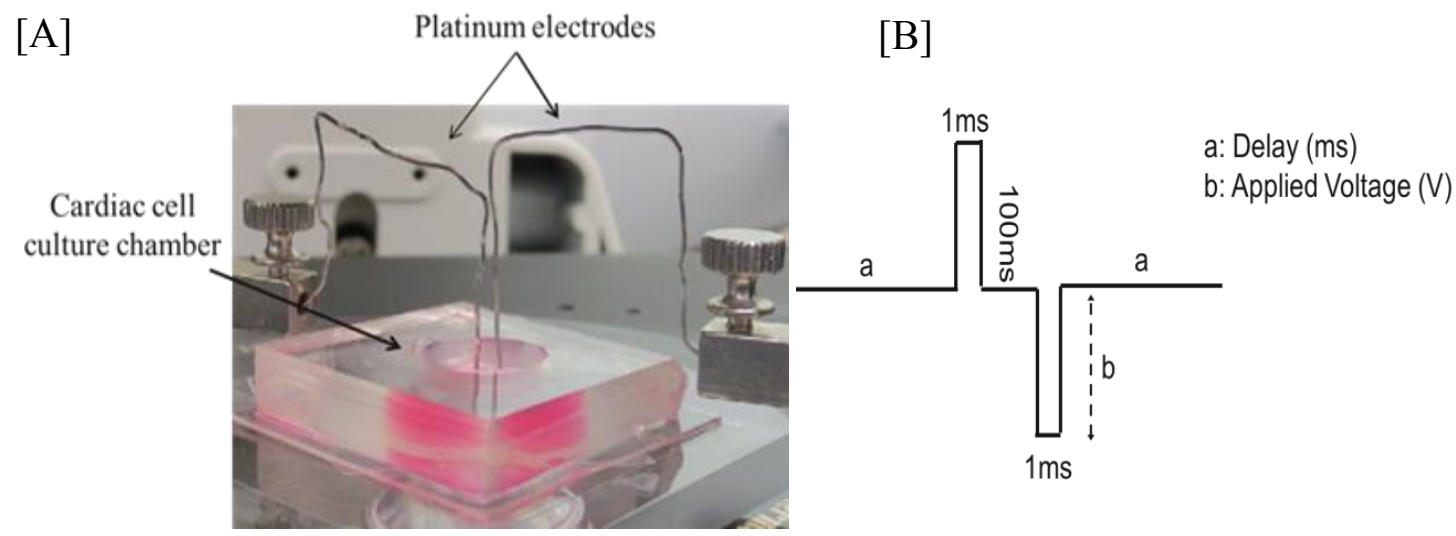

Figure 6.7: Electrical Pacing Setup System. [A] The image of the real pacing experiment, which was set on the stage of the microscope before pacing the tissue. [B] Diagram of the electrical stimulating, which was programed on the electrical pacing system.

The maximum pacing tissue beat rate was recorded while varying the threshold voltage. To find the maximum pacing rate, the voltage was increased from the lower to the higher voltage until the $\mathrm{BR}$ reached to $2: 1$ phase (BR was half of the maximum $\mathrm{BR}$ ). The threshold voltage was also determined. This voltage was the voltage at which the tissue started beating with the frequency of $2.0 \mathrm{~Hz}$. The results show that the CCCM tissues reached their maximum $\mathrm{BR}$ of $245 \mathrm{bpm}$, which is close to the $\mathrm{BR}$ of the in-vivo mature chick cardiac tissue (Fig. 6.8). In addition, the threshold voltage needed for the CCC tissues to reach the frequency of $2.0 \mathrm{~Hz}$ was lower than the threshold voltage inserted on the control samples. This result affirms that mechanical conditioning within the CCCM resulted in tissue maturation represented by more efficient cell-cell connections and higher maximal beat rates. 

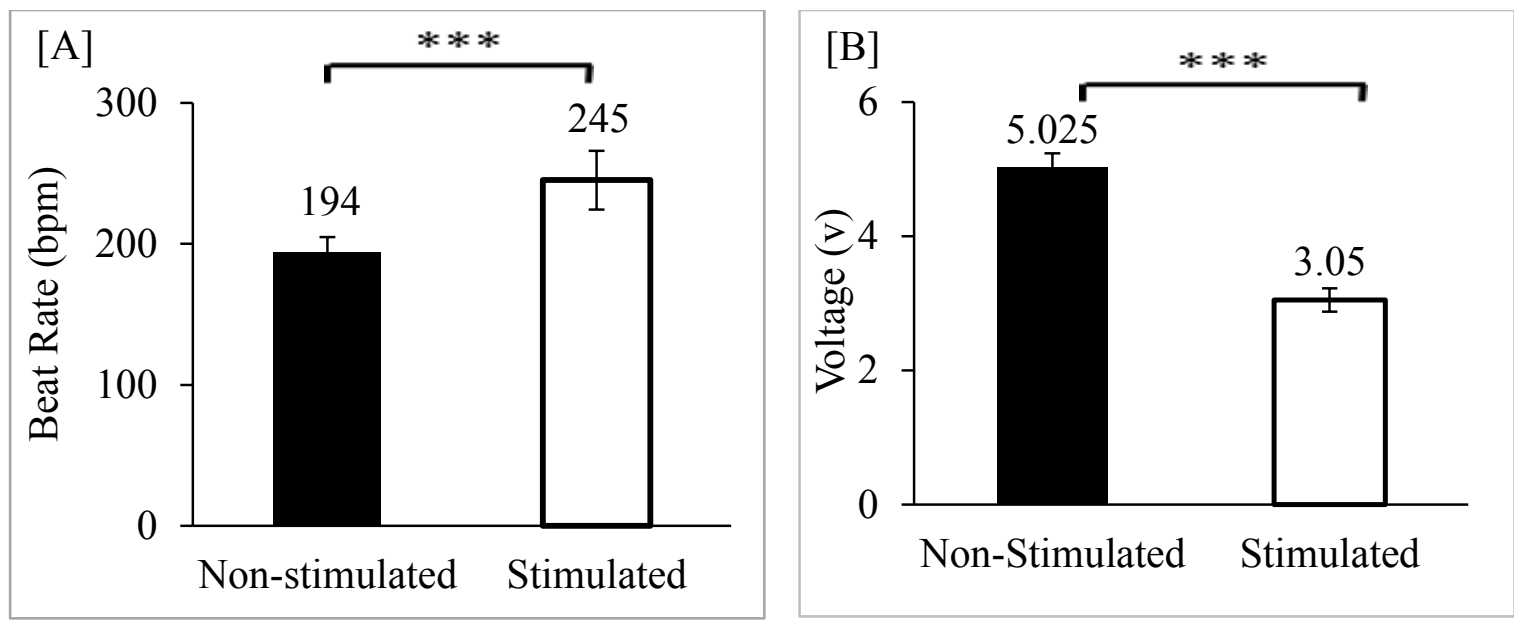

Figure 6.8: Pacing Electrical Stimulation Results. [A] The Maximum Pacing Beat Rate (bpm) between the non-stimulated (light blue) and the stimulated cardiac tissue group (red). [B] The Threshold Voltage (V) in corresponding to the maximum pacing beat rate for the non-stimulated and stimulated cardiac tissue groups. $\mathrm{N}=6$ with two-tailed significance set at $* * * \mathrm{P}<0.05$

\subsubsection{Cell contractility}

Movies of beating cardiomyocyte were recorded and analyzed to determine regional tissue strain during contraction. For an individual sample, 5 different locations on the movie were selected randomly for strain measurement. Lagrangian strain calculation for finitely small displacement was used as previously described by Bonet et al. ${ }^{146}$ Briefly, if $\mathrm{p}_{1}$ and $\mathrm{p}_{2}$ are two nearby points defined on the first frame, and $\mathrm{d}_{1}$ denotes the Euclidian distance between $p_{1}$ and $p_{2}$ at the first frame (i.e., reference frame), then $d_{j}$ denotes the Euclidian distance between these two points at time frame $j$, then, the engineering strain $\mathrm{S}$, at frame $\mathrm{j}$, is defined as:

$$
\mathrm{S}_{\mathrm{j}}=\left(\mathrm{d}_{\mathrm{j}}-\mathrm{d}_{1}\right) / \mathrm{d}_{1}
$$


A custom algorithm was written to estimate the Lagrangian strain. First, the peak displacement was determined by tracking two points based on their appearance features through the image frames and calculating the displacement between the two points over the image frames. The maximum displacement was then selected as the reference frame. Then, five pairs of points on the first (reference) frame were determined and their tracked pairs on the selected frame with maximum displacement. The Lagrangian strain for each pair of points was estimated using equation (8). Finally, the mean Lagrangian strain was calculated as expressed a \% shortening.

The results show that without the electrical pacing, the percentage shortening of the CCCM stimulated tissues was about $4.49 \pm 0.88 \%$, whereas controls were only at $2.73 \pm$ $1.8 \%$ (Fig. 6.9). The unpaired t-test shows no statistical significance between the shortening length of the control and the CCCM samples. However, when electrical pacing was applied, the shortening of the CCCM stimulated tissues increased to $5.52 \pm$ $0.88 \%$, whereas the shortening of the un-stimulated tissue only went up to $2.96 \pm 1.79 \%$. The statistical results showed significant difference with $* p<0.05$. These results suggest that, the mechanical stimulation enhances the shortening of the cardiac tissue, and this contractility increases further with the association of the electrical pacing applied on the tissue. 


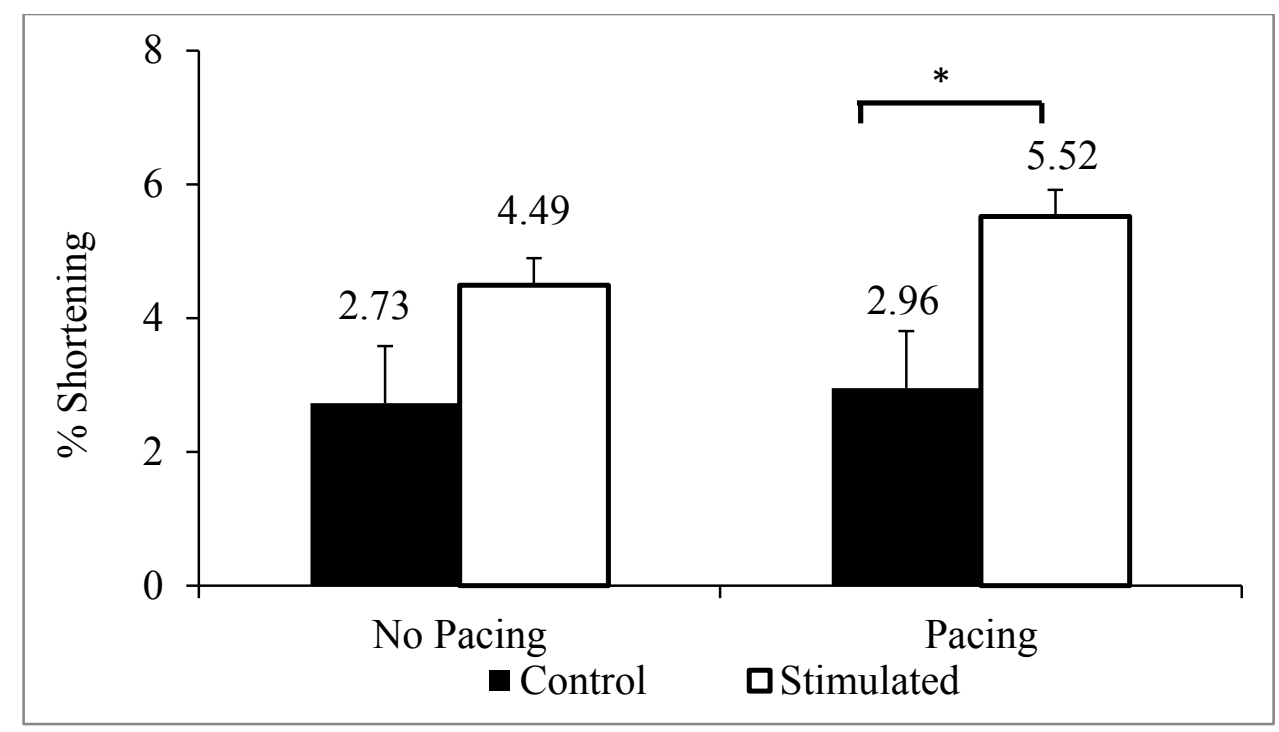

Figure 6.9: Graph of cardiomyocyte shortening with and without electrical stimulation: control (black) and the CCCM stimulated (clear) samples evaluated with and without electrical pacing. $\mathrm{N}=6$ with two-tailed significance set at $* \mathrm{P}<0.05$

\subsection{CARDIAC TISSUE RESPONSE TO $\beta$-ADRENERGIC STIMULATION}

\subsubsection{Introduction}

Isoproterenol (ISO) is a $\beta$-Adrenergic Agonist which binds to G-protein coupled $\beta$ Adrenergic receptors ( $\beta$-AR). The coupling of ISO/ $\beta$-AR activates adenylyl cyclase (AC). These activated AC catalyze the conversion of Adenosine triphosphate (ATP) into cyclic adenosine monophosphate (cAMP), followed by the activation of protein kinase A (PKA). After activated, PKA is able to phosphorylate L-type $\mathrm{Ca}^{2+}$ channel and phospholamban (PLB); as a result, increase the $\mathrm{Ca}^{2+}$ influx into the cytosol and enhance the activity of sarcoplasmic reticulum $\mathrm{Ca}^{2+}$ pump SERCA2a to transport $\mathrm{Ca}^{2+}$ from the cytosol into the sarcoplasmic reticulum (SR). Un-phosphorylated PLB inhibits the activity of the SERCA2a pump. Phosphorylating of $\beta$-AR via PKA prevents the coupling between ISO and $\beta$-AR and reduces the sensitization of $\beta$-AR to the agonist. ${ }^{54}$ In 
summary, ISO and $\beta$-AR play a significant role in the intracellular mediate signal transduction pathway by enhancing the $\mathrm{Ca}^{2+}$ influx into SR and the transportation of $\mathrm{Ca}^{2+}$ from the cytosol into the SR, therefore, contributes to regulate the heart function via contractility and frequency.

ISO affects the chick embryonic ventricle development as early as day $4^{\text {th }}$ (out of a total of 21 days of gestation before hatching) via the increase of contracting force of cardiac tissue. In contrast to the mature heart, ISO does not affect the early embryonic heart until the expression and coupling of functional $\beta$-AR. In addition to the positive chronotropic and inotropic effects of ISO stimulation, ISO treatment also has a toxic effect on developing and mature cardiomyocytes and can trigger cell death in cardiomyocytes from

a wide range of species. ${ }^{147-149}$ In adult hearts, ISO continues to enhance the rate of tension development and beat rate (BR) of the tissues. ${ }^{150}$ Our hypothesis that, if mechanical stimulation enhances the maturation of the cardiac tissue, in comparison to the control tissue group, the contractility and the BR of the stimulated tissue group must be increased in response to the ISO administration.

\subsubsection{Material and Methods}

Embryonic chick cardiac left ventricle cells at day 10 were isolated, seeded, and experimented as mentioned in the previous sections. After being cultured for 4 days with mechanical stimulation within the CCCM system, cardiac tissues were removed from the system and incubated in a static condition at $37^{\circ} \mathrm{C}$ for another 3 hours before applying ISO to the tissue. After $3 \mathrm{~h}$, samples were brought to a microscope for analysis. Different 
concentrations of ISO $(0.5 \mu \mathrm{M}, 1 \mu \mathrm{M}, 5 \mu \mathrm{M}, 10 \mu \mathrm{M}$, and $50 \mu \mathrm{M})$ (Sigma Aldrich, I6504) in warmed fresh medium were applied respectively to the tissues. An electrical hot plate was used to keep the temperature of the tissues and medium at $37^{\circ} \mathrm{C} . \mathrm{BR}$ before and after administrations were reported; and contractile movies were made within 5 minutes of each ISO administration. Then, a brief washing with warmed fresh culture medium was performed before adding a new higher dose of ISO to the tissue. Contractile movies were analyzed using MATLAB software (MathWorks, Naticks, MA) to determine the percentage shortening for tissue contractility.

\subsubsection{Results}

The effect of ISO on the BR and contraction of the constructs cultured in the mechanically active CCCM system and in static conditions were observed. The BRs before and after each ISO application were recorded. The BR of the tissue before the $0.5 \mu \mathrm{M}$ ISO solution added to the tissue was used as the referent BR to determine the percentage change in BR under the effect of ISO administrations (Fig.6.10). The results show that the BR of the CCCM group increased in response to increasing doses of ISO to a maximal response at $10 \mu \mathrm{M}$ ISO, then declined. In contrast, the BR of the control group decreased in response to increasing doses of ISO above $0.5 \mu \mathrm{M}$. This result indicated that the ISO produced a toxic response in static (unconditioned) chick cardiomyocytes. However the CCCM stimulated group had positive chronotropic responses to ISO, consistent with CM maturation. 


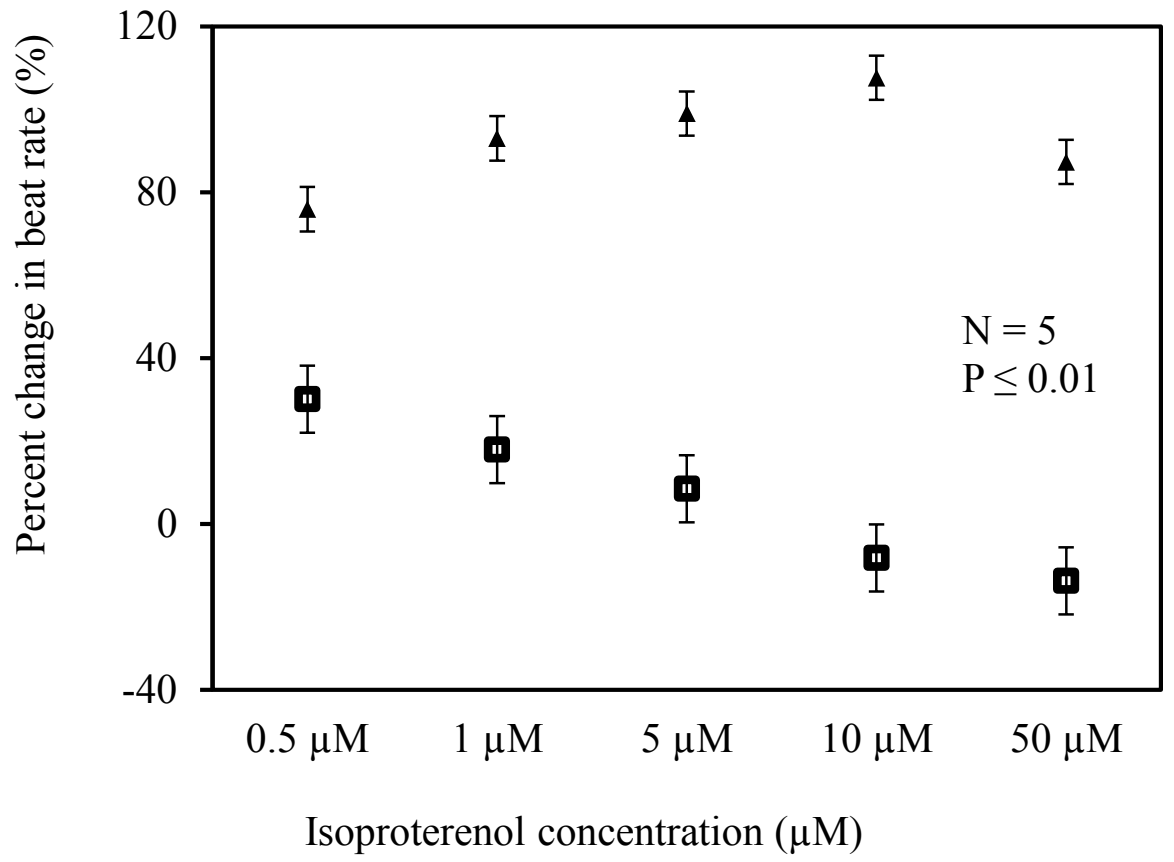

$\triangle$ Stimulated $\quad \mathbf{0}$ Control

Figure 6.10: Plot represents the percentage change in beat rate of the cardiac tissue under the effects of Isoproterenol. While the control group showed cellular toxicity with increasing ISO concentration, the beat rate of the stimulated group increased and reached its peak at $10 \mu \mathrm{M}$ ISO. The horizontal line is the base line corresponding to the referent beat rate recorded before the ISO applications. Five experiments were performed.

Cardiac contractility with and without ISO was also examined. Movies of each ISO application were made and maximum contraction was chosen to determine tissue shortening. Shortening was calculated as described in the section 6.4.3. With ISO applications contractility of the stimulated tissues significantly increased versus the unstimulated group, to $7.55 \pm 0.78 \%$ versus $4.52 \pm 0.43 \%$, respectively (Fig. 6.11). In response to ISO, contractility increased $67 \%$ in the conditioned group versus $36 \%$ in the 
unconditioned group. These results suggest that mechanical stimulation may have accelerated $\mathrm{CM}$ maturation as measured by reduced toxicity and increased $\mathrm{BR}$ and shortening in response to ISO stimulation.

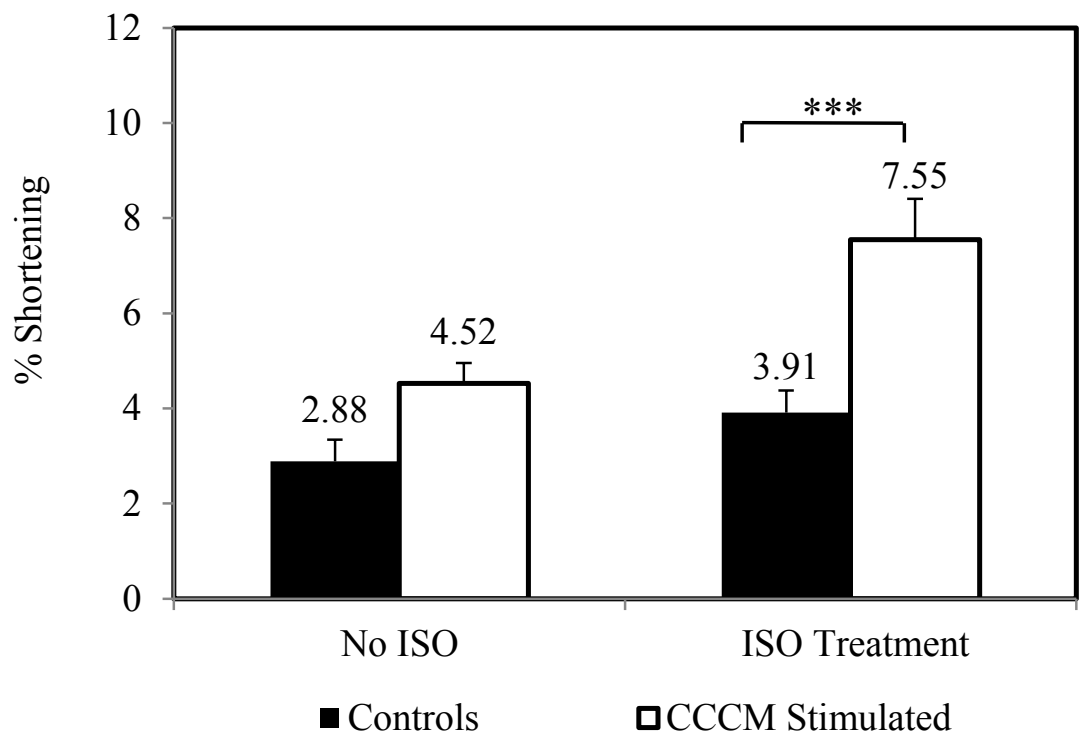

Figure 6.11: Changes in the cardiomyocyte contraction with and without the influence of ISO: controls (black) and CCCM stimulated (clear) constructs. Measurements were made at 3 different locations on each sample. $\mathrm{N}=6$ different paired experimental sets. Two tailed significance was set at $* * * \mathrm{p} \leq 0.001$.

\subsubsection{Discussion}

Catecholamine affects mechanical performance of the heart tissues through the activation of $\beta$-AR; and the cardiovascular function is controlled primarily on this adrenergic signaling pathway. ${ }^{52-54}$ Under normal physiological conditions, catecholamine induces positive inotropic and lusitropic responses in the heart tissue through $\beta 1$-AR-activated pathway. Once stimulation of $\beta 1$-ARs via ISO binding occurs, cAMP accumulation 
rapidly increases which indirectly promotes the phosphorylation of PLB, and enhances the cardiac contractility. Out of their subtypes, subtype $\beta 1$-ARs exhibits dominantly when cardiomyocytes become more mature under normal physiological condition. ${ }^{53,56}$ This dominant $\beta 1$-ARs, therefore, induces positive inotropy and lusitropy via heart rate and contractility. Under the abnormal physiological condition of the heart, or failing heart, $\beta 1$-ARs decreases, but $\beta 2$-ARs increases, causing cardiomyocyte death. ${ }^{56}$

After adding the ISO to the tissue, the CCCM conditioned constructs yielded a higher percentage of contraction compared to the control ones; and the BR of the conditioned tissue kept increasing until a $10 \mu \mathrm{M}$ ISO solution $(2.5 \mathrm{mg} / \mathrm{ml})$ was added. However, for the controls, the concentration of ISO higher than $10 \mu \mathrm{M}$ created negative inotrope and reduced BR on the cardiac tissue, and finally the cardiac tissue stopped beating This result is similar to the result found in the early studies from Serverin, ${ }^{151}$ indicating that these CM undergo cell damage and death if a higher concentration of ISO is applied. These results state that, compared to the static cultured control group, the CCCM stimulated construct has a higher response to the $\beta$-AR, and at the same time, has a higher resistance against the cellular toxic effect of ISO, hence, is less likely to undergo cell damage and death. Higher response and higher resistance may be the result of higher cell-cell connection, more maturity, or more population. In conclusion, mechanical stimulations of embryonic $\mathrm{CM}$ in the CCCM resulted in a more mature ISO response, consistent with the maturation of $\beta 1$-adrenergic signaling. 


\subsection{SELECTED CARDIOMYOCYTE GENE AND PROTEIN EXPRESSIONS IN RESPONSE TO MECHANICAL CONDITIONING}

\subsubsection{Introduction}

From previous results, embryonic chick cardiac tissues cultured in this CCCM system showed a higher proliferation rate, better alignment, and more maturation via BR and contractility. This section focusses on the effect of mechanical stimulation on proteins that play a critical role in the beating rate and contractility of the heart tissue. Along with the $2 \mathrm{D}$ constructs (CCCM stimulated and the control ones), ventricle tissues at day 16, cell suspension from day $16^{\text {th }}$ (same day harvesting the $2 \mathrm{D}$ constructs), and $3 \mathrm{D}$ engineered cardiac tissues (ECT) harvested at culture day 5 (equivalent to day 16 chick ventricular cells) were also collected for selected gene expression and protein synthesis analysis.

As markers of CM maturation and function, sarcoplasmic reticulum calcium ATPase $2 \mathrm{a}$ (SERCA2a), phospholamban (PLN), Troponin T (TnT), and Alpha and Beta-Myosin Heavy Chain $(\alpha-, \beta-\mathrm{MHC})$, and total protein were analyzed in control and conditions tissues.

SERCA2a and PLB are essential proteins that regulate calcium cycling inside the cardiomyocytes. These genes are involved in regulating the contraction-relaxation of the heart muscle. Un-phosphorylated PLB binds to SERCA2a, prevents $\mathrm{Ca}^{2+}$ binding to SERCA2a, and acts as an endogenous inhibitor of SERCA2a. Phosphorylated PLB (pPLB) releases this inhibitory effect, making the affinity site to $\mathrm{Ca}^{2+}$ on SERCA2a more 
apparent. This activated SERCA2a then transports calcium against the gradient from cytoplasm back to sarcoplasmic reticulum (SR) ${ }^{58}$ As the result, the rate of calcium ions influx back to the SR increases, promoting the muscle relaxation prior to the next cycle of calcium release and myofiber contraction. Therefore, SERCA2a has a central role in regulating $\mathrm{Ca}^{2+}$ cycling between the SR and myofibers and a direct impact on $\mathrm{CM}$ contractility. The regulation of SERCA2a activities plays a critical factor in the contractile function and heart disease. ${ }^{152} 32$

$\mathrm{TnT}$ is the protein binding with tropomyosin and involved in the regulation of thin filament function and the interaction between the thin and thick filament during myofiber shortening. ${ }^{50}$ Abnormalities of this TnT gene are associated with sudden death in cardiac patients. Myosin heavy chain (MHC) is the major sarcomeric protein expressed in heart tissue during development and adulthood and regulates mechanical performance of the heart, especially muscle contractility. ${ }^{64-66}$ Alpha and Beta MHC are the two important isoforms of the MHC found in the heart tissue. $\beta-\mathrm{MHC}$ isoform is expressed mainly during embryogenesis or heart diseases. $\alpha-\mathrm{MHC}$ isoform exists dominantly in the adult heart tissue and inhibits the expression of $\beta$-MHC. The ratio of $\alpha$-MHC to $\beta-\mathrm{MHC}$ expression is also seen in species with rapid or slow beating rates ${ }^{67}$

\subsubsection{Methods}

\subsubsection{Cell suspensions and 3D ECT constructs}

Cell suspension from ventricular cardiomyocyte tissues from day 16 were also isolated using GentleMACS ${ }^{\mathrm{TM}}$ Dissociator (MACS Miltenyi Biotec, Auburn, CA), pre-plated, and 
rotated before being collected for cDNA and proteins. At the same time, ventricular tissues from day 16 were also collected for cDNA and proteins.

To create 3D ECT constructs for PCR, the isolated embryonic cardiomyocyte clusters ( $3.0 \times 10^{4}$ clusters) were also mixed with collagen solution $(0.667 \mathrm{mg} / \mathrm{ml})$ and seeded in the TT-4001C Tissue Train ${ }^{\mathrm{TM}}$ plates (Flexcell ${ }^{\circledR}$ International Corporation, HillSborough, NC) using Flexcell FX5X Tension system until the collagen solution was cured (about $1.5 \mathrm{~h}$ vacuumed, $37^{\circ} \mathrm{C}, 5 \% \mathrm{CO}_{2}$ ). Then the constructs were cultured under the static condition for 5 days prior to analysis for PCR.

\subsubsection{Real time PCR (qPCR) for gene expression}

Total RNA was extracted from the cardiac constructs, cell suspensions, or ventricles, using the Trizol isolation method. Briefly, the sample was homogenized in $1 \mathrm{ml}$ Trizol three times $(7 \mathrm{sec} / \mathrm{each})$ in ice. After RNA isolation, cDNA was then transcribed via the RNeasy mini kit (QIAGEN). The concentration of cDNA was measured using the NanoDrop 2000 spectrophotometer (Thermo Scientific, Waltham, MA). The real time PCR for each target gene was then performed in triplicate. The GAPDH primer was included as the housekeeping gene. SERCA2a, $\alpha$ and $\beta$ MHC, TnT, and PLB were the interested genes chosen for these PCRs. For data analysis, the relative quantification of each target gene in each group was normalized to GAPDH. The fold change of each interested gene was determined by $\Delta \Delta \mathrm{C}_{\mathrm{t}}$ where the calibrating sample is the control construct. 


\subsubsection{Western blotting for protein synthesis}

The end of the experiment, total protein of each sample was collected using the Complete-Lysis M solution (Roche Diagnostics, Indianapolis, IN). The protein concentration was then determined, using the NanoDrop 2000 Spectrophotometer. The target proteins were separated using 10\% SDS-PAGE and transferred to Immobilon ${ }^{\mathrm{TM}}$-FL membranes (Millipore Corporation, Bedford, MA). After being blocked with 5\% non-fat milk, the membrane was probed with different primary antibodies including antiSERCA2a (1:500) (LSBio, Seattle, WA), anti $\alpha$-MHC (1:150) (Santa Cruz, Dallas, TX) and $\beta$-MHC (1:500) (Bioss, Woburn, MA), anti-Actinin (1:500) (Abcam, Cambridge, MA), anti TnT (1:1000) (Sigma, St. Louis, MS) and anti $\beta$-actin (1:3000) (Cell Signaling, Beverly, MA). The secondary antibody was Cy $3{ }^{\circledR}$ goat anti-Rabbit IgG (1:500) (Life Technologies $^{\mathrm{TM}}$, Grand Island, NY) and $\mathrm{Cy} 5^{\circledR}$ goat anti-mouse IgG1 (1:500) (Abcam, Cambridge, MA). The labeled bands representing the target proteins were visualized via the Typhon 9400 system. NIH Image J software program was used for densitometry.

\subsubsection{Results and discussions}

\subsubsection{Gene expression}

Figure 6.12 and figure 6.13 show that compared to the level of gene expression in the embryonic chick ventricle, gene expression decreased progressively from cells suspension to 2D CCCM culture for all three genes: SERCA2a, $\alpha-\mathrm{MHC}$, and $\beta$-MHC. The gene expression on $2 \mathrm{D}$ constructs, both the controls and the CCCM stimulated samples, was much lower compared to the heart ventricles and cell suspension on day $16^{\text {th }}$, as was shown in a previous study. ${ }^{93}$ When compared, the $2 \mathrm{D}$ constructs together, 
gene expressions between the control and the CCCM group showed no significantly statistic increase or decrease. The $\mathrm{p}$ values were greater than 0.05 . However, gene expressions of these genes were partially restored with the 3D ECT constructs especially with PLN, $\alpha$-MHC, and $\beta$-MHC genes .
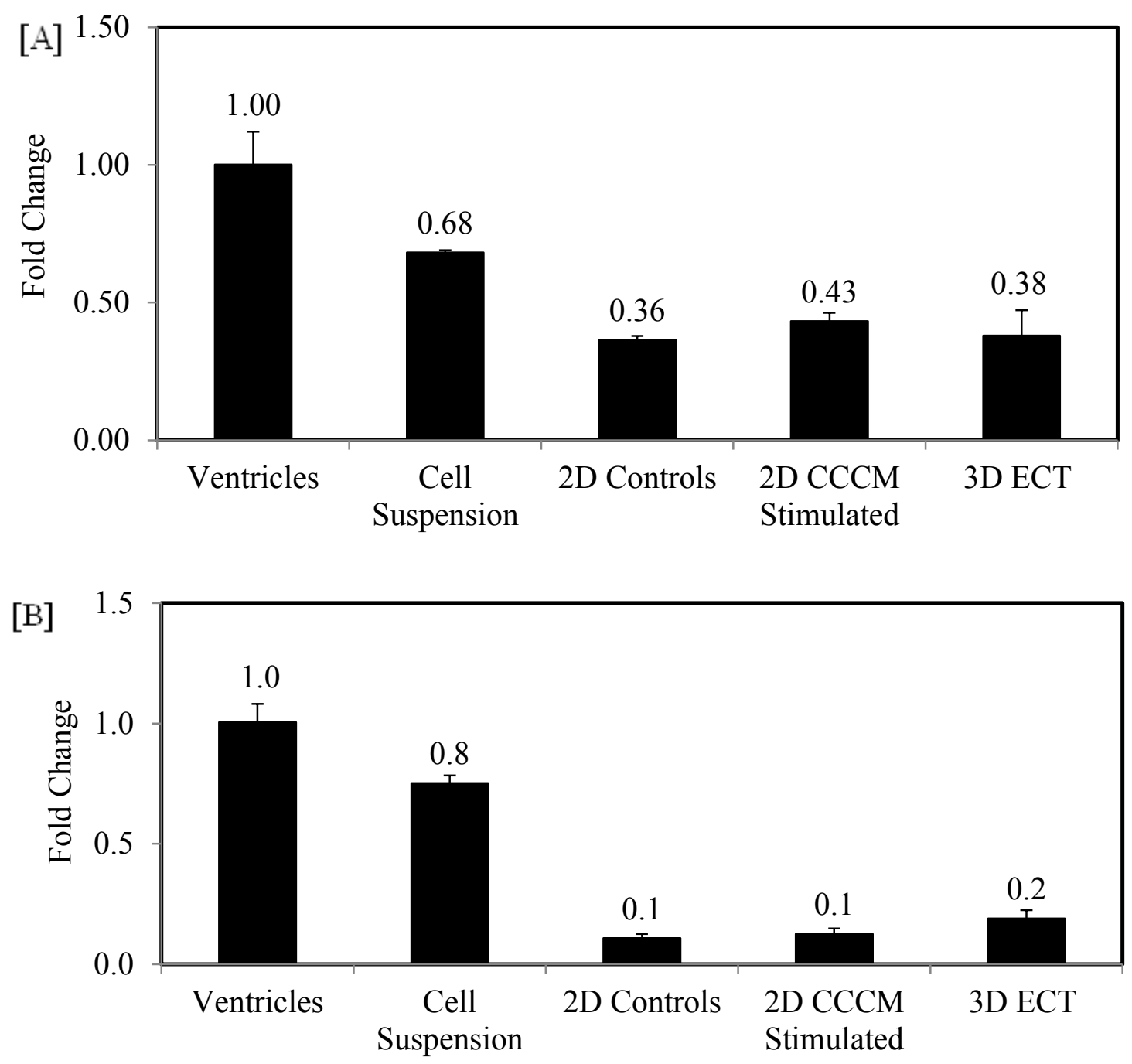

Figure 6.12: Plots of fold changes in gene expression with ventricular gene expression set as the control state. [A] SERCA 2a gene expressions, [B] PLN gene expression. All samples were collected on day $16^{\text {th }}$ (out of 21 days of gestation). $N \geq 3$ and $p>0.05$. 


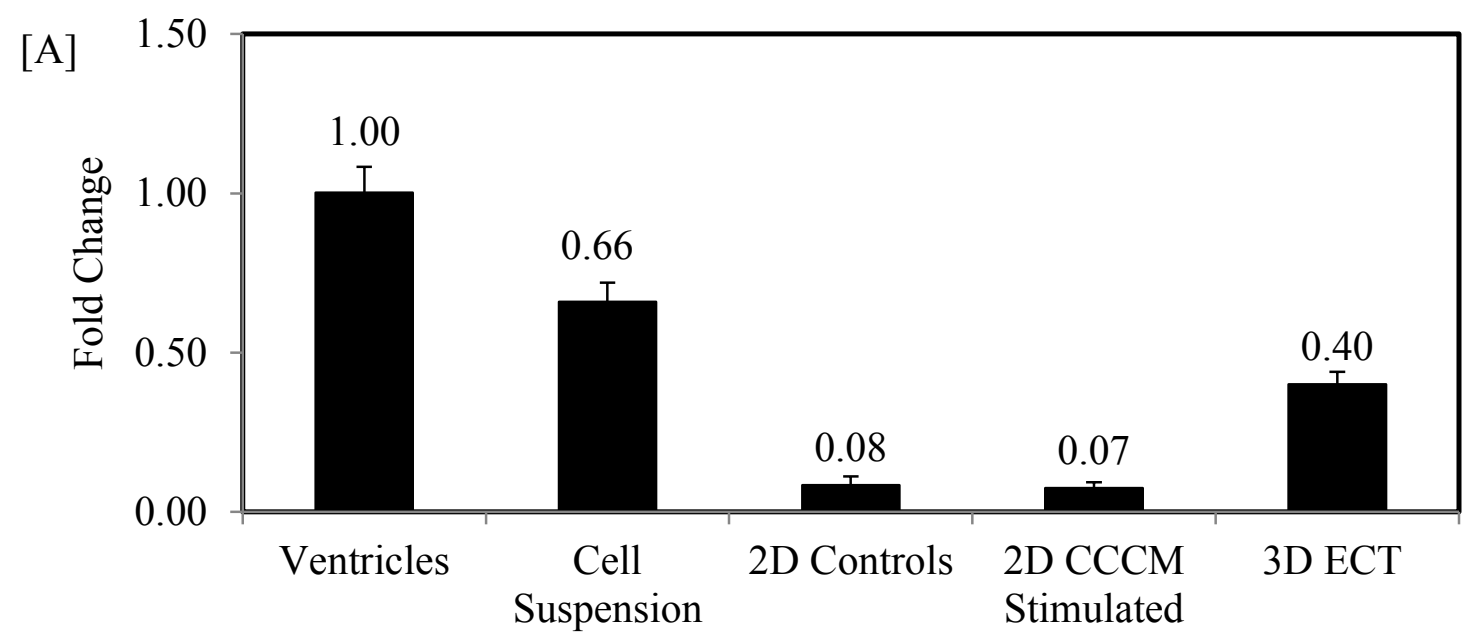

[B]

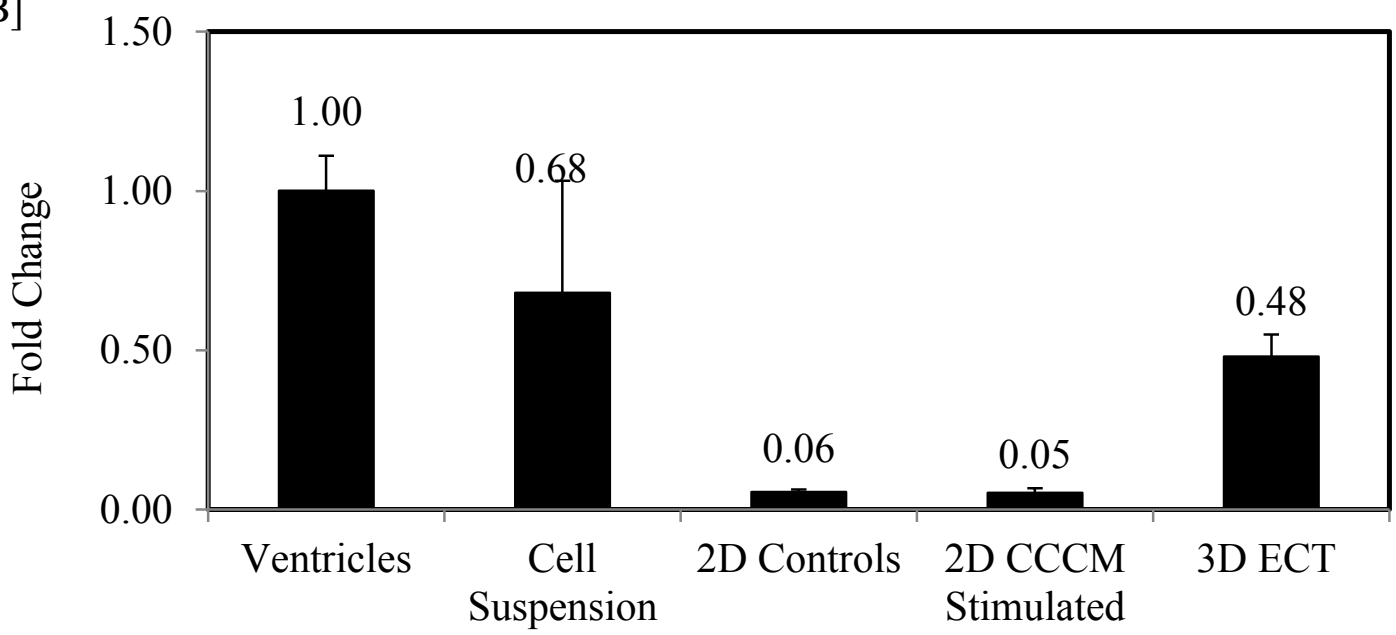

Figure 6.13: Plots of fold changes in gene expression with ventricular gene expression set as the control state. [A] $\alpha-\mathrm{MHC}$ gene expressions and [B] $\beta$-MHC gene expressions. All samples were collected on day $16^{\text {th }}$ (out of 21 days of gestation). $\mathrm{N} \geq 3$ and $\mathrm{p}>0.05$.

Figure 6.14 shows the total RNA synthesis obtained from 2D cardiac construct groups: the control and the stimulated ones. From the plot, the embryonic chick cardiac tissues stimulated yielded more RNA $(1803 \pm 218 \mathrm{ng} / \mu \mathrm{l})$ than the tissues cultured under the static condition $(1186 \pm 102 \mathrm{ng} / \mu \mathrm{l})$; which was about 1.5 time higher. 


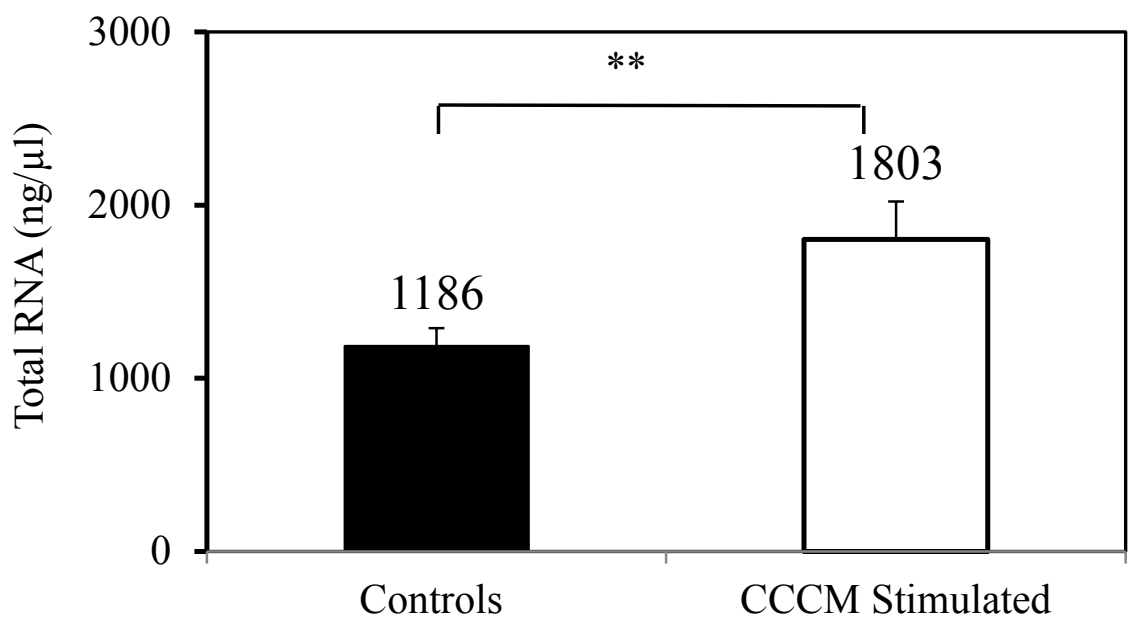

Figure 6.14: Total RNA obtained from $2 \mathrm{D}$ embryonic chick cardiac tissues. Total samples $\mathrm{N}=3, * * \mathrm{p}<0.05$

\subsubsection{Protein Synthesis}

More than 10 samples in pair (controls vs the CCCM stimulated groups) were used to determine the total protein in each group. The total protein in the CCCD stimulated constructs significantly increased $(97.15 \pm 4.97 \mathrm{mg})$ versus total protein in the $2 \mathrm{D}$ controls (71.52 $\pm 4.14 \mathrm{mg})$ (Fig. 6.14)

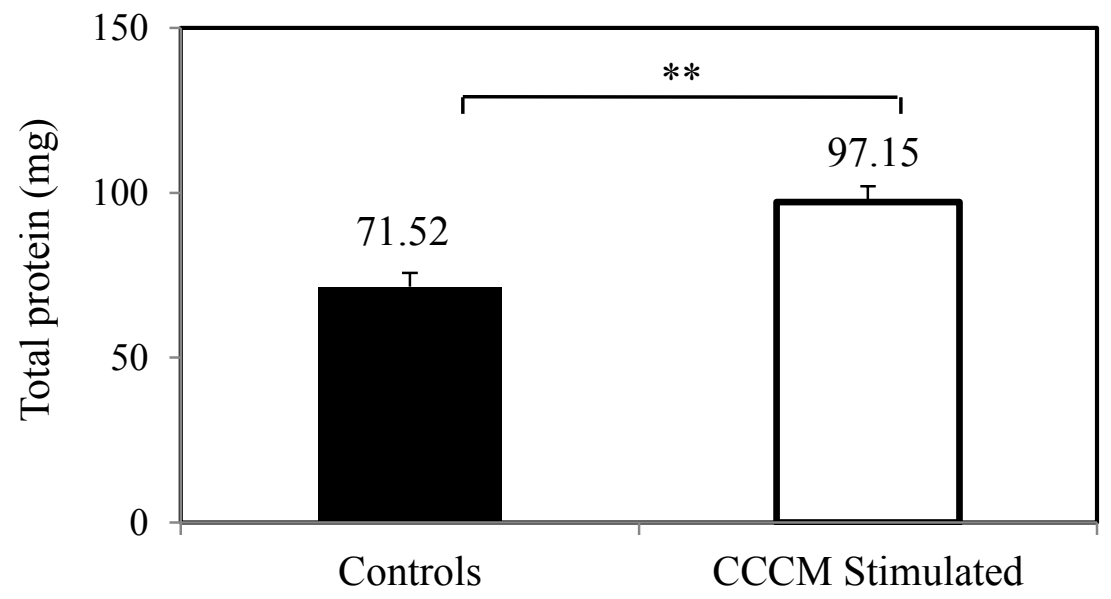

Figure 6.15: Total proteins of the $2 \mathrm{D}$ cardiac tissue constructs. $\mathrm{N} \geq 12$ two tailed significance was set at $* * \mathrm{p}<0.005$. 
Five different CM proteins (SERCA2a, $\alpha$ - and $\beta$-MHC, $\alpha$-Actinin, and TnT) were selected for the Western Blot (Fig. 6.16). Figure 6.17 and figure 6.18 show the target proteins collected from different conditions. Proteins SERCA2a, TnT, and $\beta$-MHC from the cell suspension were much lower than those in the ventricular tissues. Interestingly, the amount of $\alpha$-Actinin protein almost doubled after cell isolation from ventricular tissue. As noted in the previous study, protein levels decreased in response to 2D culture. However, when mechanically conditioned in the CCCM system, protein levels were modestly increased, except for myosin heavy chain proteins.

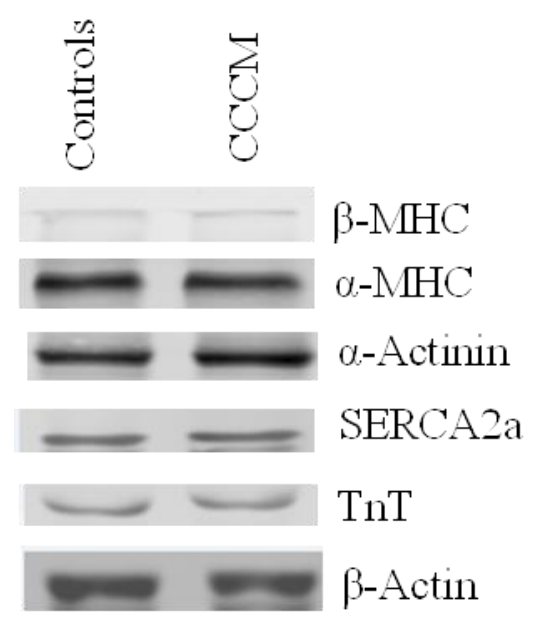

Figure 6.16: Images of Western Blot bands of the interested proteins in the $2 \mathrm{D}$ control and CCCM stimulated groups 
[A]

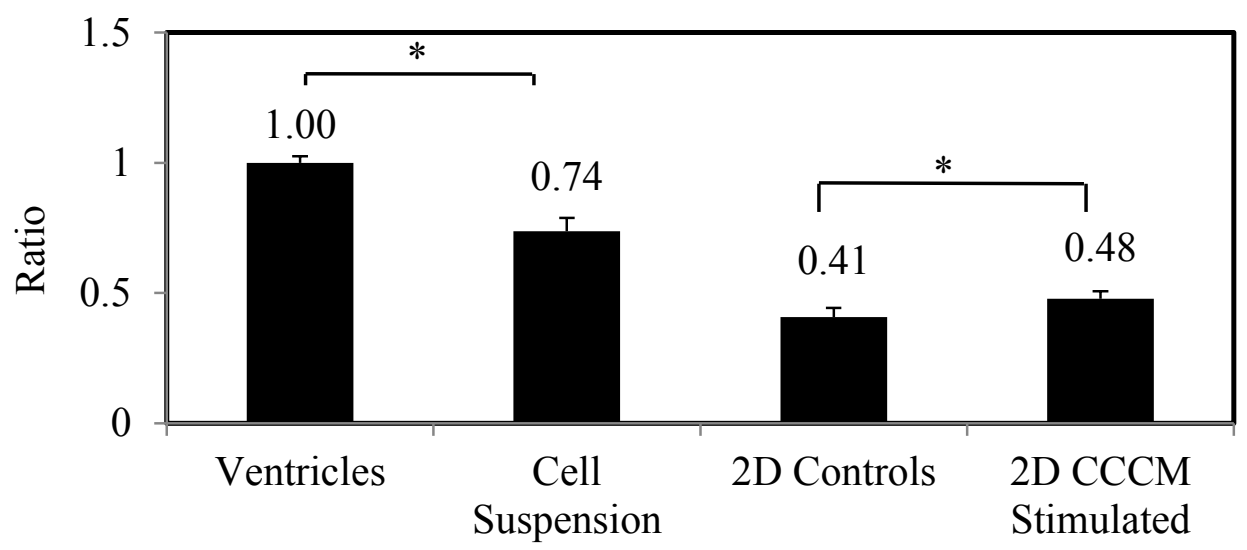

$[\mathrm{B}]$

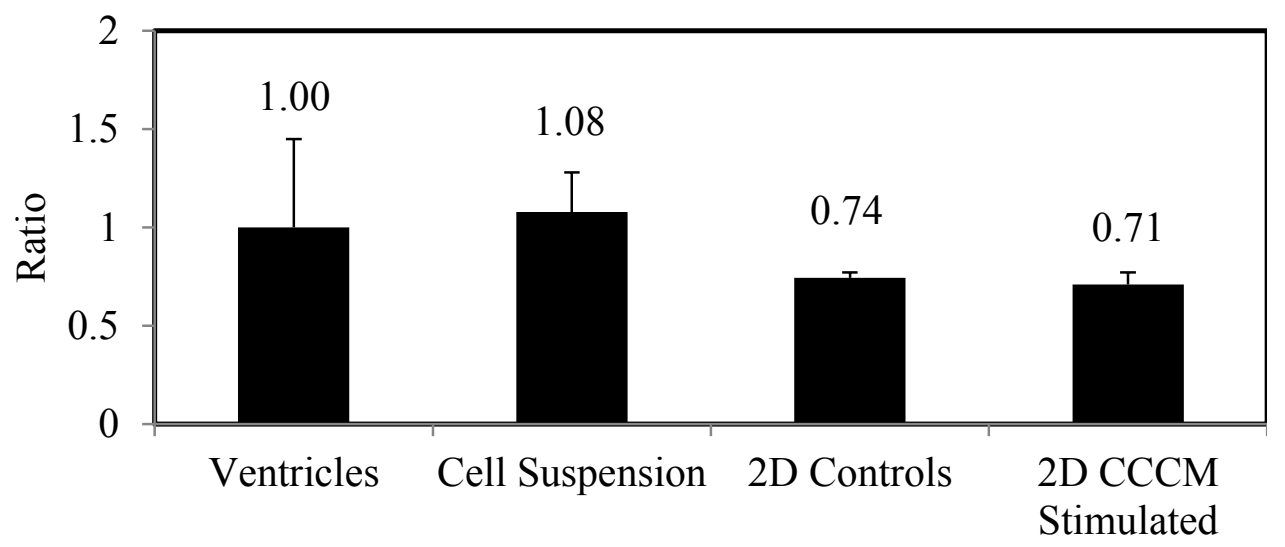

[C]

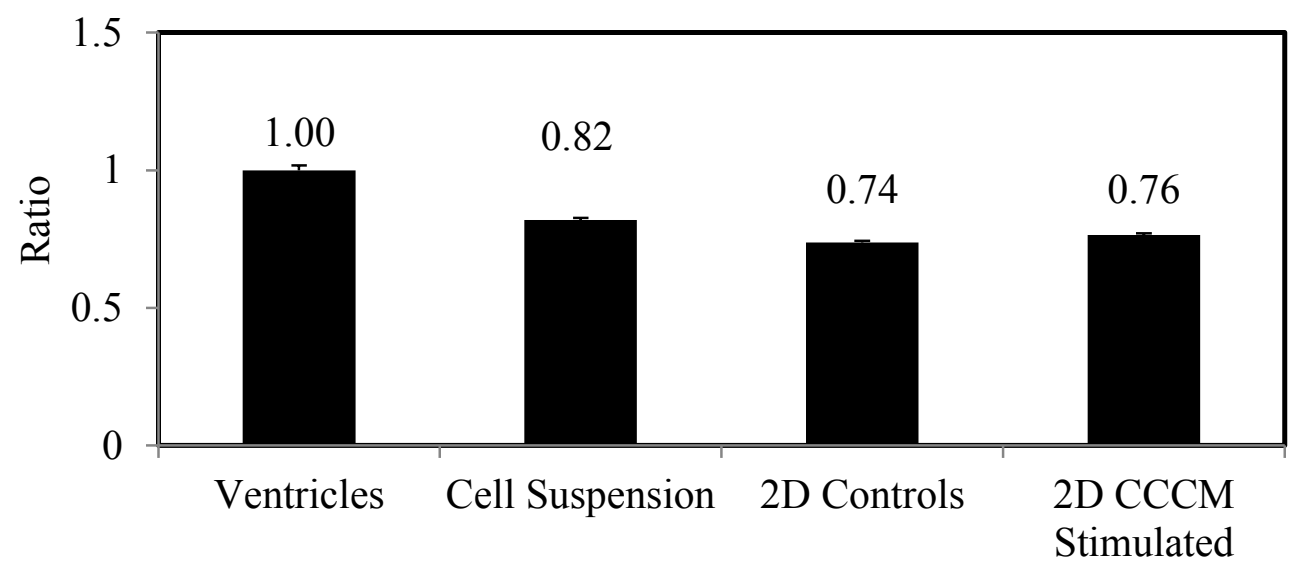

Figure 6.17: Graphs of SERCA2a, $\alpha$ - and $\beta$-MHC proteins normalized by $\beta$-Actin. [A] SERCA2a protein, [B] $\alpha$-MHC protein, and [C] $\beta-\mathrm{MHC}$ protein. $\mathrm{N} \geq 3$ and two tailed significance was set at $* \mathrm{p}<0.05$. 
[A]

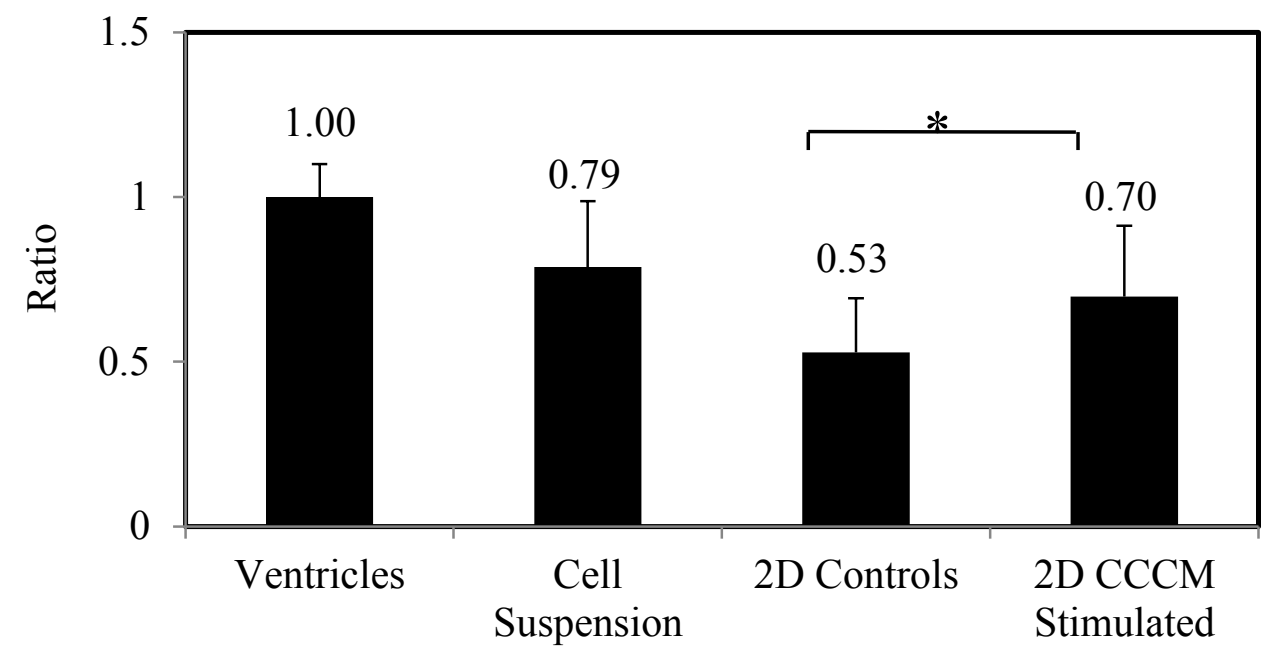

[B]

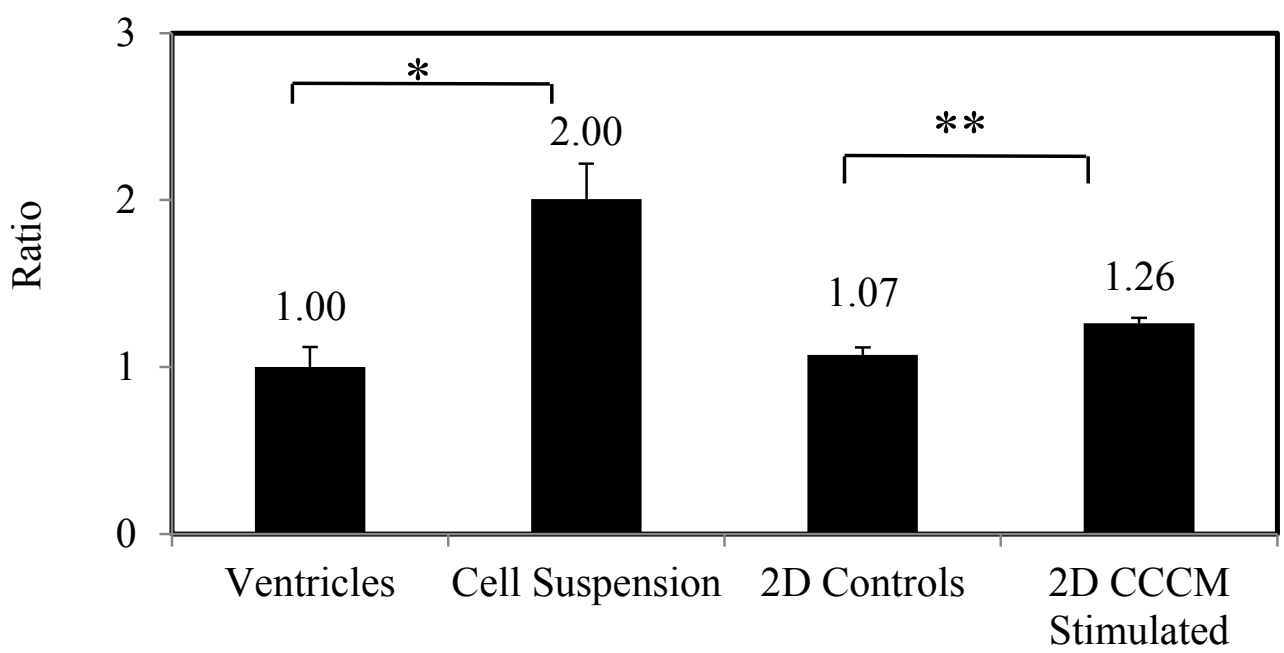

Figure 6.18: Graphs of $\operatorname{TnT}$ and $\alpha$-Actinin proteins normalized by $\beta$-Actin. [A] TnT protein and $[\mathrm{B}] \alpha-$ Actinin protein. $\mathrm{N} \geq 3$. SEM was used for error bars; and two tailed significance was set at $* \mathrm{p}<0.05$ and $* * \mathrm{p}<0.005$.

\subsubsection{Discussion}

During cell isolation, cell-cell contacts are disrupted and extracellular matrix is destroyed, causing cells to lose the extra cellular network connections that influence many cellular functions, including calcium handling and contractility. Consistent with the 
negative impact of cell isolation is the disruption of gene expression and protein synthesis of contractile relating proteins. ${ }^{93}$ As a result, gene expression and protein synthesis of the $\mathrm{Ca} 2+$ regulating protein (SERCA2a) and contractile proteins ( $\beta$-MHC) were lower in the cell suspension group than in the ventricle group. However, these results also demonstrate that transcriptional and translational processes still occur and that viable cardiomyocytes recover and mature in the CCCM. The protein $\alpha$-MHC obtained from the cell suspension was higher than those in the ventricular tissues, but was not statistically significant. However, when these embryonic cardiac cells were cultured under 3D, gene expressions of $\alpha-\mathrm{MHC}, \beta-\mathrm{MHC}$, and PLN were augmented, demonstrating that $3 \mathrm{D}$ culture is necessary for the cardiac tissue to fully regulate the transcriptional processes. These results are similar to the results done by L.Khait and R.K.Birla group with neonatal rat tissues. ${ }^{93} 2 \mathrm{D}$ constructs are compacted, cells are laid on top of each other; therefore, limiting their ability to arrange their orientation and their potentials to create their own cellular matrix as well as to access their nutrients. 3D constructs allow cells more space to rearrange their orientation, create their own cellular matrix, and build up their connection among cells. Nutrients are easy to access. Therefore, when cells were cultured under 3D condition, their gene expressions are nearly restored to levels found in the 3D native ventricular tissues even though all these 3D ECT constructs in this experiment were cultured under the static condition.

In contrast to other $\mathrm{CM}$ associated proteins, the protein synthesis of $\alpha$-Actinin protein doubled in the short time from ventricle tissue to cell suspension. The amount of Alphaactinin was also higher than those in the ventricular tissues. In cardiac cells, $\alpha$-actinin 
protein is the cytoskeletal actin-binding protein to stabilize the contractile apparatus. ${ }^{42,153 \text {, }}$ ${ }^{154}$ Once the extra-cellular network was disrupted during the isolation, this protein synthesis may be augmented due to the greater need in order to maintain the integrity of cardiac function and stabilize the shape of the cells.

For 2D constructs, compared to the control unstimulated group, SERCA2a protein synthesis in the stimulated tissue increased. However, the result from gene expression showed no statistically significant change in the SERCA2a gene expression including the 3D ECT constructs. In the cardiac cells, the sarcoplasmic reticulum where $\mathrm{Ca}^{2+}$ is stored during the relaxing phase becomes functional only around birth and embryonic chick CM are known to have immature SR; ${ }^{155}$ hence, it is understandable to show no significant difference in SERCA gene expression. Further, the level of PLN in the CCCM tissues was not significantly increased, compared to the static culture tissue, indicating that cells did not undergo negative regulation in response to mechanical stimulation within the CCCM system. In addition, the amount of total protein obtained from the conditioned group was higher than the control one. All these results affirm that mechanical stimulation during embryogenesis mainly enhanced proliferation rate rather than increase gene expression for SERCA. Understanding these biomechanical responses of the cardiac embryonic cells will be important for engineering myocardial tissue studies.

The two cardiac MHC isoforms reciprocally shift their expression depending on the developmental stage and physiological condition of the heart. Before birth, $\beta$-MHC is the main $\mathrm{MHC}$ isoform in the ventricle. After birth, $\beta$-MHC reduces dramatically but $\alpha$ - 
MHC increases significantly and becomes the main MHC isoform. The reversal of these 2 MHC isoforms is seen under heart failure diseases or in an aging heart in which $\beta$ MHC is dominant. Our results showed no significant change of the $\alpha$ - and $\beta$-MHC gene expression and the protein synthesis within 2D construct groups. First, no increase in the $\beta$-MHC gene expression indicates that tissues undergoing mechanical treatment in the CCCM system may become more mature and are not overstressed, which was supported by the PLB gene expression level above. Second, this study was performed during the embryonic and fetal stage, five days before hatching, indicating that the $\alpha$-MHC expression will not be dominant yet. Third, the expression of these isoforms is controlled by thyroid hormone T3. ${ }^{64}$ Applying mechanical stimulation at an early stage of cardiac tissue development may not be enough to promote $\alpha$ - and $\beta$-MHC gene expression, as well as protein synthesis. In fact, the gene expressions and the reciprocity of these MHC isoforms are controlled by developmental stages and thyroid hormone T3. ${ }^{64,} 156$ Therefore, for further maturation of the tissue, besides mechanical conditions, the thyroid hormone needs to be included to generate more MHC, especially when dealing with embryonic cardiac tissue engineering for tissue transplant.

\subsection{CONCLUSION}

We have demonstrated that early mechanical stimulation during embryogenesis is needed for tissue proliferation, maturation, responses to the $\beta$-adrenergic agonist, and protein synthesis. Mechanical stimulations also help to maintain the calcium regulation inside the cells. These results prove that CCCM system is a novel system to insert the in-vitro mechanical stimulation on cardiac tissue; therefore, holds significant promise for 
generation of functional cardiac patches for replacement of injured cardiac tissue. 3-D cell culture in CCCM with incorporation of thyroid hormone and mechanical stimulation may be necessary for cardiac tissue engineering. 


\section{CHAPTER 7}

\section{CONCLUSION}

\subsection{SUMMARY}

CVD continues to be a major global healthcare dilemma. Current treatment options, though beneficial, have significant limitations. In order to address these issues and provide new treatment options, a better understanding of the molecular pathophysiology of CVDs is necessary. Cellular level models provide the best platform for discovery based studies. However, current approaches for cardiac cell culture fail to accurately replicate the mechanical loading conditions seen in-vivo as a consequence of normal hemodynamic loading and unloading. Available techniques address mechanical loading on an individual basis, but to date, no system that accurately replicates the in-vivo mechanical loading environment exists. This has resulted in cell-level studies being conducted in environments with little or no physiological relevance.

The CCCM system was designed and developed to culture various cardiac cells in a physiologically relevant environment by replicating the mechanical loading conditions generated as a consequence of normal hemodynamic loading and unloading. Various variables like cyclic pressure, strain and shear can accurately be replicated in a pulsatile fashion similar to conditions experienced in the left ventricle of a heart. Characterization 
studies show that mechanical loading profiles associated with normal and various disease conditions of the human heart, including heart failure, hypertension, hypotension, bradycardia and tachycardia can be accurately replicated over extended periods of time. Finally, proof-of-concept studies using primary chick embryonic ventricle cardiomyocytes confirms the ability of this system to generate in-vivo like mature cardiac tissue cultured within this platform in phenotype, function and gene expression.

Thus far, characterization and proof-of-concept demonstrations were accomplished using this CCCM system. Confirmation of the ability of the CCCM system to accurately mimic the in-vivo cardiac environment allows for the design and study of various conditions associated with CVD. These future studies are likely to include cardiomyocyte regeneration such as stem cells or adult cardiomyocyte regeneration using a mechanical loading environment, study of cardiac and aortic endothelial cells under conditions of continuous and pulsatile flow, ${ }^{114,115}$ and finally evaluation of drugs and other treatment options using cardiac cells maintained under various disease conditions.

\subsection{LIMITATIONS}

The CCCM system, however, has some limitations, including its complexity, the absence of an authentic 3D cell culture, and lack of electrical and hormonal factors. First of all, due to the complication of the system (tubes, valves, and multiple layers), it is time consuming to set up one experiment which includes the cleaning, setting, and operating processes. These can easily create a high risk of cross-contaminations. Second, ECM was constructed before seeding the cells; once seeded, the cells attach well on the surface

of the ECM. Generated tissues hence cannot be easily detached from the crosslinking 
collagen matrix, which can create a serious issue for tissue transplant or tissue regeneration. Thirdly, cardiomyocytes cultured in this system are in 2 dimensions on the top of the gel matrix, not inside the matrix, therefore lack of a 3D ECM model, which affects the development of the cardiac tissue including gene expression. Finally, beside physiological and mechanical aspects, in-vivo cardiomyocytes are influenced by electrical and hormonal stimulation. Mechanical, hormonal, and electrical aspects need to be incorporated simultaneously for cardiomyocyte culturing.

\subsection{FUTURE WORK}

The CCCM system opens a wide opportunity to understanding the behavior of cardiac cells on molecular and cellular levels. It also provides an excellent tool to regenerate mature cardiac tissue for tissue transplant, which is our ultimate goal. From the previous chapters, cardiac tissues cultured in the CCCM system become more mature via higher beat rate, more alignment, and stronger contraction,. However, in this study, cardiac cells were cultured on the surface of a soft collagen matrix and under mechanical stimulations of a normal heart condition. For tissue regeneration or tissue transplant, it is necessary to generate the 3D tissue without collagen matrix attached. For molecular and cellular studies, understanding the impact of mechanical stimulation for different cardiovascular conditions on a heart tissue is crucial to provide a better treatment options for CVD. In addition, with electrical pacing, the beating rate and the contraction increase significantly. This electrical pacing for the in-vitro cardiac tissue can be considered as the sinoatrial node in the heart, which generates impulses to the cardiac tissue at the greatest 
frequency. ${ }^{110}$ With these perspectives, the future works for this CCCM project might include:

\section{Using higher vertebrate (mammalian) tissues with 3D cell culture}

Embryonic chick cardiac cells have been commonly used in studies. However, tissues from higher mammal levels are more relevant to the human tissues. Also, cardiac tissue is more physiologically relevant to the in-vivo cardiac tissue if it is cultured within a 3D matrix. In addition, it is much better to use human iPS cell lines to study cardiac tissue behavior and regenerate tissues. Similar to the Flexcell system, 3D cell culture can be done in the cell culture chamber. In this case, the crosslinking matrix ring serves as an anchor for the cell to attach and keep the tissue from forming a ball. Primary testing results (not shown) proves that this technique can be used for cardiac culture under the mechanical stimulation in the CCCM system and warrants further investigation.

\section{Studies the effects of mechanical stimulations from various heart conditions}

Chapter 5 shows that this CCCM system is capable of replicating different pathological conditions. Molecular and cellular studies of cardiac tissue under pathological conditions can be specifically investigated.

\section{Create a co-existing electrical and mechanical stimulation within the system.}

Pilot studies with the CCCM system were conducted with simultaneous electrical and mechanical stimulation. The next step is to build a network for field electrical stimulation inside the cell chamber. When mechanical stimulations causing the tissue to go up and down, stretched and contracted, the electrical network must ensure that it won't create friction or tear the tissues apart. 


\section{REFERENCES}

1. Thom T, Haase N, Rosamond W, et al. Heart disease and stroke statistics - 2006 update - A report from the American Heart Association Statistics Committee and Stroke Statistics Subcommittee. Circulation 2006; 113(6): E85-E151.

2. Lloyd-Jones D, Adams RJ, Brown TM, et al. Heart Disease and Stroke Statistics2010 Update A Report From the American Heart Association. Circulation 2010; 121(7): E46-E215.

3. Go AS, Mozaffarian D, Roger VL, et al. Heart Disease and Stroke Statistics-2014 Update A Report From the American Heart Association. Circulation 2014; 129(3): E28E292.

4. Go AS, Mozaffarian D, Roger VL, et al. Heart Disease and Stroke Statistics-2013 Update A Report From the American Heart Association. Circulation 2013; 127(1): E6E245.

5. Website. Cardiovascular Disease Types. http://heartdiseaseemedtvcom/cardiovascular-disease/cardiovascular-disease-typeshtml.

6. Ezekowitz JA, Hernandez AF, Starling RC, et al. Standardizing care for acute decompensated heart failure in a large megatrial: The approach for the Acute Studies of Clinical Effectiveness of Nesiritide in Subjects with Decompensated Heart Failure (ASCEND-HF). American Heart Journal 2009; 157(2): 219-28.

7. Krum H, Abraham WT. Heart failure. Lancet 2009; 373(9667): 941-55.

8. McGrady M, Krum H. Screening: The New Frontier in Heart Failure Management. Cardiovascular Therapeutics 2009; 27(1): 1-3.

9. Newton PJ, Davidson PM, Krum H, Ollerton R, Macdonald P. Acute Hemodynamic Effects of Nebulised Furosemide in Stable Heart Failure. Journal of Cardiac Failure 2009; 15(6): S5-S.

10. Jessup M, Abraham WT, Casey DE, et al. 2009 Focused Update: ACCF/AHA Guidelines for the Diagnosis and Management of Heart Failure in Adults A Report of the American College of Cardiology Foundation/American Heart Association Task Force on Practice Guidelines. Circulation 2009; 119(14): 1977-2016. 
11. Hunt SA, Abraham WT, Chin MH, et al. 2009 Focused Update Incorporated Into the ACC/AHA 2005 Guidelines for the Diagnosis and Management of Heart Failure in Adults A Report of the American College of Cardiology Foundation/American Heart Association Task Force on Practice Guidelines. Circulation 2009; 119(14): E391-E479.

12. Frigerio M, Roubina E. Drugs for left ventricular remodeling in heart failure. American Journal of Cardiology 2005; 96(12A): 10L-8L.

13. Stevenson LW, Miller LW, Desvigne-Nickens P, et al. Left ventricular assist device as destination for patients undergoing intravenous inotropic therapy - A subset analysis from REMATCH (Randomized Evaluation of Mechanical Assistance in Treatment of Chronic Heart Failure). Circulation 2004; 110(8): 975-81.

14. Najam O, Yonan N, Williams SG, Shaw SM. The Usefulness of Chronic Heart Failure Treatments in Chronic Cardiac Graft Failure. Cardiovascular Therapeutics 2010; 28(1): 48-58.

15. Machida S, Matsuoka R, Noda S, et al. Evidence for the expression of neonatal skeletal myosin heavy chain in primary myocardium and cardiac conduction tissue in the developing chick heart. Developmental Dynamics 2000; 217(1): 37-49.

16. Tobita K, Liu LJ, Janczewski AM, et al. Engineered early embryonic cardiac tissue retains proliferative and contractile properties of developing embryonic myocardium. American Journal of Physiology-Heart and Circulatory Physiology 2006; 291(4): H1829-H37.

17. Schleich JM, Abdulla T, Summers R, Houyel L. An overview of cardiac morphogenesis. Archives of Cardiovascular Diseases 2013; 106(11): 612-23.

18. Manner J. Cardiac looping in the chick embryo: A morphological review with special reference to terminological and biomechanical aspects of the looping process. Anatomical Record 2000; 259(3): 248-62.

19. Goenezen S, Rennie MY, Rugonyi S. Biomechanics of early cardiac development. Biomechanics and Modeling in Mechanobiology 2012; 11(8): 1187-204.

20. Yelbuz TM, Waldo KL, Kumiski DH, et al. Shortened outflow tract leads to altered cardiac looping after neural crest ablation. Circulation 2002; 106(4): 504-10.

21. Guyton AC, editor. Textbook of Medical Physiology. 8th ed: W.B. Saunders Company, p.77-78; 1991.

22. Koeppen BM, Stanton B.A, editor. Berne \& Levy Physiology. Sixth ed: Mosby Elsevier, Section IV; 2008. 
23. Simmons CREaCA, editor. Introductory Biomechanics, From Cells to Organism: Cambridge University Press; 2007.

24. Klabunde RE. Ventricular Pressure-Volume Relationship. 2009. http://www.cvphysiology.com/Cardiac\%20Function/CF024.htm.

25. Asanoi H, Kameyama T, Ishizaka S, Nozawa T, Inoue H. Energetically optimal left ventricular pressure for the failing human heart. Circulation 1996; 93(1): 67-73.

26. Karki DB. Calculate left ventricular end diastolic pressure from Doppler Echocardiography. Kathmandu University Medical Journal 2006; 4, No.1(13): 139.

27. Olson EN, Brennan TJ, Chakraborty T, et al. Molecular Control of MyogenesisAntagonism Between Growth and Differentiation Molecular and Cellular Biochemistry 1991; 104(1-2): 7-13.

28. Mima T, Ueno H, Fischman DA, Williams LT, Mikawa T. Fibroblast Growth Factor Receptor is Required for In-Vivo Cardiac Myocyte Proliferation at Early Embryonic Stages of Heart Development. Proceedings of the National Academy of Sciences of the United States of America 1995; 92(2): 467-71.

29. Olson EN. Interplay Between Proliferation and Differentiaion Within the Myogenic Lineage. Developmental Biology 1992; 154(2): 261-72.

30. Eisenberg LM, Markwald RR. Cellular recruitment and the development of the myocardium. Developmental Biology 2004; 274(2): 225-32.

31. Hall CE, Hurtado R, Hewett KW, et al. Hemodynamic-dependent patterning of endothelin converting enzyme 1 expression and differentiation of impulse-conducting Purkinje fibers in the embryonic heart. Development 2004; 131(3): 581-92.

32. Sedmera D, Thompson RP. Myocyte Proliferation in the Developing Heart. Developmental Dynamics 2011; 240(6): 1322-34.

33. Chien KR, Knowlton KU, Zhu H, Chien S. Regulation of Cardiac GeneExpression During Myocaridal Growth and Hypotrophy-Molecular Studies of an Adpative Physiological Response. Faseb Journal 1991; 5(15): 3037-46.

34. Lee HR, Henderson SA, Reynolds R, Dunnmon P, Yuan D, Chien KR. Alpha-1Adrenergic Stimulation of Cardiac Gene Transscription in Neonatal Rat Myocardial Cells Effects on Myosin Light Chain Gene Exrpession. Journal of Biological Chemistry 1988; 263(15): 7352-8.

35. Sedmera D, Pexieder T, Vuillemin M, Thompson RP, Anderson RH. Developmental patterning of the myocardium. Anatomical Record 2000; 258(4): 319-37. 
36. Martin AF, Reddy MK, Zak R, Dowell RT, Rabinowi.M. Protein Metabolism in Hypertrophied Heart Muscle. Circulation Research 1974; 35(3): 32-42.

37. Zak R. Development and Proliferative Capacity of Cardiac Muscle Cells Circulation Research 1974; 35(2): 17-26.

38. Curtis MW, Russell B. Cardiac Tissue Engineering. Journal of Cardiovascular Nursing 2009; 24(2): 87-92.

39. Agarwal A, Farouz Y, Nesmith AP, Deravi LF, McCain ML, Parker KK. Micropatterning Alginate Substrates for In Vitro Cardiovascular Muscle on a Chip. Advanced Functional Materials 2013; 23(30): 3738-46.

40. Morgan HE, Gordon EE, Kira Y, et al. Biomechanical Mechanisms of Cardiac Hypertrophy. Annual Review of Physiology 1987; 49: 533-43.

41. Gregorio CC, Antin PB. To the heart of myofibril assembly. Trends in Cell Biology 2000; 10(9): 355-62.

42. Squire JM. Architecture and function in the muscle sarcomere. Current Opinion in Structural Biology 1997; 7(2): 247-57.

43. Sanger JW, Wang JS, Fan YL, White J, Sanger JM. Assembly and Dynamics of Myofibrils. Journal of Biomedicine and Biotechnology 2010.

44. Sanger JW, Kang SM, Siebrands CC, et al. How to build a myofibril. Journal of Muscle Research and Cell Motility 2005; 26(6-8): 343-54.

45. Ohtsuki I, Morimoto S, Takahashi-Yanaga F. Several aspects of calcium regulator mechanisms linked to troponin. In: Sugi H, ed. Molecular and Cellular Aspects of Muscle Contraction; 2003: 221-9.

46. Sanger JW, Ayoob JC, Chowrashi P, Zurawski D, Sanger JM. Assembly of myofibrils in cardiac muscle cells. In: Granzier HL, Pollack GH, eds. Elastic Filaments of the Cell; 2000: 89-105.

47. Klabunde RE. Signal Transduction 2009. http://www.cvphysiology.com/Blood\%20Pressure/BP011.htm.

48. Velez C, Aranega AE, Muros MA, et al. Quantification and distribution of troponin-T in cultures of chick-embryo moycardiocytes. . Acta Anatomica 1992; 145(3): 269-76.

49. Gomes AV, Guzman G, Zhao JJ, Potter JD. Cardiac troponin T Isoforms affect the $\mathrm{Ca} 2+$ sensitivity and inhibition of force development - Insights into the role of 
troponin T isoforms in the heart. Journal of Biological Chemistry 2002; 277(38): 353419.

50. Gomes AV, Potter JD, Szczesna-Cordary D. The role of troponins in muscle contraction. Iubmb Life 2002; 54(6): 323-33.

51. Adamcova M, Pelouch V. Isoforms of troponin in normal and diseased myocardium. Physiological Research 1999; 48(4): 235-47.

52. Crossley D, Altimiras J. Ontogeny of cholinergic and adrenergic cardiovascular regulation in the domestic chicken (Gallus gallus). American Journal of PhysiologyRegulatory Integrative and Comparative Physiology 2000; 279(3): R1091-R8.

53. Steinberg SF. The molecular basis for distinct beta-adrenergic receptor subtype actions in cardiomyocytes. Circulation Research 1999; 85(11): 1101-11.

54. Wallukat G. The beta-adrenergic receptors. Herz 2002; 27(7): 683-90.

55. Stewart DE, Kirby ML, Aronstam RS. Regulation of Beta-Adrenergic-receptor density in the noninnervated and denervated embryonic chick-heart. Journal of Molecular and Cellular Cardiology 1986; 18(5): 469-75.

56. Khan M, Mohsin S, Avitabile D, et al. Beta-Adrenergic Regulation of Cardiac Progenitor Cell Death Versus Survival and Proliferation. Circulation Research 2013; 112(3): 476-+.

57. Lompre AM. The sarco(endo)plasmic reticulum Ca2+-ATPases in the cardiovascular system during growth and proliferation. Trends in Cardiovascular Medicine 1998; 8(2): 75-82.

58. Kang L, Fang Q, Hu SJ. Regulation of phospholamban and sarcoplasmic reticulum $\mathrm{Ca} 2+$ ATPase by atorvastatin: Implication for cardiac hypertrophy. Archives of Pharmacal Research 2007; 30(5): 596-602.

59. Klabunde RE. Cardiac Moycytes and Sacromeres. 2009. http://www.cvphysiology.com/Cardiac\%20Function/CF020.htm.

60. Balnave CD, Davey DF, Allen DG. Distribution of sarcomere length and intracellular calcium in mouse skeletal muscle following stretch-induced injury. Journal of Physiology-London 1997; 502(3): 649-59.

61. Nishimura S, Yamashita H, Katoh $\mathrm{M}$, et al. Contractile dysfunction of cardiomyopathic hamster myocytes is pronounced under high load conditions. Journal of Molecular and Cellular Cardiology 2005; 39(2): 231-9. 
62. Klabunde RE. Cardiac Excitation-Contraction Coupling. 2009. http://www.cvphysiology.com/Cardiac\%20Function/CF022.htm.

63. Klabunde RE. Ventricular Pressure-Volume Relationship. Cardiovascular Therapeutics 2009.

64. Pandya K, Pulli B, Bultman S, Smithies O. Reversible Epigenetic Modifications of the Two Cardiac Myosin Heavy Chain Genes During Changes in Expression. Gene Expression 2011; 15(2): 51-9.

65. Mamidi R, Chandra M. Divergent effects of alpha- and beta-myosin heavy chain isoforms on the $\mathrm{N}$ terminus of rat cardiac troponin T. Journal of General Physiology 2013; 142(4): 413-23.

66. James J, Hor K, Moga MA, Martin LA, Robbins J. Effects of myosin heavy chain manipulation in experimental heart failure. Journal of Molecular and Cellular Cardiology 2010; 48(5): 999-1006.

67. Morkin E. Control of cardiac myosin heavy chain gene expression. Microscopy Research and Technique 2000; 50(6): 522-31.

68. $\mathrm{Yu} \mathrm{JG}$, Russell B. Cardiomyocyte remodeling and sarcomere addition after uniaxial static strain in vitro. Journal of Histochemistry \& Cytochemistry 2005; 53(7): $839-44$.

69. Samarel AM. Costameres, focal adhesions, and cardiomyocyte mechanotransduction. American Journal of Physiology-Heart and Circulatory Physiology 2005; 289(6): H2291-H301.

70. Kaneko T, Kojima K, Yasuda K. Dependence of the community effect of cultured cardiomyocytes on the cell network pattern. Biochemical and Biophysical Research Communications 2007; 356(2): 494-8.

71. de Bivort B, Huang S, Bar-Yam Y. Dynamics of cellular level function and regulation derived from murine expression array data. Proceedings of the National Academy of Sciences of the United States of America 2004; 101(51): 17687-92.

72. Maron BJ. Hypertrophic cardiomyopathy. Circulation 2002; 106(19): 2419-21.

73. Curl CL, Delbridge LMD, Canny BJ, Wendt IR. Testosterone Modulates Cardiomyocyte Ca2+ Handling and Contractile Function. Physiological Research 2009; 58(2): 293-7.

74. Yeih DF, Lin LY, Yeh HI, et al. Temporal changes in cardiac force- and flowgeneration capacity, loading conditions, and mechanical efficiency in streptozotocin- 
induced diabetic rats. American Journal of Physiology-Heart and Circulatory Physiology 2008; 294(2): H867-H74.

75. Gazmuri RJ, Berkowitz M, Cajigas H. Myocardial effects of ventricular fibrillation in the isolated rat heart. Critical Care Medicine 1999; 27(8): 1542-50.

76. Kummar S, Rubinstein L, Kinders R, et al. Phase 0 clinical trials: Conceptions and misconceptions. Cancer Journal 2008; 14(3): 133-7.

77. Marchetti S, Schellens JHM. The impact of FDA and EMEA guidelines on drug development in relation to Phase 0 trials. British Journal of Cancer 2007; 97(5): 577-81.

78. Tanaka Y, Morishima K, Shimizu T, et al. Demonstration of a PDMS-based biomicroactuator using cultured cardiomyocytes to drive polymer micropillars. Lab on a Chip 2006; 6(2): 230-5.

79. Al Naieb S, Happel CM, Yelbuz TM. A detailed atlas of chick heart development in vivo. Annals of Anatomy-Anatomischer Anzeiger 2013; 195(4): 324-41.

80. Keller BB. New insights into the developmental biomechanics of the atrioventricular valves. Circulation Research 2007; 100(10): 1399-401.

81. Lucitti JL, Visconti R, Novak J, Keller BB. Increased arterial load alters aortic structural and functional properties during embryogenesis. American Journal of Physiology-Heart and Circulatory Physiology 2006; 291(4): H1919-H26.

82. Keller BB, Liu LJ, Tinney JP, Tobita K. Cardiovascular developmental insights from embryos. In: Elad D, Young RC, eds. Reproductive Biomechanics; 2007: 377-88.

83. DeSantiago J, Maier LS, Bers DM. Frequency-dependent acceleration of relaxation in the heart depends on CaMKII, but not phospholamban. Journal of Molecular and Cellular Cardiology 2002; 34(8): 975-84.

84. Filas BA, Bayly PV, Taber LA. Mechanical Stress as a Regulator of Cytoskeletal Contractility and Nuclear Shape in Embryonic Epithelia. Annals of Biomedical Engineering 2011; 39(1): 443-54.

85. Wollert KC, Drexler H. Clinical applications of stem cells for the heart. Circulation Research 2005; 96(2): 151-63.

86. Reinlib L, Field L. Cell transplantation as future therapy for cardiovascular disease? A workshop of the National Heart, Lung, and Blood Institute. Circulation 2000; 101(18): E182-E7.

87. Fujimoto KL, Tobita K, Merryman WD, et al. An elastic, biodegradable cardiac patch induces contractile smooth muscle and improves cardiac remodeling and function 
in subacute myocardial infarction. Journal of the American College of Cardiology 2007; 49(23): 2292-300.

88. Galvez-Monton C, Prat-Vidal C, Roura S, Soler-Botija C, Bayes-Genis A. Cardiac Tissue Engineering and the Bioartificial Heart. Revista Espanola De Cardiologia 2013; 66(5): 391-9.

89. Yang ZJ, Shen WZ, Rottman JN, Murray K. Rapid stimulation causes electrical remodeling in cultured atrial cells. Biophysical Journal 2003; 84(2): 425A-A.

90. Parker KK, Tan J, Chen CS, Tung L. Myofibrillar architecture in engineered cardiac myocytes. Circulation Research 2008; 103(4): 340-2.

91. Kemi OJ, Arbo I, Hoydal MA, et al. Reduced $\mathrm{pH}$ and contractility in failing rat cardiomyocytes. Acta Physiologica 2006; 188(3-4): 185-93.

92. Evans HJ, Sweet JK, Price RL, Yost M, Goodwin RL. Novel 3D culture system for study of cardiac myocyte development. American Journal of Physiology-Heart and Circulatory Physiology 2003; 285(2): H570-H8.

93. Khait L, Birla RK. Changes in Gene Expression During the Formation of Bioengineered Heart Muscle. Artificial Organs 2009; 33(1): 3-15.

94. Zimmermann WH, Schneiderbanger K, Schubert P, et al. Tissue engineering of a differentiated cardiac muscle construct. Circulation Research 2002; 90(2): 223-30.

95. Zimmermann WH, Fink C, Kralisch D, Remmers U, Weil J, Eschenhagen T. Three-dimensional engineered heart tissue from neonatal rat cardiac myocytes. Biotechnology and Bioengineering 2000; 68(1): 106-14.

96. Tanaka Y, Morishima K, Shimizu T, et al. An actuated pump on-chip powered by cultured cardiomyocytes. Lab on a Chip 2006; 6(3): 362-8.

97. Khademhosseini A, Langer R, Borenstein J, Vacanti JP. Microscale technologies for tissue engineering and biology. Proceedings of the National Academy of Sciences of the United States of America 2006; 103(8): 2480-7.

98. Griffith LG, Swartz MA. Capturing complex 3D tissue physiology in vitro. Nature Reviews Molecular Cell Biology 2006; 7(3): 211-24.

99. Geckil $\mathrm{H}, \mathrm{Xu} \mathrm{F}$, Zhang $\mathrm{XH}$, Moon S, Demirci U. Engineering hydrogels as extracellular matrix mimics. Nanomedicine 2010; 5(3): 469-84.

100. Dahlmann J, Krause A, Moller L, et al. Fully defined in situ cross-linkable alginate and hyaluronic acid hydrogels for myocardial tissue engineering. Biomaterials 2013; 34(4): 940-51. 
101. Bursac N, Parker KK, Iravanian S, Tung L. Cardiomyocyte cultures with controlled macroscopic anisotropy - A model for functional electrophysiological studies of cardiac muscle. Circulation Research 2002; 91(12): E45-E54.

102. Camelliti P, McCulloch AD, Kohl P. Microstructured cocultures of cardiac myocytes and fibroblasts: A two-dimensional in vitro model of cardiac tissue. Microscopy and Microanalysis 2005; 11(3): 249-59.

103. McDevitt TC, Angello JC, Whitney ML, et al. In vitro generation of differentiated cardiac myofibers on micropatterned laminin surfaces. Journal of Biomedical Materials Research 2002; 60(3): 472-9.

104. Park J, Kim IC, Cha JM, Park S, Lee J, Kim B. Mechanotransduction of cardiomyocytes interacting with a thin membrane transducer. Journal of Micromechanics and Microengineering 2007; 17(6): 1162-7.

105. Feinberg AW, Feigel A, Shevkoplyas SS, Sheehy S, Whitesides GM, Parker KK. Muscular thin films for building actuators and powering devices. Science 2007; 317(5843): 1366-70.

106. Gwak SJ, Bhang SH, Kim IK, et al. The effect of cyclic strain on embryonic stem cell-derived cardiomyocytes. Biomaterials 2008; 29(7): 844-56.

107. Gupta V, Grande-Allen KJ. Effects of static and cyclic loading in regulating extracellular matrix synthesis by cardiovascular cells. Cardiovascular Research 2006; 72(3): 375-83.

108. Park JY, Kim IC, Baek JG, et al. Micro pumping with cardiomyocyte-polymer hybrid. Lab on a Chip 2007; 7(10): 1367-70.

109. Burstein B, Qi XY, Yeh YH, Calderone A, Nattel S. Atrial cardiomyocyte tachycardia alters cardiac fibroblast function: A novel consideration in atrial remodeling. Cardiovascular Research 2007; 76(3): 442-52.

110. Koeppen BM, Stanton B.A Berne \& Levy Physiology. Mosby Elservier, Section IV 2008, Sixth Ed.: chapter 13 and 6.

111. Nguyen MD, Giridharan GA, Estrada R, et al. Microfluidic Cardiac Cell Culture Model (mu CCCM). Analytical Chemistry 2010; 82(18): 7581-7.

112. Simmerman HKB, Jones LR. Phospholamban: Protein structure, mechanism of action, and role in cardiac function. Physiological Reviews 1998; 78(4): 921-47.

113. MacLennan DH, Kranias EG. Phospholamban: A crucial regulator of cardiac contractility. Nature Reviews Molecular Cell Biology 2003; 4(7): 566-77. 
114. Estrada R, Giridharan GA, Nguyen MD, Prabhu SD, Sethu P. Microfluidic endothelial cell culture model to replicate disturbed flow conditions seen in atherosclerosis susceptible regions. Biomicrofluidics 2011; 5(3).

115. Estrada R, Giridharan GA, Nguyen MD, et al. Endothelial Cell Culture Model for Replication of Physiological Profiles of Pressure, Flow, Stretch, and Shear Stress in Vitro. Analytical Chemistry 2011; 83(8): 3170-7.

116. Klauke N, Smith G, Cooper JM. Local Regional Stimulation of Single Isolated Ventricular Myocytes Using Microfluidics. Analytical Chemistry 2009; 81(15): 6390-8.

117. Inya-Agha O, Klauke N, Davies T, Smith G, Cooper JM. Spectroscopic probing of dynamic changes during stimulation and cell remodeling in the single cardiac myocyte. Analytical Chemistry 2007; 79(12): 4581-7.

118. Klauke N, Smith GL, Cooper JM. Microfluidic partitioning of the extracellular space around single cardiac myocytes. Analytical Chemistry 2007; 79(3): 1205-12.

119. Yin HB, Zhang XL, Pattrick N, et al. Influence of hydrodynamic conditions on quantitative cellular assays in microfluidic systems. Analytical Chemistry 2007; 79(18): 7139-44.

120. Li XJ, Li PCH. Microfluidic selection and retention of a single cardiac myocyte, on-chip dye loading, cell contraction by chemical stimulation, and quantitative fluorescent analysis of intracellular calcium. Analytical Chemistry 2005; 77(14): 431522.

121. Tanaka Y, Sato K, Shimizu T, Yamato M, Okano T, Kitamori T. A microspherical heart pump powered by cultured cardiomyocytes. Lab on a Chip 2007; 7(2): 207-12.

122. Nguyen MD, Tinney JP, Yuan FP, et al. Cardiac Cell Culture Model As a Left Ventricle Mimic for Cardiac Tissue Generation. Analytical Chemistry 2013; 85(18): 8773-9.

123. Lotters JC, Olthuis W, Veltink PH, Bergveld P. The mechanical properties of the rubber elastic polymer polydimethylsiloxane for sensor applications. Journal of Micromechanics and Microengineering 1997; 7(3): 145-7.

124. Schneider F, Fellner T, Wilde J, Wallrabe U. Mechanical properties of silicones for MEMS. Journal of Micromechanics and Microengineering 2008; 18(6).

125. Mata A, Fleischman AJ, Roy S. Characterization of polydimethylsiloxane (PDMS) properties for biomedical micro/nanosystems. Biomedical Microdevices 2005; 7(4): 281-93. 
126. Liu M, Chen QF. Characterization study of bonded and unbonded polydimethylsiloxane aimed for bio-micro-electromechanical systems-related applications. Journal of Micro-Nanolithography Mems and Moems 2007; 6(2).

127. Khanafer K, Duprey A, Schlicht M, Berguer R. Effects of strain rate, mixing ratio, and stress-strain definition on the mechanical behavior of the polydimethylsiloxane (PDMS) material as related to its biological applications. Biomedical Microdevices 2009; 11(2): 503-8.

128. Liu M, Sun JR, Sun Y, Bock C, Chen QF. Thickness-dependent mechanical properties of polydimethylsiloxane membranes. Journal of Micromechanics and Microengineering 2009; 19(3).

129. Taber LA, Sun H, Clark EB, Keller BB. Epicardial strains in embryonic chick ventricle at stage-16 through stage-24. . Circulation Research 1994; 75(5): 896-903.

130. Tobita K, Keller BB. Right and left ventricular wall deformation patterns in normal and left heart hypoplasia chick embryos. American Journal of Physiology-Heart and Circulatory Physiology 2000; 279(3): H959-H69.

131. White FM, editor. Fluid Mechanics: McGraw-Hill Companies, Inc. Pg. 25-26; 2008.

132. Forte G, Pagliari S, Ebara M, et al. Substrate Stiffness Modulates Gene Expression and Phenotype in Neonatal Cardiomyocytes In Vitro. Tissue Engineering Part A 2012; 18(17-18): 1837-48.

133. Pagliari S, Forte G, Ebara M, et al. Substrate stiffness modulates phenotype and gene expression in neonatal cardiomyocytes in vitro. J Tissue Eng Regen Med 2012; 6: 239-.

134. Cimetta E, Godier-Furnemont A, Vunjak-Novakovic G. Bioengineering heart tissue for in vitro testing. Current Opinion in Biotechnology 2013; 24(5): 926-32.

135. Dunn DA, Hodge AJ, Lipke EA. Biomimetic materials design for cardiac tissue regeneration. Wiley Interdisciplinary Reviews-Nanomedicine and Nanobiotechnology 2014; 6(1): 15-39.

136. Prabhakaran MP, Venugopal J, Kai D, Ramakrishna S. Biomimetic material strategies for cardiac tissue engineering. Materials Science \& Engineering C-Materials for Biological Applications 2011; 31(3): 503-13.

137. Silvestri A, Boffito M, Sartori S, Ciardelli G. Biomimetic Materials and Scaffolds for Myocardial Tissue Regeneration. Macromolecular Bioscience 2013; 13(8): 984-1019. 
138. Hazeltine L, Simmons C, Salick M, Crone W, Pruitt B, Palecek S. Effects of substrate mechanics on yield and contractility of cardiomyocytes generated from pluripotent stem cells. Abstracts of Papers of the American Chemical Society 2011; 241.

139. Bajaj P, Tang X, Saif TA, Bashir R. Stiffness of the substrate influences the phenotype of embryonic chicken cardiac myocytes. Journal of Biomedical Materials Research Part A 2010; 95A(4): 1261-9.

140. Clause KC, Tinney JP, Liu LJ, Keller BB, Tobita K. Engineered Early Embryonic Cardiac Tissue Increases Cardiomyocyte Proliferation by Cyclic Mechanical Stretch via p38-MAP Kinase Phosphorylation. Tissue Engineering Part A 2009; 15(6): 1373-80.

141. Damink L, Dijkstra PJ, Vanluyn MJA, Vanwachem PB, Nieuwenhuis P, Feijen J. Glutaraldyhdye as a cross-linking agent for collagen-based biomaterials. . Journal of Materials Science-Materials in Medicine 1995; 6(8): 460-72.

142. Rault I, Frei V, Herbage D, AbdulMalak N, Huc A. Evaluation of different chemical methods for cross-linking collagen gel, films and sponges. Journal of Materials Science-Materials in Medicine 1996; 7(4): 215-21.

143. Croissant JD, Carpenter S, Bader D. Identification and genomic cloning of $\mathrm{CMHC1}$ - A unique myosin heavy chain expressed exclusively in the developing chicken heart. Journal of Biological Chemistry 2000; 275(3): 1944-51.

144. Martinsen BJ. Reference guide to the stages of chick heart embryology. Developmental Dynamics 2005; 233(4): 1217-37.

145. Russell B, Curtis MW, Koshman YE, Samarel AM. Mechanical stress-induced sarcomere assembly for cardiac muscle growth in length and width. Journal of Molecular and Cellular Cardiology 2010; 48(5): 817-23.

146. Bonet J, R. D. Wood, R. D. Nonlinear continuum mechanics for finite element analysis: Cambridge University Press; 1997.

147. Remondino A, Kwon SH, Communal C, et al. beta-adrenergic receptor-stimulated apoptosis in cardiac myocytes is mediated by reactive oxygen species/c-Jun NH2terminal kinase-dependent activation of the mitochondrial pathway. Circulation Research 2003; 92(2): 136-8.

148. Ostadal B, Janatova T, Krause EG, Rychter Z, Pelouch V. Analysis of the Cardiotoxic Effect of Isoproterenol in Chick-Embryo. Journal De Physiologie 1983; 78(9): 867-9.

149. Ostadal B, Pelouch V, Ostadalova I, Novakova O. Structural and Biochemical Remodeling in Catecholamine-Induced Cardiomyopathy-Comparative and Ontogenic Aspects. . Molecular and Cellular Biochemistry 1995; 147(1-2): 83-8. 
150. Higgins D, Pappano AJ. Developmental-changes in the sensitivity of the chickembryo ventricle to Beta-adrenergic agonist during adrenergic-innervation. Circulation Research 1981; 48(2): 245-53.

151. Severin E, Sartore S, Schiaffino S. Direct toxic effect of isoproterenol on cultured cardiac-muscle cells. Experientia 1977; 33(11): 1489-.

152. Inesi G, Prasad AM, Pilankatta R. The Ca2+ ATPase of cardiac sarcoplasmic reticulum: Physiological role and relevance to diseases. Biochemical and Biophysical Research Communications 2008; 369(1): 182-7.

153. Sjoblom B, Salmazo A, Djinovic-Carugo K. alpha-actinin structure and regulation. Cellular and Molecular Life Sciences 2008; 65(17): 2688-701.

154. Mills MA, Yang N, Weinberger RP, et al. Differential expression of the actinbinding proteins, alpha-actinin-2 and-3, in different species: implications for the evolution of functional redundancy. Human Molecular Genetics 2001; 10(13): 1335-46.

155. Lompre AM, Anger M, Levitsky D. Sarco(endo)plasmic reticulum calcium pumps in the cardiovascular-system-function and gene-expession. . Journal of Molecular and Cellular Cardiology 1994; 26(9): 1109-21.

156. Krenz M, Robbins J. Impact of beta-myosin heavy chain expression on cardiac function during stress. Journal of the American College of Cardiology 2004; 44(12): 2390-7. 


\section{APPENDIX}

\section{LIST OF ABBREVIATIONS}

$\begin{array}{ll}\mathrm{A}_{o} & \text { Original cross-sectional area } \\ \mathrm{AC} & \text { Adenyl cyclic } \\ \mathrm{AR} & \text { Adrenergic Receptor } \\ \mathrm{ATP} & \text { Adenosine triphosphate } \\ \mathrm{BSA} & \text { Bovine Serum Albumin } \\ \mathrm{BrdU} & \text { Bromodeoxyuridine } \\ \mathrm{BR} & \text { Beat rate } \\ \mathrm{cAMP} & \text { cyclic Adenosine Monophosphate } \\ \mathrm{Ca} & \text { Calcium ion } \\ \mathrm{CB} & \text { Cork Borer } \\ \mathrm{CCCM} & \text { Cardiomyocyte Cell Culture Model } \\ \mathrm{CM} & \text { Cardiomyocytes } \\ \mathrm{CNC} & \text { Computer Numerical Control } \\ \mathrm{cm} & \text { centimeter } \\ \mathrm{CVD} & \text { Cardiovascular disease } \\ \mathrm{d} & \text { Diameter } \\ \mathrm{DI} \text { water } & \text { De-ionized water }\end{array}$




\begin{tabular}{|c|c|}
\hline DMEM & Dulbecco's Modified Eagles's Medium \\
\hline $\mathrm{E}$ & Young's Modulus \\
\hline ECM & Extra-cellular matrix \\
\hline $\mathrm{F}$ & Tensile force \\
\hline FBS & Fetal Bovine Serum \\
\hline FEA & Finite Element Analysis \\
\hline $\mathrm{h}$ & Height \\
\hline $\mathrm{HF}$ & Heart Failure \\
\hline $\mathrm{HH}$ & Hamburger Hamilton \\
\hline ID & Inner diameter \\
\hline ISO & Isoproterenol \\
\hline $1, \mathrm{~L}$ & Length \\
\hline $\mathrm{L}_{\mathrm{o}}$ & Original length of the membrane \\
\hline LV & Left ventricle \\
\hline LVAD & Left ventricle assistant device \\
\hline $\mathrm{L}_{0}$ & Length between two black dots at $0.0 \mathrm{mmHg}$ \\
\hline $\mathrm{L}_{\mathrm{f}}$ & Length between two black dots after pressure is appliec \\
\hline MHC & Myosin Heavy Chain \\
\hline $\mathrm{mL}$ & Milliliter \\
\hline $\mathrm{mm}$ & Millimeter \\
\hline $\mathrm{N}$ & Total number of the sample \\
\hline OD & Outer diameter \\
\hline PBS & Phosphate Buffer Saline \\
\hline
\end{tabular}




\begin{tabular}{|c|c|}
\hline PDMS & Polydimethyl siloxane \\
\hline PFA & Paraformaladehyde \\
\hline PKA & Protein Kinase A \\
\hline PLB, PLN & Phospholamban \\
\hline pPLB, pPLN & Phosphorylated phospholamban \\
\hline $\mathrm{R}$ & Radius \\
\hline $\mathrm{R}_{\mathrm{f}}$ & Final radius \\
\hline $\mathrm{R}_{\mathrm{i}}$ & Initial radius \\
\hline RIE & Reactive Ion Etcher \\
\hline rpm & revolution per minute \\
\hline RT & Room Temperature \\
\hline SERCA2a & Sarcoplasmic reticulum calcium ATPase 2a \\
\hline SR & Sarcoplasmic reticulum \\
\hline $\operatorname{TnT}$ & Troponin $\mathrm{T}$ \\
\hline V & Volume \\
\hline$\sigma$ & Engineering stress \\
\hline$\sigma^{*}$ & True stress \\
\hline$\varepsilon$ & Engineering strain \\
\hline$\varepsilon^{*}$ & True strain \\
\hline$\varepsilon_{\mathrm{r}}$ & Radial strain \\
\hline$\varepsilon_{\mathrm{c}}$ & Circumferential strain \\
\hline$\lambda_{\mathrm{c}}$ & Circumferential stretch ratio \\
\hline
\end{tabular}




\section{Shear stress}

$\mu$

Viscosity

$\mu \mathrm{CCCM}$

Microfluidic cardiac cell culture model

$\mu \mathrm{L}$

Microliter 


\section{CURICULLUM VITAE}

Education:

08/2010- present

08/2008- 08/2010

08/1995- 08/1999

\author{
MAI-DUNG THI NGUYEN \\ dmnguy01@gmail.com
}

\section{The University of Louisville, KY}

Pursuing a PhD in Mechanical Engineering focusing in Biomedical Research

Project: The Second Generation of CCCM System for In-Vitro

Cardiac Tissue Engineering

Month for graduation: 08/2014

\section{The University of Louisville, KY}

MS in Mechanical Engineering/Biomedical Research

The University of Oklahoma, OK

BS in Chemical Engineering/ Biotechnology Option

\section{Professional Experience:}

\section{Graduate Research Assistant}

Mentor and co-mentors: Dr. G. Giridhan, Dr. P. Sethu, and Dr.

B. Keller

+ Bioengineering Engineering Department, the University of Louisville, KY

+ Pediatric Cardiac Research Department, the University of Louisville, KY

+ Design, fabricate, validate and operate systems for cardiovascular research.

+ Mentor and train middle school, high school, undergraduate and new coming graduate students.

08/2005- 05/2007 Research Technologist II

Supervisor: Dr. B. Clark

Biochemistry \& Molecular Biology Department, the University of Louisville, KY

Study the effects of $B t_{2} c A M P$ on the expression of COUP-TF, $S T A R$, and $S F-1$ in diabetic diseases. 
08/2004- 08/2005

Research Technologist II

Supervisors: Dr. P. Feldhoff and Dr. H. Lassiter.

Pediatrics and Neonatology Department, the University of

Louisville, KY

Study the regulation of $C_{3}$ and $C_{9}$ in stroked infant rat brain

11/1999- 06/2003 Research Assistant

Supervisor: Dr. L. Griffith

Department of Neuroscience and Biochemistry, Brandeis

University, MA

Study the effect of eag Potassium Channel on Calcium/

Calmodulin Dependent Protein Kinase II

\section{Publications in Journals:}

- Mai-Dung Nguyen, Joseph P. Tinney, et.al; Cardiac Cell Culture Model as a Left Ventricle Mimic for Cardiac Tissue Generation; Anal. Chem., August 16, 2013.

- Rosendo Estrada, Guruprasad A. Giridharan, Mai-Dung Nguyen, et.al, Endothelial Cell Culture Model for Replication of Physiological Profiles of Pressure, Flow, Stretch, and Shear Stress in Vitro, Anal. Chem., April 15 2011, 83 (8), pp 3170-7

- $\quad$ Rosendo Estrada, Guruprasad Giridharan, Mai-Dung Nguyen, et.al. "Microfluidic Endothelial Cell Culture Model to Replicate Disturbed Flow Conditions Seen in Atherosclerosis Susceptible Regions” Biomicrofluidics, 5, 032006, 2011

- Mai-Dung Nguyen, Guruprasad Giridharan, Rosendo Estrada, et al, “Microfluidic Cardiac Cell Culture Model ( $\mu$ CCCM)” Anal. Chem., 2010, 82 (18), pp 7581-7587.

- William N. White, Ashok Raj, Mai-Dung Nguyen, et al, "Clinical application of microfluidic leukocyte enrichment protocol in mild phenotype sickle cell disease (SCD).” Biomed Microdevices (2009) 11:477-483

- Lori A. Devlin, Mai-Dung Nguyen, et. al. "Effects of endotoxin administration and cerebral hypoxia-ischemia on complement activity and local transcriptional regulation in neonatal rats." Neuroscience Letters, Vol. 390, Issue 2, December 23, 2005, p. 109-113. 
- Xiu Xia Sun, James J.L Hodge,Yi Zhou, Mai-Dung Nguyen, and Leslie C. Griffith., "The eag Potassium Channel Binds and Locally Activates Calcium/ Calmodulin Dependent Protein Kinase II." The Journal of Biological Chemistry, Vol. 279, No. 11, March 12, 2004, p. 10206-10214.

\section{Selected Conference Proceedings:}

- Mai-Dung Nguyen,Joseph P. Tinney, Fangping Yuan, Thomas Roussel, Guruprasad Giridharan, Bradley B. Keller, and Palaniappan Sethu, "Significant Mechanical and electrical stimulating effects on generation of $3 D$ cardiac tissue via CCCM system.”, Oral Presentation, September 27, BMES 2013, Seattle, WA

- Mai-Dung Nguyen,Joseph P. Tinney, Fangping Yuan, Thomas Roussel, Guruprasad Giridharan, Bradley B. Keller, and Palaniappan Sethu, "An In-Vitro Model System for Generation of Mechanically Conditioned Cardiac Tissue Constructs”, Poster Presentation, April 12, MMB 2013, Marina De Rey, CA.

- Mai-Dung Nguyen, Joseph P. Tinney, Fangping Yuan, Bradley B. Keller, Guruprasad Giridharan, and Palaniappan Sethu, "Cardiac Cell Culture Model for Cardiac Tissue Generation." Oral Presentation, Oct. 25 ${ }^{\text {th }}$, BMES 2012, Atlanta, GA

- Mai-Dung Nguyen, Kranthi K. Bhavanam, Awdhesh Kalia, Yongsheng Lian, and Palaniappan Sethu, "Microfluidic Cell Arrays for High-Throughput Culture." Poster Presentation, 2011 May $26^{\text {th }}$, 7th KIEC and 16th KY EPSCoR-Joint Conference, Louisville, KY

- Mai-Dung Nguyen, Guruprasad Giridharan, Rosendo Estrada, Sumanth D. Prabhu, and Palaniappan Sethu, "Cardiac Cell Culture Model to Study Cardiomyocytes under Physiological Loads. ” Poster Presentation, BMES 2010, October - The 2010 Annual Meeting of the Biomedical Engineering Society, Austin, TX

- Rosendo Estrada, Vahid Parichehreh, Mai-Dung Nguyen, and Palaniappan Sethu, "Microfluidic Devices to Study the Effect of Atherogenic Flow Pattern on Cultured Endothelial Cells." Poster Presentation, BMEMS 2010, October - The 2010 Annual Meeting of the Biomedical Engineering Society, Austin, TX

- Mai-Dung Nguyen, Guruprasad Giridharan, Sumanth D. Prabhu and Palaniappan Sethu, "Microfluidic Cardiac Circulation Model for Functional 
Cardiomyocyte Studies”, Conf Proc IEEE Eng Med Biol Soc. 2009;1:1060-3, Minneapolis, MN

- Mai-Dung Nguyen, Guruprasad Giridharan, Palaniappan Sethu, "Cardiomyocyte Acutuator for Microfluidics Platform Nozzle-Diffuser Pump." Poster presentation, MicroTAS 2008, October- Twelfth International Conference on Miniaturized System for Chemistry and Life Science, San Diego, CA.

- William White, Mai-Dung Nguyen, and Palaniappan Sethu, "Microfluidics enumeration protocol for comprehensive profiling of nucleated cells in mild phenotype sickle cells disease (SCD)." Poster presentation, MicroTAS 2008, October- Twelfth International Conference on Miniaturized System for Chemistry and Life Science, San Diego, CA.

- Bobby L. Gibbons, Mai-Dung Nguyen, Guruprasad Giridharan, Mohammed Ishmahil, Sumant D. Prabhu and Palaniappan Sethu, "Microfluidic Platform to Study Cardiomyocyte Mechanotransduction", Research Louisville! 2008, Louisville, KY. (2 ${ }^{\text {nd }}$ Place, Interdisciplinary Research Category)

\section{Invention Disclosures:}

Palaniappan Sethu, Guruprasad Giridharan, Rosendo Estrada, Mai-Dung Nguyen and Sumanth D. Prabhu, "Cardiac and Endothelial Cell Culture Models and Methods."

\section{Honors and Affiliations:}

2010-present Southern Regional Education Board (SREB) Doctoral Fellow Scholarship 2010-present Member of Biomedical Engineering Society 2009-present Member of the Society of Engineering Women

2008

1999

Best Poster Award Team, University of Louisville, Engineering Expo Second Team Work Award with the Senior Design Project of Energy Retrofit

1998 Best Award for Laboratory Chemical Engineering Advanced Design

\section{Other Achievements and Professional Conference Attendances:}

$05 / 2013$

$10 / 2013$

$10 / 2011$

$10 / 2010$
Receive a certificate for Graduate Teaching Academy. Attend "Institute on Teaching and Mentoring: Faculty Diversity", Arlington, VA.

Attend "Institute on Teaching and Mentoring: Faculty Diversity", Atlanta, GA. Attend "Institute on Teaching and Mentoring: Faculty Diversity", Tampa, FL. 


\section{Outreach Activities:}

10/2005-present St. JOHN VIANNEY CHURCH, KY

Teach Sunday Catechism

$4 / 2 / 2011$

LOUISVILLE SCIENCE CENTER, KY

Participate the demo "Heart on a chip"

Bioengineering Activities for Nanodays.

3/1/2011 UNIVERSITY of LOUISVILLE, KY

Participate in the demo "Cardiovascular Cell Culture Model"

Engineering Career Day

01/2007-11/2007 BAPTIST EAST HOSPITAL, KY

Participate in Pastoral Care Ministry

10/1999-06/2003 St. WILLIAM CHURCH, MA

Teach Sunday Catechism

\section{References:}

Provided upon request 